\title{
A Guidance and Control Assessment of Three Vertical Landing Options for RLV
}

M. Gallaher, D. Coughlin, and D. Krupp

Marshall Space Flight Center - MSFC, Alabama 



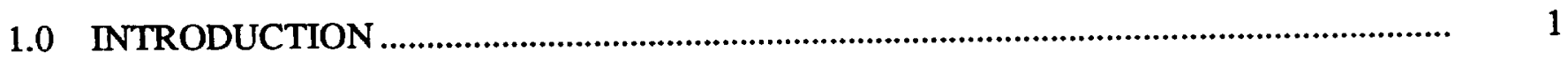

1.1 Purpose.......................................................................................................................

1.2 Landing Concepts ...................................................................................................

1.2.1 Aerodynamic Inversion Maneuver ............................................................................ 2

1.2.2 Propulsive Inversion Maneuver ............................................................................. 2

1.2.3 Powered Pullup Maneuver.................................................................................. 2

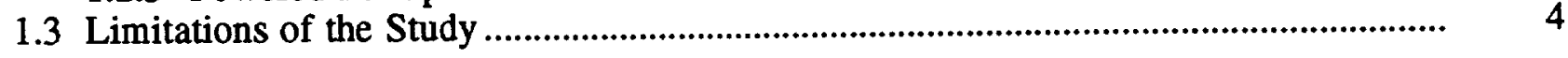

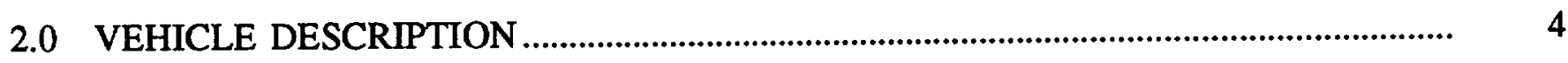

2.1 Mass Properties....................................................................................................... 4

2.2 Aerodynamic Data..................................................................................................

2.3 Main Engines.............................................................................................................. 7

2.4 Thrust Vector Control Actuators........................................................................ 7

2.5 Reaction Control System Thrusters ............................................................................

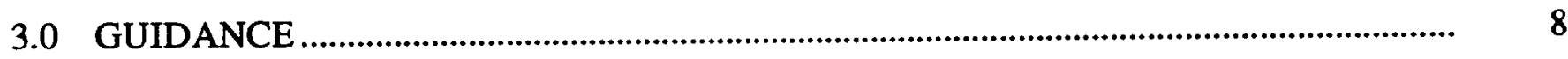

3.1 Introduction ............................................................................................................... 8

3.2 Development........................................................................................................... 9

3.2.1 Thrust Acceleration Profile ..................................................................................... 9

3.2.2 Selection of $p_{i}(t)$ Functions............................................................................. 11

3.3 Guidance Discussion..................................................................................................... 12

3.3.1 Aerodynamic Accelerations............................................................................... 12

3.3.2 Throttle Capability ............................................................................................. 12

3.3.3 Moment Balance ................................................................................................. 13

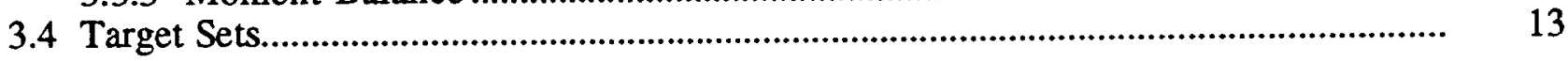

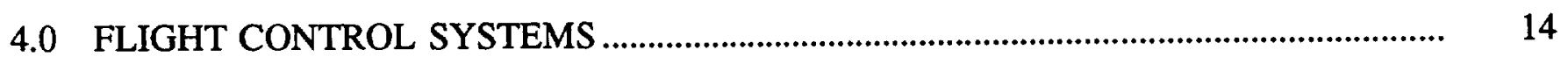

4.1 Reaction Control System Algorithm .............................................................................. 14

4.2 Thrust Vector Controller .............................................................................................. 15

4.3 Aerosurface Controller .................................................................................................. 16

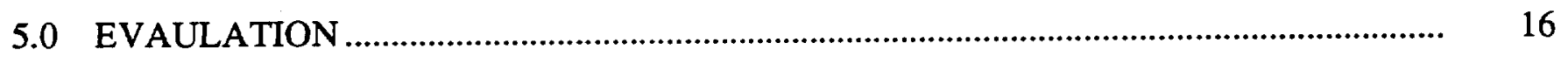

5.1 Guidance Dispersion Analysis .................................................................................... 16

5.1.1 Description ................................................................................................ 16

5.1.2 Results.................................................................................................. 17

5.1.3 Discussion....................................................................................................... 17

5.2 Inversion and Landing Analysis .............................................................................. 19

5.2.1 Aerodynamic Inversion ............................................................................. 20

5.2.1.1 Flight Profile.................................................................................. 20

5.2.1.2 Results ........................................................................................ 20 



\section{TABLE OF CONTENTS (Continued)}

Page

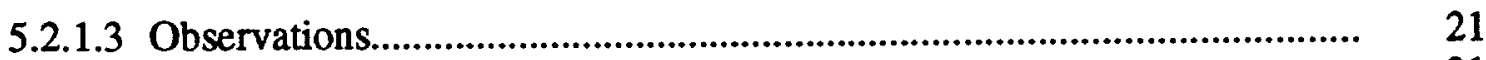

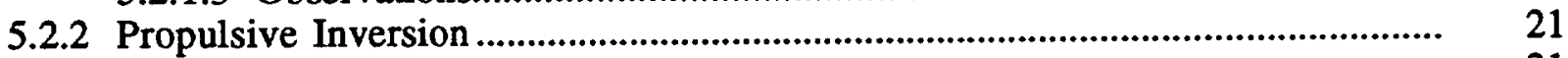

5.2.2.1 Flight Profile............................................................................................ 21

5.2.2.2 Results .......................................................................................... 22

5.2.2.3 Observations........................................................................................ 22

5.2.3 Powered Pullup Inversion ........................................................................................ 22

5.2.3.1 Flight Profile........................................................................................ 22

5.2.3.2 Results ...................................................................................... 23

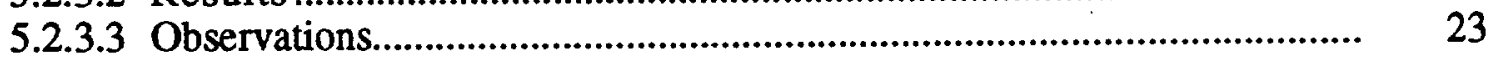

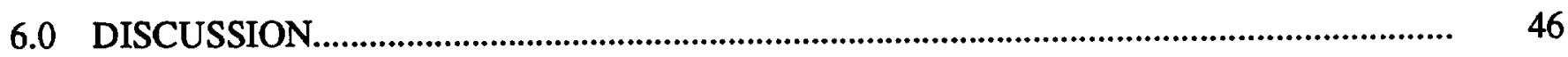

7.0 FUTURE WORK REQUIRED TO SHOW FEASIBILITY ................................................... 48

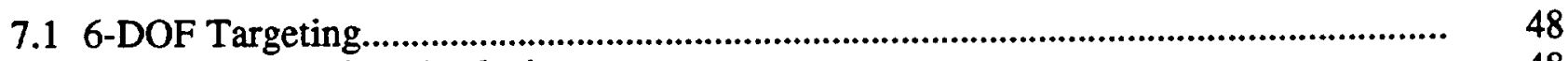

7.2 6-DOF Dispersion Analysis ...................................................................................... 48

7.3 Propulsion System ............................................................................................................. 49

7.3.1 Engine-Out Analysis............................................................................................. 49

7.4 Propellant Management .................................................................................................. 50

7.5 Sensor Dynamics..................................................................................................... 50

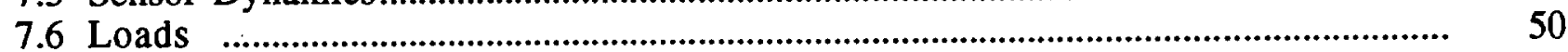

7.7 Aerodynamics ......................................................................................................... 51

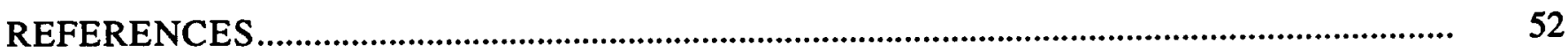





\section{LIST OF ILLUSTRATIONS}

Figure

Title

Page

1. Aerodynamic inversion maneuver..................................................................................... 2

2. Propulsive inversion maneuver................................................................................... 3

3. Powered pullup maneuver ................................................................................................ 3

4. Vertical lander general configuration and layout.............................................................. 5

5. RCS controller phase plane .......................................................................................... 14

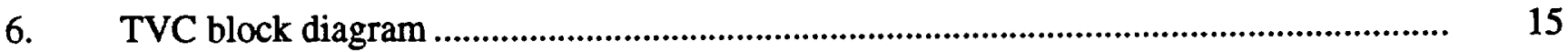

7. VL aerodynamic inversion maneuver, altitude versus time ............................................ 24

8. VL aerodynamic inversion maneuver, altitude rate versus time ..................................... 24

9. VL aerodynamic inversion maneuver, angle of attack versus time ................................ 25

10. VL aerodynamic inversion maneuver, flight path angle versus time............................... 25

11. VL aerodynamic inversion maneuver, Mach number versus time ................................... 26

12. VL aerodynamic inversion maneuver, relative velocity versus time.............................. 26

13. VL aerodynamic inversion maneuver, downrange position versus time......................... 27

14. VL aerodynamic inversion maneuver, downrange velocity versus time ........................ 27

15. VL aerodynamic inversion maneuver, engine throttle versus time ................................. 28

16. VL aerodynamic inversion maneuver, pitch angle and command versus time............... 28

17. VL aerodynamic inversion maneuver, gimbal angle versus time ..................................... 29

18. VL aerodynamic inversion maneuver, main propulsion system propellant usage versus time.

19. VL aerodynamic inversion maneuver, vehicle accelerations versus time...................... 30

20. VL aerodynamic inversion maneuver, aerodynamic normal force versus time .............. 30

21. VL aerodynamic inversion maneuver, RCS torque versus time ..................................... 31

22. VL aerodynamic inversion maneuver, RCS propellant usage versus time .................... 31

vii

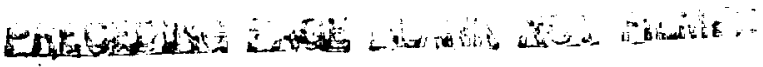




\section{LIST OF ILLUSTRATIONS (Continued)}

Figure

Title

Page

23. VL propulsive inversion maneuver, altitude versus time............................................... 32

24. VL propulsive inversion maneuver, altitude rate versus time........................................ 32

25. VL propulsive inversion maneuver, angle of attack versus time.................................... 33

26. VL propulsive inversion maneuver, flight path angle versus time.................................. 33

27. VL propulsive inversion maneuver, Mach number versus time....................................... 34

28. VL propulsive inversion maneuver, relative velocity versus time.................................. 34

29. VL propulsive inversion maneuver, downrange position versus time............................ 35

30. VL propulsive inversion maneuver, downrange velocity versus time............................ 35

31. VL propulsive inversion maneuver, engine throttle versus time...................................... 36

32. VL propulsive inversion maneuver, pitch angle and command versus time.................. 36

33. VL propulsive inversion maneuver, gimbal angle versus time ....................................... 37

34. VL propulsive inversion maneuver, main propulsion system propellant usage

versus time.

35. VL propulsive inversion maneuver, vehicle accelerations versus time.......................... 38

36. VL propulsive inversion maneuver, aerodynamic normal force versus time................... 38

37. VL pullup maneuver, altitude versus time ......................................................................... 39

38. VL pullup maneuver, altitude rate versus time................................................................. 39

39. VL pullup maneuver, angle of attack versus time............................................................. 40

40. VL pullup maneuver, flight path angle versus time ...................................................... 40

41. VL pullup maneuver, Mach number versus time ........................................................... 41

42. VL pullup maneuver, relative velocity versus time ........................................................... 41

43. VL pullup maneuver, downrange position versus time ................................................... 42

44. VL pullup maneuver, downrange velocity versus time................................................. 42 


\section{LIST OF ILLUSTRATIONS}

Figure

Title

Page

45. VL pullup maneuver, engine throttle versus time................................................................ 43

46. VL pullup maneuver, pitch angle and command versus time ........................................... 43

47. VL pullup maneuver, gimbal angle versus time................................................................. 44

48. VL pullup maneuver, main propulsion system propellant usage versus time................ 44

49. VL pullup maneuver, vehicle accelerations versus time ................................................... 45

50. VL pullup maneuver, aerodynamic normal force versus time............................................ 45

51. Altitude comparison between propulsive inversion and pullup maneuvers ................... $\quad 47$

\section{LIST OF TABLES}

Table

Title

Page

1. Vertical lander mass properties......................................................................................... 5

2. Vertical lander aerodynamic characteristics ................................................................. 6

3. Vertical lander inversion aerodynamic data.................................................................... 6

4. Vertical lander main engine data for landing mode......................................................... 7

5. Vertical lander RCS configuration............................................................................ 8

6. Guidance target sets for three landing options.............................................................. 13

7. Guidance dispersion initial conditions......................................................................... 17

8. Guidance dispersion analysis target sets................................................................... 17

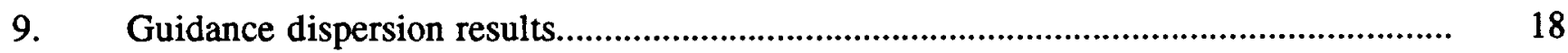



TECHNICAL MEMORANDUM

\section{A GUIDANCE AND CONTROL ASSESSMENT OF THREE VERTICAL LANDING OPTIONS FOR RLV}

\subsection{INTRODUCTION}

From November, 1994, through March, 1995, NASA evaluated a reusable launch vehicle (RLV) vertical lander (VL) candidate concept. The RLV is envisioned to be a completely autonomous vehicle capable of performing a variety of missions including the delivery of payload and/or personnel to the planned International Space Station. The authors, serving as members of MSFC's RLV flight mechanics team, concentrated on guidance and control (G\&C) subsystem design and analysis for the reentry, inversion, and landing phases of the mission. This report documents the primary results of those efforts.

\subsection{Purpose}

Successful design of a VL spacecraft requires early attention to the vertical landing phase itself; that part of the mission which defines the VL's uniqueness. Despite the immaturity of the RLV VL configuration, preliminary simulation and analysis can yield crucial understanding of the unique challenges associated with this concept. The work documented herein has provided valuable insight into many RLV VL design issues, including peculiar problem areas, the required sophistication of the G\&C subsystem, feasibility concerns, and the subsystems likely to receive stringent requirements imposed by the G\&C subsystem.

\subsection{Landing Concepts}

The VL reentry and landing mission phase will consist of several distinct subphases, among them deorbit burns, atmospheric reentry, atmospheric flight (hypersonic to subsonic), maneuver to vertical landing orientation, terminal descent, and landing. Many of these phases reflect familiar, well-understood problems in space vehicles, for which engineers have established reliable approaches to solving. Other phases present unique challenges that aerospace system developers have never addressed in a functional launch vehicle; in particular, the maneuver to vertical orientation and the terminal descent and landing. These phases, tightly constrained by propellant and time considerations, will require closely integrated G\&C algorithm development and implementation.

The integrated G\&C analyses discussed in this report centered upon a single nominal reentry trajectory, providing an initial simulation state (taken from trajectories designed to return the RLV VL from orbit to an altitude of $16,800 \mathrm{~m}$ or $55,000 \mathrm{ft}$ ) and a target landing state (vehicle upright, descending at a sufficiently small rate). The studies considered three variations in the landing mission profile by which the flight control system might perform the inversion of the vehicle (inversion refers to the intentional change from the nose-first reentry attitude to the upright landing attitude). Common to all three concepts is a 10 -s vertical descent to touchdown. This $10-s$ period is desirable to allow time for the vehicle to null any residual attitude rates, obtain and hold a vertical orientation, and null small dispersions such as ground effects and winds at the landing site. 


\subsubsection{Aerodynamic Inversion Maneuver}

The aerodynamic inversion concept (fig. 1) initiates the maneuver at a "high" altitude and subsonic Mach number (as compared to the other two inversion concepts) by utilizing the vehicle's unstable aerodynamic characteristics: upon retraction of aerodynamic control surfaces, aerodynamic moments cause the vehicle to pitch up. Control torque, furnished by a reaction control system (RCS), stabilizes the vehicle in a tail-first attitude and maintains an angle of attack of $180^{\circ}$ until landing guidance is initiated. Upon guidance initiation, the engines are ignited and guidance commands are followed to achieve a vertical touchdown.

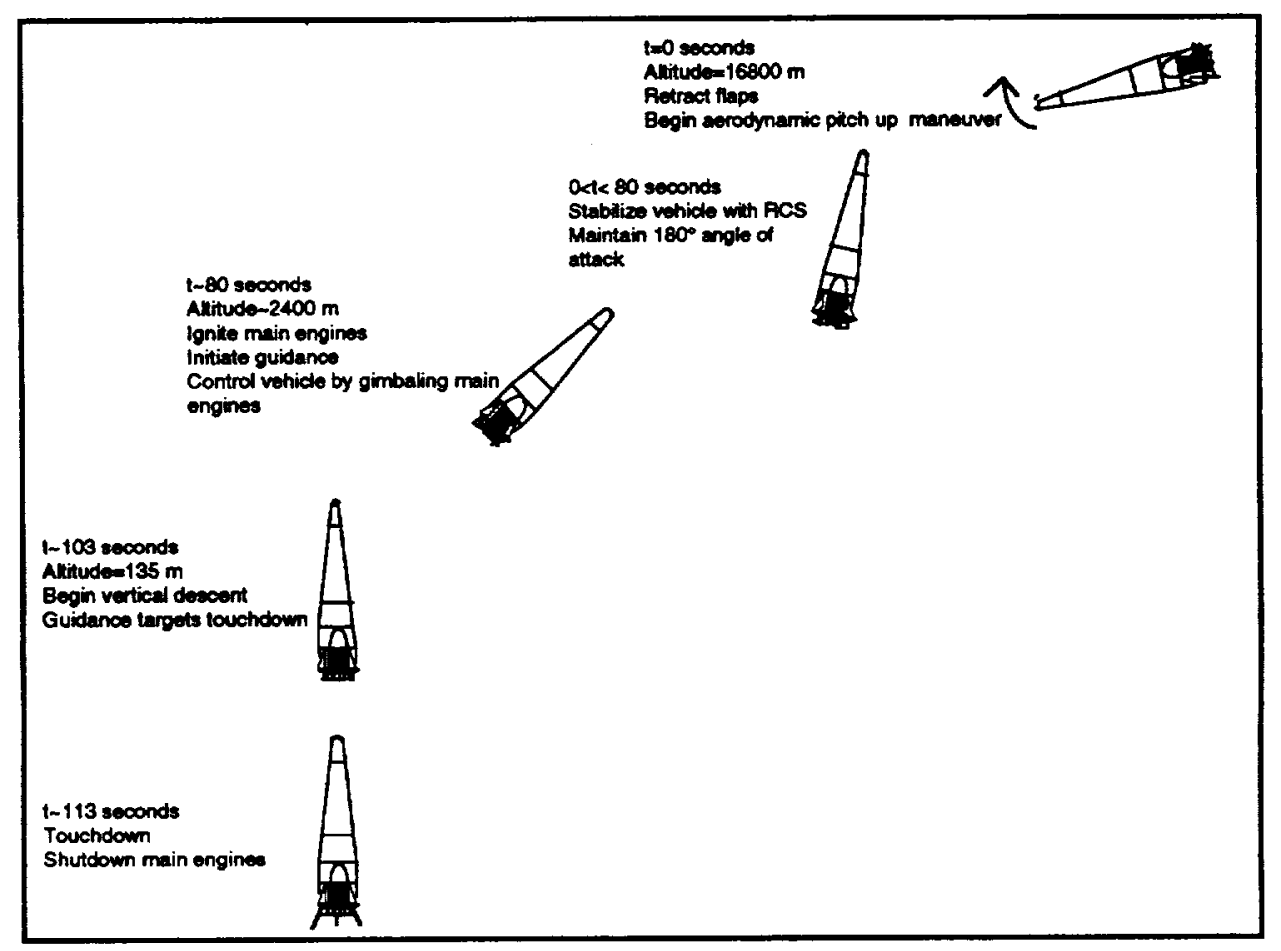

Figure 1. Aerodynamic inversion maneuver.

\subsubsection{Propulsive Inversion Maneuver}

The propulsive inversion concept (fig. 2) requires an inversion initiated at a lower altitude and Mach number. The vehicle allows the aerodynamic moments to cause a positive pitch (as in the previous concept, by retracting aerodynamic control surfaces and temporarily relinquishing attitude control). The vehicle ignites and gimbals the main engines to stabilize the vehicle in a tail-first attitude, while maintaining a negative flight path angle. Guidance attitude and throttle commands are then followed to touchdown.

\subsubsection{Powered Pullup Maneuver}

The powered pullup maneuver concept (fig. 3) begins at an even lower altitude and Mach number. The main engines are then utilized to control the vehicle in a "pullup" maneuver which raises the flight path to a positive angle (above the horizontal). This aspect of the maneuver produces a momentary "hover" point. Guidance commands attitude and throttle to achieve a vertical touchdown. 


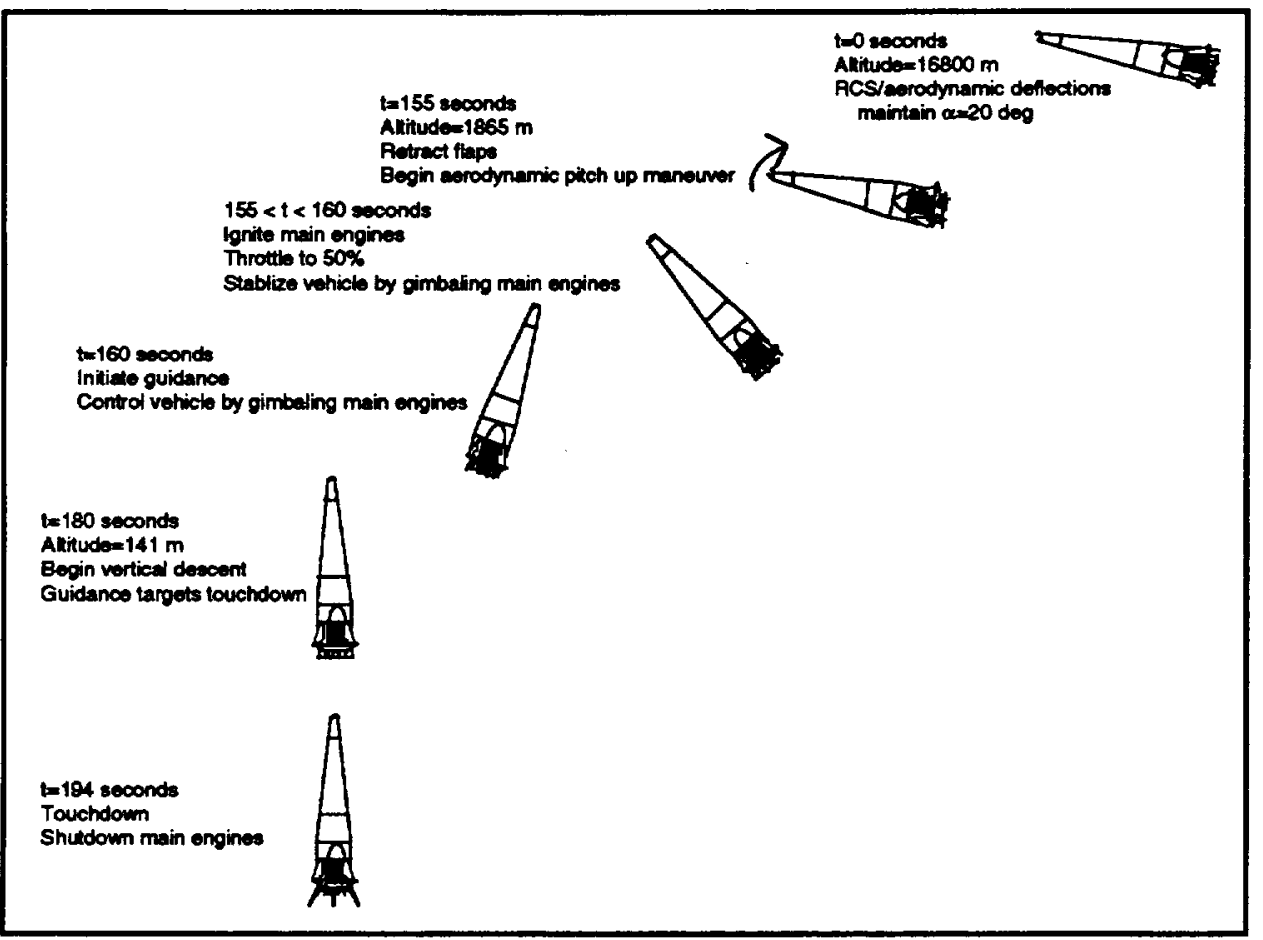

Figure 2. Propulsive inversion maneuver.

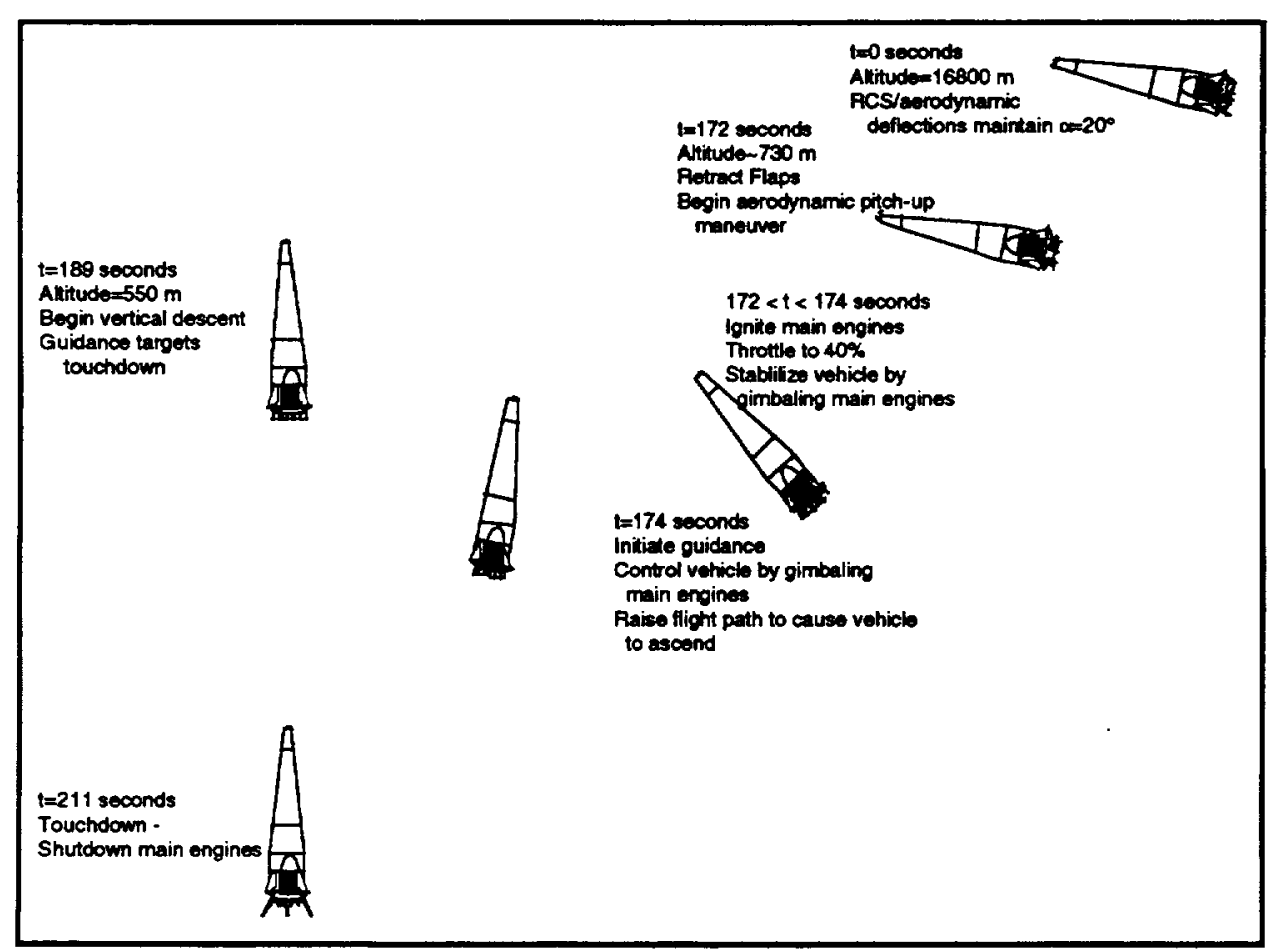

Figure 3. Powered pullup maneuver. 


\subsection{Limitations of the Study}

While this study provides essential insight into the RLV VL design, it does not confirm the viability of any of the three concepts due to significant limitations in the conceptual, structural, and environmental design details available at this time. The integrated G\&C simulations included several parameters for purposes other than strictly realistic evaluation of VL performance:

- Estimated fuel usage provides some measure of relative performance between concepts, but may not reflect realistic usage levels (after maturing guidance and control schemes, accounting for dispersions, etc.).

- Slight differences in touchdown conditions do not represent the relative superiority of one concept over another. Neither should differences in downrange distance suggest any favorability toward any particular concept, since guidance did not attempt to constrain this parameter.

Unavailability of lateral and directional aerodynamic data and lack of detailed mass properties (no cross products of inertia, no migration of center of gravity (cg) with fuel consumption and no offsets of $\mathrm{cg}$ from centerline) constrained the capability to fully assess vehicle dynamics. Although the final simulations modeled six degrees-of-freedom (DOF) (altitude, downrange, crossrange, roll, pitch, and yaw), this lack of data resulted in simulations in effectively reduced degrees-of-freedom (3-DOF becomes 2-DOF for guidance simulations and 6-DOF becomes 3-DOF for the integrated guidance and control simulations).

Lack of information regarding main engine gimbal range and throttle capability led to nonconservative assumptions that reduce the fidelity of the results (unrealistic assumptions, however, could produce equally unreliable results and would introduce additional restrictions and constraints, greatly complicating the analyses).

A wind tunnel test performed at Mach 0.3 for the McDonnell Douglas DC-X vehicle provided the only available aerodynamic data for angles of attack greater than $25^{\circ}$ (by definition, simulation of the inversion requires modeling of this range). Use of these data at all subsonic Mach numbers presumes the insignificance of variations in Mach numbers in the subsonic regime.

\subsection{VEHICLE DESCRIPTION}

Figure 4 depicts the RLV VL vehicle concept, showing the structural coordinate system and main engine locations and number designations.

\subsection{Mass Properties}

Table 1 shows the mass properties used for the inversion and landing assessments. The mass properties presented represent a reentry configuration with a 25,000-lb payload in the payload bay. Some liberty was taken in locating the $\mathrm{x}$-axis $\mathrm{cg}$ as shown in table 1 . Initial analysis of the vehicle's mass properties located the cg further aft in the vehicle (approximately 71.7 percent of the body length measured from the vehicle's nose). This original aft location did not allow the vehicle to achieve suitable trim characteristics when the reentry trim and stabiltiy analyses were performed. Therefore, the $\mathrm{x}$-axis cg was placed, for these analyses, in the most aft location possible (68-percent body length) while still maintaining favorable trim characteristics for the reentry subphases of 

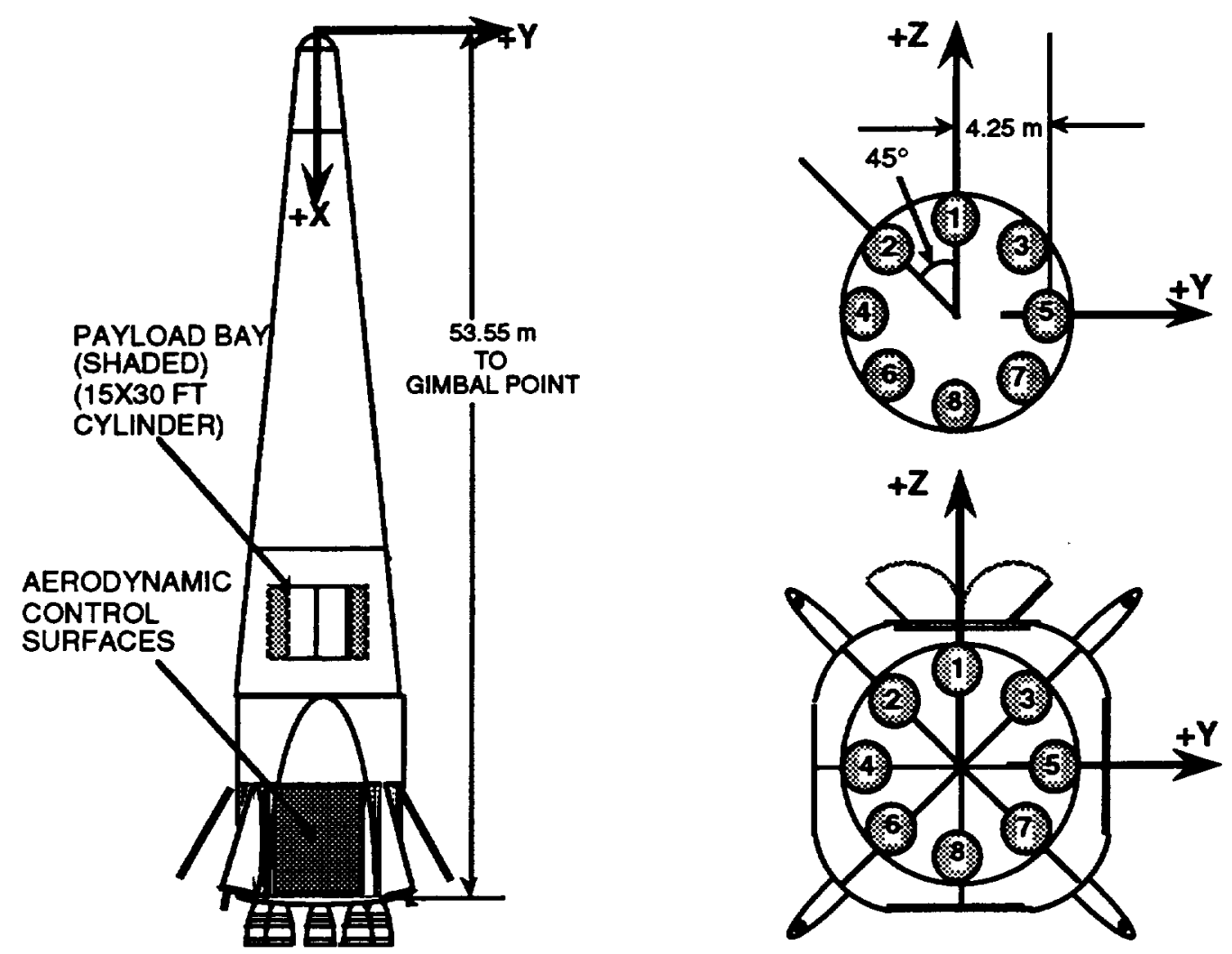

Figure 4. Vertical lander general configuration and layout.

flight. While the integrated G\&C simulations allowed the vehicle's mass to vary as propellant was used, neither the $\mathrm{cg}$ nor the mass moment of inertia was modified to reflect the consumed propellant.

Table 1. Vertical lander mass properties.

\begin{tabular}{|l|l|l|}
\hline Mass/Weight & $129384.5 \mathrm{~kg}$ & $(285,244.0 \mathrm{lb})$ \\
Xcg & $36.4 \mathrm{~m}$ & $(119.4 \mathrm{ft})$ \\
Ycg & $0.0 \mathrm{~m}$ & $(0.0 \mathrm{ft})$ \\
Zcg & $0.0 \mathrm{~m}$ & $(0.0 \mathrm{ft})$ \\
Ixx & $3.136000 .0 \mathrm{~kg}-\mathrm{m}^{2}$ & $\left(2,313,000.0\right.$ slug- $\left.\mathrm{ft}^{2}\right)$ \\
Iyy & $24658261.0 \mathrm{~kg}-\mathrm{m}^{2}$ & $\left(18,187,000.0\right.$ slug- $\left.\mathrm{ft}^{2}\right)$ \\
Izz & $24739610.0 \mathrm{~kg}-\mathrm{m}^{2}$ & $\left(18,247,000.0\right.$ slug- $\left.\mathrm{ft}^{2}\right)$ \\
\hline
\end{tabular}

\subsection{Aerodynamic Data}

Two sets of aerodynamic data are used in the VL studies, one set based on analytic models and one set modified from DC-X wind tunnel data. The analytic data, generated by the Aerodynamic Preliminary Analysis System (APAS) program, provides longitudinal coefficients $\left(C_{A}, C_{N}\right.$, and $\left.C_{m}\right)$ for a small angle-of-attack range $\left(-2.5\right.$ to $\left.25.0^{\circ}\right)$ for all flight phases (hypersonic to subsonic regimes). The APAS data is described fully in reference 1 . The vehicle's aerodynamic characteristics are given in table 2 . 
Table 2. Vertical lander aerodynamic characteristics.

\begin{tabular}{|l|c|c|}
\hline Reference Area & $171.0 \mathrm{~m}^{2}$ & $\left(1840.27 \mathrm{ft}^{2}\right)$ \\
Reference Length & $53.5 \mathrm{~m}$ & $(175.7 \mathrm{ft})$ \\
Moment Reference Point & & \\
Xmrp & $37.49 \mathrm{~m}$ & $(122.99 \mathrm{ft})$ \\
Ymrp & $0.0 \mathrm{~m}$ & $(0.0 \mathrm{ft})$ \\
Zmrp & $0.0 \mathrm{~m}$ & $(0.0 \mathrm{ft})$ \\
\hline
\end{tabular}

The modified wind tunnel data, hereafter referred to as the inversion data set, consist of a full $360^{\circ}$ angle-of-attack range at a Mach number of 0.3 . Time and funding constraints resulted in the previously mentioned unavailability of lateral/directional aerodynamic coefficients. The inversion aerodynamic data is included in table 3.

Table 3. Vertical lander inversion aerodynamic data.

\begin{tabular}{|c|c|c|c|}
\hline $\begin{array}{l}\text { Angle of Attack } \\
\text { (degree) }\end{array}$ & $\begin{array}{c}\text { Normal Force } \\
\text { Coefficient }\end{array}$ & $\begin{array}{l}\text { Axial Force } \\
\text { Coefficient }\end{array}$ & $\begin{array}{l}\text { Pitching Moment } \\
\text { Coefficient }\end{array}$ \\
\hline $\begin{array}{r}-180 \\
-170 \\
-160 \\
-150 \\
-140 \\
-130 \\
-120 \\
-110 \\
-100 \\
-90 \\
-80 \\
-70 \\
-60 \\
-50 \\
-40 \\
-30 \\
-20 \\
-10 \\
0 \\
10 \\
20 \\
30 \\
40 \\
50 \\
60 \\
70 \\
80 \\
90 \\
100 \\
110 \\
120 \\
130 \\
140 \\
150 \\
160 \\
170 \\
180\end{array}$ & $\begin{array}{c}0.0 \\
-0.0835 \\
-0.2069 \\
-0.3702 \\
-0.5435 \\
-0.7168 \\
-0.9101 \\
-1.1335 \\
-1.3568 \\
-1.5937 \\
-1.7747 \\
-1.8474 \\
-1.8037 \\
-1.6502 \\
-1.4058 \\
-1.1068 \\
-0.7862 \\
-0.4148 \\
0.0 \\
0.4148 \\
0.7862 \\
1.1068 \\
1.4058 \\
1.6502 \\
1.8037 \\
1.8474 \\
1.7747 \\
1.5937 \\
1.3568 \\
1.1335 \\
0.9101 \\
0.7168 \\
0.5435 \\
0.3702 \\
0.2069 \\
0.0835 \\
0.0\end{array}$ & $\begin{array}{c}-0.755 \\
-0.681 \\
-0.6089 \\
-0.5446 \\
-0.4793 \\
-0.3587 \\
-0.233 \\
-0.0901 \\
0.0118 \\
0.0763 \\
0.0276 \\
-0.0945 \\
-0.1657 \\
-0.1948 \\
-0.1686 \\
-0.0932 \\
0.0211 \\
0.1319 \\
0.1547 \\
0.1319 \\
0.0211 \\
-0.0932 \\
-0.1686 \\
-0.1948 \\
-0.1657 \\
-0.0945 \\
0.0276 \\
0.0763 \\
0.0118 \\
-0.0901 \\
-0.233 \\
-0.3587 \\
-0.4793 \\
-0.5446 \\
-0.6089 \\
-0.681 \\
-0.755\end{array}$ & $\begin{array}{l}0.0 \\
0.00705 \\
0.01357 \\
0.01826 \\
0.01995 \\
0.01574 \\
0.00643 \\
-0.00945 \\
-0.03146 \\
-0.05539 \\
-0.09799 \\
-0.12908 \\
-0.14499 \\
-0.14404 \\
-0.12666 \\
-0.09356 \\
-0.05004 \\
-0.02246 \\
0.0 \\
0.02246 \\
0.05004 \\
0.09356 \\
0.12666 \\
0.14404 \\
0.14499 \\
0.12908 \\
0.09799 \\
0.05539 \\
0.03146 \\
0.00945 \\
-0.00643 \\
-0.01574 \\
-0.01995 \\
-0.01826 \\
-0.01357 \\
-0.00705 \\
0.0\end{array}$ \\
\hline
\end{tabular}




\subsection{Main Engines}

Eight Russian RD-704 tripropellant engines, rescaled and adapted for the vertical takeoff and landing concept, are used in the main propulsion system. During the two-mode ascent flight phase, the eight engines burn all three propellants $\left(\mathrm{LH}_{2}, \mathrm{RP}-1\right.$, and $\left.\mathrm{LO}_{2}\right)$ in the first mode to provide high propellant bulk density and transition to two propellants $\left(\mathrm{LH}_{2}\right.$ and $\left.\mathrm{LO}_{2}\right)$ in the second mode to provide high specific impulse. For descent and landing, four engines burning two propellants $\left(\mathrm{LH}_{2}\right.$ and $\mathrm{LO}_{2}$ ) provide the required deceleration and are gimbaled to provide three axis attitude control. Figure 4 shows the main engine configuration. Engines 1, 4, 5, and 8 are employed for the landing phase. Table 4 shows the relevant data used to model each engine.

Table 4. Vertical lander main engine data for landing mode.

\begin{tabular}{|l|c|c|}
\hline Vacuum Thrust & $1,165.64 \mathrm{kN}$ & $(262,046 \mathrm{lb})$ \\
Vacuum Specific Impulse & & $(425.4 \mathrm{~s})$ \\
Flow Rate & $279.41 \mathrm{~kg} / \mathrm{s}$ & $(616.0 \mathrm{lb} / \mathrm{s})$ \\
$\quad$ Lox $(86.95$ percent $)$ & $242.95 \mathrm{~kg} / \mathrm{s}$ & $(535.6 \mathrm{lb} / \mathrm{s})$ \\
$\mathrm{LH}_{2}(13.05$ percent$)$ & $36.47 \mathrm{~kg} / \mathrm{s}$ & $(80.4 \mathrm{lb} / \mathrm{s})$ \\
Exit Area & $1.52 \mathrm{~m}^{2}$ & $\left(16.38 \mathrm{ft}^{2}\right)$ \\
\hline
\end{tabular}

\subsection{Thrust Vector Control Actuators}

While specific thrust vector control (TVC) actuators have not been designed or chosen for the VL vehicle, representative dynamics were included in the G\&C simulations to assess the effects of the time and phase lags associated with actual actuators. For the landing assessments, the TVC actuators were modeled with second-order differential equations representing a natural frequency of $4.2 \mathrm{~Hz}$ and a damping ratio of 0.707 .

\subsection{Reaction Control System Thrusters}

An RCS thruster configuration was developed to deliver the required control torque to the vehicle during the aerodynamic inversion maneuver. Two points on the body were chosen as representative placement opportunities, and all thrusters were located at these two points. While this arrangement is physically not realizable, it was assumed, for the purposes of these analyses, that the actual configuration would yield approximately the same torque magnitudes. Sixteen primary RCS thrusters are required (eight for positive torque and eight for negative torque) to provide sufficient torque to reduce the pitching rates and stabilize the vehicle during the aerodynamic inversion maneuver. Ten of the RCS thrusters are positioned at the forward location and six at the aft location. The forces imparted to the body by the thrusters are directed in either the positive or negative $\mathrm{z}$-direction depending on the polarity of the torque required. Table 5 shows the thrust magnitude of the primary thrusters, the specific impulse, and the placement and force directions on the VL vehicle for the aerodynamic inversion concept. 
Table 5. Vertical lander RCS configuration.

Thrust Magnitude Specific Impulse

4,448.2 $\mathrm{N} \quad(1,000.0 \mathrm{lb})$

$(247.0 \mathrm{~s})$

\begin{tabular}{|c|c|c|c|c|c|r|}
\hline Thruster No. & Xloc & Yloc & Zloc & Xdir & Ydir & Zdir \\
\hline 1 & $3.0 \mathrm{~m}(9.8 \mathrm{ft})$ & $0.0 \mathrm{~m}$ & $0.0 \mathrm{~m}$ & 0.0 & 0.0 & 1.0 \\
2 & $3.0 \mathrm{~m}(9.8 \mathrm{ft})$ & $0.0 \mathrm{~m}$ & $0.0 \mathrm{~m}$ & 0.0 & 0.0 & -1.0 \\
3 & $3.0 \mathrm{~m}(9.8 \mathrm{ft})$ & $0.0 \mathrm{~m}$ & $0.0 \mathrm{~m}$ & 0.0 & 0.0 & 1.0 \\
4 & $3.0 \mathrm{~m}(9.8 \mathrm{ft})$ & $0.0 \mathrm{~m}$ & $0.0 \mathrm{~m}$ & 0.0 & 0.0 & -1.0 \\
5 & $3.0 \mathrm{~m}(9.8 \mathrm{ft})$ & $0.0 \mathrm{~m}$ & $0.0 \mathrm{~m}$ & 0.0 & 0.0 & 1.0 \\
6 & $3.0 \mathrm{~m}(9.8 \mathrm{ft})$ & $0.0 \mathrm{~m}$ & $0.0 \mathrm{~m}$ & 0.0 & 0.0 & -1.0 \\
7 & $3.0 \mathrm{~m}(9.8 \mathrm{ft})$ & $0.0 \mathrm{~m}$ & $0.0 \mathrm{~m}$ & 0.0 & 0.0 & 1.0 \\
8 & $3.0 \mathrm{~m}(9.8 \mathrm{ft})$ & $0.0 \mathrm{~m}$ & $0.0 \mathrm{~m}$ & 0.0 & 0.0 & -1.0 \\
9 & $3.0 \mathrm{~m}(9.8 \mathrm{ft})$ & $0.0 \mathrm{~m}$ & $0.0 \mathrm{~m}$ & 0.0 & 0.0 & 1.0 \\
10 & $3.0 \mathrm{~m}(9.8 \mathrm{ft})$ & $0.0 \mathrm{~m}$ & $0.0 \mathrm{~m}$ & 0.0 & 0.0 & -1.0 \\
11 & $50.7 \mathrm{~m}(116.3 \mathrm{ft})$ & $0.0 \mathrm{~m}$ & $0.0 \mathrm{~m}$ & 0.0 & 0.0 & 1.0 \\
12 & $50.7 \mathrm{~m}(116.3 \mathrm{ft})$ & $0.0 \mathrm{~m}$ & $0.0 \mathrm{~m}$ & 0.0 & 0.0 & -1.0 \\
13 & $50.7 \mathrm{~m}(116.3 \mathrm{ft})$ & $0.0 \mathrm{~m}$ & $0.0 \mathrm{~m}$ & 0.0 & 0.0 & 1.0 \\
14 & $50.7 \mathrm{~m}(116.3 \mathrm{ft})$ & $0.0 \mathrm{~m}$ & $0.0 \mathrm{~m}$ & 0.0 & 0.0 & -1.0 \\
15 & $50.7 \mathrm{~m}(116.3 \mathrm{ft})$ & $0.0 \mathrm{~m}$ & $0.0 \mathrm{~m}$ & 0.0 & 0.0 & 1.0 \\
16 & $50.7 \mathrm{~m}(116.3 \mathrm{ft})$ & $0.0 \mathrm{~m}$ & $0.0 \mathrm{~m}$ & 0.0 & 0.0 & -1.0 \\
\hline
\end{tabular}

\subsection{GUIDANCE}

\subsection{Introduction}

The RLV VL is envisioned to be completely autonomous and will likely have many guidance schemes onboard to guide the vehicle through the various phases of flight: ascent, onorbit, rendezvous, reentry, and landing. The ascent and reentry guidance schemes have the advantage of longer durations, and any dispersions encountered can be nulled by the flight computer in future calls to guidance. The landing phase is particularly critical in that the vehicle must, in a very short time, perform an inversion maneuver, ignite the engines, and touch down with little vertical or horizontal velocity and a vertical orientation. A guidance scheme must provide an optimum or near-optimum thrust profile to safely land the vehicle with minimal computation.

This section presents an algorithm based on E-Guidance ${ }^{2}$ which guided the Apollo lunar lander during the lunar descent phase of flight. Given the vehicle current state, and the time duration desired to complete the maneuver, E-Guidance calculates the required thrust acceleration and engine throttle profiles necessary to reach the desired state.

This scheme does not include a payoff function to minimize and, therefore, cannot be considered to provide the optimum thrust profile. However, research shows guided burns computed by E-Guidance were very near-optimum in fuel expenditure ${ }^{2}$. During the Apollo era, this slight nonoptimality was significantly countered by the lack of onboard computation and storage that would have been required to solve the two-point boundary value problem inherent in the calculus-of-variations (COV) (optimum) approach. This again may be attractive to the VL landing problem in terms of computation speed. A typical landing scenario for a vehicle of this mass is on the order of seconds, which lends itself well to an explicit scheme such as E-Guidance. Precomputation of the burn profile with a COV method may not be feasible due to potentially large dispersions in the VL reentry phase of flight. 
The following section provides the development of a modified E-Guidance as used for the VL landing simulations. The thrust acceleration vector in an inertial coordinate system is determined such that the desired final inertial position, velocity, and vehicle attitude are met.

\subsection{Development}

\subsubsection{Thrust Acceleration Profile}

Given the initial vehicle state:

$$
\begin{aligned}
& \bar{r}\left(t=t_{o}\right)=\left[\begin{array}{lll}
x_{o} & y_{o} & z_{o}
\end{array}\right], \\
& \bar{v}\left(t=t_{o}\right)=\left[\begin{array}{lll}
\dot{x}_{o} & \dot{y}_{o} & \dot{z}_{o}
\end{array}\right],
\end{aligned}
$$

determine the required thrust acceleration vector profile $\bar{a}_{r}(t)$ for the duration $t_{0} \leq t \leq T$, such that at the specified time $T$ :

$$
\begin{gathered}
\bar{r}(t=T)=\left[\begin{array}{lll}
x_{T} & y_{T} & z_{T}
\end{array}\right], \\
\bar{v}(t=T)=\left[\begin{array}{lll}
\dot{x}_{T} & \dot{y}_{T} & \dot{z}_{T}
\end{array}\right], \\
\bar{a}_{T}(t=T)=\bar{a}_{T_{D}},
\end{gathered}
$$

where $\bar{a}_{T_{D}}$ is the desired thrust acceleration vector at the final time $T$. The constraint on the final thrust acceleration imposes the desired final attitude constraint. The remainder of this section discusses the development in just one coordinate. At any given time, the vehicle acceleration is:

$$
\ddot{z}(t)=a_{T_{z}}(t)+g_{z},
$$

where $a_{T_{z}}$ and $g_{z}$ are the thrust acceleration and gravitational components in the $z$ direction, respectively. Accelerations due to aerodynamic forces will be discussed in section 3.3.1.

In general:

$$
\int_{t_{0}}^{t} \ddot{z}(s) d s=\dot{z}(t)-\dot{z}\left(t_{0}\right),
$$

so,

Integrate equation (4):

$$
\int_{i_{0}}^{T} \ddot{z}(s) d s=\dot{z}_{T}-\dot{z}_{0} .
$$

$$
\begin{gathered}
\int_{t_{0}}^{T}\left[\int_{t_{0}}^{t} \ddot{z}(s) d s\right] d t=\int_{t_{0}}^{T} \dot{z}(t) d t-\int_{t_{0}}^{T} \dot{z}_{o} d t \\
\quad=\int_{t_{0}}^{T} d z-\int_{t_{0}}^{T} \dot{z}_{o} d t \\
=z_{T}-z_{0}-\dot{z}_{o}^{*}\left(T-t_{o}\right) .
\end{gathered}
$$


Also,

$$
\ddot{z}(T)=a_{T_{z}}(T)+g_{z}(T) .
$$

There are an infinite number of solutions of $z(t)$ to equations (4) to (6). The method of solution is to limit the number of degrees of freedom of $\ddot{z}(t)$ to the number of constraints, namely three. Choose a function:

$$
\ddot{z}(t)=c_{1}{ }^{*} p_{1}(t)+c_{2}{ }^{*} p_{2}(t)+c_{3}{ }^{*} p_{3}(t),
$$

where $p_{i}(t)(i=1-3)$ are preselected functions and $c_{i}(i=1-3)$ are coefficients to be determined. The $p_{i}(t)$ functions must be linearly independent and twice differentiable. Selection of these functions will be discussed in section 3.3.2. Substitute equation (7) into equations (4) to (6).

$$
\begin{gathered}
\dot{z}_{T}-\dot{z}_{o}=\int_{t_{o}}^{T}\left[c_{1}^{*} p_{1}(t)+c_{2} * p_{2}(t)+c_{3} * p_{3}(t)\right] d t \\
=f_{11}^{*} c_{1}+f_{12} * c_{2}+f_{13} * c_{3} \\
z_{T}-z_{o}-\dot{z}_{o} * T_{g o}=\int_{t_{0}}^{T}\left[\int_{t_{o}}^{t}\left[c_{1}^{*} p_{1}(s)+c_{2} * p_{2}(s)+c_{3} * p_{3}(s)\right] d s\right] d t \\
=f_{21}{ }^{*} c_{1}+f_{22} * c_{2}+f_{23} * c_{3} \\
\ddot{z}(T)=c_{1}^{*} p_{1}(T)+c_{2}{ }^{*} p_{2}(T)+c_{3} * p_{3}(T) \\
=f_{31} * c_{1}+f_{32} * c_{2}+f_{33} * c_{3}
\end{gathered}
$$

where,

$$
T_{8 o}=T-t_{o}
$$

The terms $f_{i j}(i, j=1-3)$ can be determined by proper selection of $p_{i}(t)(i=1-3)$.

Rewrite equations (8) to (10) in matrix form:

$$
\left[\begin{array}{lll}
f_{11} & f_{12} & f_{13} \\
f_{21} & f_{22} & f_{23} \\
f_{31} & f_{32} & f_{33}
\end{array}\right]\left[\begin{array}{c}
c_{1} \\
c_{2} \\
c_{3}
\end{array}\right]=\left[\begin{array}{c}
\dot{z}_{T}-\dot{z}_{o} \\
z_{T}-z_{o}-\dot{z}_{o} * T_{g o} \\
\ddot{z}(T)
\end{array}\right] .
$$

Determine the coefficients by matrix inversion (thus the requirement of linear independency of $p_{i}(t)$ ).

$$
\left[\begin{array}{l}
c_{1} \\
c_{2} \\
c_{3}
\end{array}\right]=\left[\begin{array}{lll}
e_{11} & e_{12} & e_{13} \\
e_{21} & e_{22} & e_{23} \\
e_{31} & e_{32} & e_{33}
\end{array}\right]\left[\begin{array}{c}
\dot{z}_{T}-\dot{z}_{o} \\
z_{T}-z_{o}-\dot{z}_{o} * T_{g o} \\
\ddot{z}(T)
\end{array}\right] \text {. }
$$


With the coefficients uniquely determined, from equation (7) one can calculate the $z$-component vehicle acceleration profile:

$$
\ddot{z}(t)=c_{1} * p_{1}(t)+c_{2}^{*} p_{2}(t)+c_{3}^{*} p_{3}(t) .
$$

From equation (3), we determine the required $z$-component thrust acceleration profile:

$$
a_{T_{z}}(t)=\ddot{z}(t)-g_{z} \text {. }
$$

The method is similar for the $x$ - and $y$-components:

$$
\begin{aligned}
& a_{T_{x}}(t)=\ddot{x}(t)-g_{x}, \\
& a_{T_{y}}(t)=\ddot{y}(t)-g_{y} .
\end{aligned}
$$

The total thrust acceleration at a given time is provided by:

$$
\left|\bar{a}_{T}\right|=\sqrt{a_{T_{x}}^{2}+a_{T,}{ }^{2}+a_{T_{z}}{ }^{2}},
$$

and the thrust direction is provided by the direction cosines:

$$
\begin{aligned}
& \cos (\delta)=\frac{a_{T_{s}}}{\left|\overline{a_{T}}\right|}, \\
& \cos (\zeta)=\frac{a_{T_{\gamma}}}{\left|\overline{a_{T}}\right|}, \\
& \cos (\gamma)=\frac{a_{T_{2}}}{\left|\overline{a_{T}}\right|} .
\end{aligned}
$$

The instantaneous thrust acceleration vector in the inertial system is:

$$
\bar{a}_{r}=\left|\bar{a}_{T}\right| *[\cos (\delta) \hat{i}+\cos (\varsigma) \hat{j}+\cos (\gamma) \hat{k}] \text {. }
$$

\subsubsection{Selection of $p_{i}(t)$ Functions}

As discussed previously, the $p_{i}(t)$ functions limit the degrees of freedom to be considered when solving equations (4) to (6). These functions must be linearly independent and twice differentiable. Reference 2 recommends a polynomial function of $(T-t)$. For instance, consider.

$$
\begin{gathered}
p_{1}(t)=1, \\
p_{2}(t)=(T-t), \\
p_{3}(t)=(T-t)^{2},
\end{gathered}
$$


then, substituting into equation (7) yields:

$$
\ddot{z}(t)=c_{1}+c_{2} *(T-t)+c_{2} *(T-t)^{2} .
$$

Substituting equation (17) into equations (8) to (10) and integrating yields the $f_{i j}$ matrix, which is inverted to determine the E-matrix (thus, E-guidance):

$$
\left[\begin{array}{l}
c_{1} \\
c_{2} \\
c_{3}
\end{array}\right]=\left[\begin{array}{ccc}
0 & 0 & 1 \\
\frac{18}{T_{g o}{ }^{2}} & \frac{-24}{T_{80}^{3}} & \frac{-6}{T_{80}} \\
\frac{-24}{T_{80}{ }^{3}} & \frac{36}{T_{80}{ }^{4}} & \frac{6}{T_{80}{ }^{2}}
\end{array}\right]\left[\begin{array}{c}
\dot{z}_{T}-\dot{z}_{o} \\
z_{T}-z_{o}-\dot{z}_{o} * T_{80} \\
\ddot{z}(T)
\end{array}\right] .
$$

Therefore, at each call to guidance, a complete acceleration profile is provided by substituting equation (18) into equation (12):

$$
a_{T_{2}}(t)=c_{1}+c_{2}^{*}(T-t)+c_{3}^{*}(T-t)^{2}-g_{z} .
$$

Note the coefficients in equation (18) will grow very large as $T_{g o}$ approaches zero. This instability can be avoided by not recomputing the steering profile as $t$ approaches $T$. For the 3-DOF analysis presented in this paper, the guidance calculations were not performed for the last $0.5 \mathrm{~s}$ of flight and the last computed solution was accepted.

\subsection{Guidance Discussion}

\subsubsection{Aerodynamic Accelerations}

The above guidance scheme was developed assuming no atmosphere and thus no acceleration due to the aerodynamic forces. Addition of these accelerations is similar to that of the nonlinear gravity acceleration, simply add its contribution to equation (3). However, a candidate aerodynamic acceleration profile must be provided in equation (12), which can be difficult to generate. Moderate success and performance gain has been seen by assuming the aerodynamic acceleration profile to be a linear function from the current value (at call to guidance) to zero at $t=T$. This, however, is not always the case, particularly for a vehicle that performs an inversion maneuver and thus flies through a large range of angle of attack. Without accurate prior knowledge of the aerodynamic acceleration profile, the guidance scheme is much better behaved when the aerodynamic accelerations are simply ignored. As a result, the unanticipated accelerations are accepted, and guidance is allowed to continually recompute the profile as $t$ approaches $T$. Focused study on a particular landing profile would yield predictable, repeatable aerodynamic accelerations which would be formatted into guidance for improved performance.

\subsubsection{Throttle Capability}

Equation (15) provides the thrust acceleration magnitude that is required to meet the desired boundary conditions. There is no inherent constraint on the commanded thrust acceleration being less than the thrust acceleration that is possible from the vehicle. The throttle command is calculated by: 


$$
\eta=\frac{\left|\bar{a}_{T}\right|}{\frac{\text { Thrust }_{\max }}{\text { mass }}}
$$

where $\eta$ is physically constrained by $0 \leq \eta \leq 1$. For flight profiles in which guidance commands a throttle factor greater than 1 for short periods, target conditions are usually met by thrusting in the commanded direction, limiting the throttle to 1 externally, and letting guidance recompute the thrust acceleration profile in future guidance loops.

\subsubsection{Moment Balance}

For large rocket-powered vehicles such as the VL, the moments on the vehicle caused by aerodynamic forces are typically nulled by gimballing the engine(s). The derivation above provides the inertial thrust acceleration vector, not the desired vehicle attitude. The guidance scheme was expanded to include a moment-balance routine which calculates the vehicle attitude and gimbal angles necessary to provide the required thrust acceleration vector while nulling the adverse aerodynamic moments. A bisection method was employed to find the root (angle of attack) which sets the moment equation to zero. Proper flight planning will eliminate the risk of violating maximum engine gimbal angle limits.

\subsection{Target Sets}

A 3-DOF computer simulation was developed to test the guidance scheme and perform dispersion analysis. Results of the dispersion analysis are provided in section 5.1. Common to all three landing scenerio's is a vertical descent of approximately $10 \mathrm{~s}$. Because of this, the guidance scheme requires two target sets for each mission: The first target set includes the desired altitude, velocity, and attitude at the beginning of the vertical descent. The second target set is the actual touchdown conditions at zero altitude. The guidance scheme has the capability to target the downrange position, but that constraint was not exercised for this study. Further 6-DOF dispersion analysis should include the downrange position constraint. Table 6 presents the developed guidance target sets for each of the three landing options.

Table 6. Guidance target sets for three landing options.

\begin{tabular}{|l|c|c|c|c|c|c|c|}
\cline { 2 - 7 } \multicolumn{1}{c|}{} & $x_{T}$ & $\dot{x}_{T}$ & $\ddot{x}_{T}$ & $z_{T}$ & $\dot{z}_{T}$ & $\ddot{z}_{T}$ & $T_{g \circ}$ \\
\cline { 2 - 8 } \multicolumn{1}{c|}{} & $(\mathrm{m})$ & $(\mathrm{m} / \mathrm{s})$ & $\left(\mathrm{m} / \mathrm{s}^{2}\right)$ & $(\mathrm{m})$ & $(\mathrm{m} / \mathrm{s})$ & $\left(\mathrm{m} / \mathrm{s}^{2}\right)$ & $(\mathrm{s})$ \\
\hline Aerodynamic Inversion & & & & & & & \\
\hline Target Set 1 & 141.0 & -28.22 & 12.76 & - & 0. & 0. & 102.85 \\
\hline Target Set 2 & 21.0 & 0. & 10.81 & - & 0. & 0. & 112.85 \\
\hline Propulsive Inversion & & & & & & & \\
\hline Target Set 1 & 141.0 & -28.22 & 12.76 & - & 0. & 0. & 185.0 \\
\hline Target Set 2 & 18.0 & 0. & 10.81 & - & 0. & 0. & 195.0 \\
\hline Powered Pullup & & & & & & & \\
\hline Target Set 1 & 550.0 & 0. & 9.81 & - & 0. & 0. & 189.0 \\
\hline Target Set 2 & 20.0 & -0.5 & 10.81 & - & 0. & 0. & 214.0 \\
\hline
\end{tabular}


The variables $x_{T}, \dot{x}_{T}$, and $\ddot{x}_{T}$ represent the desired (vehicle c.g.)vertical position, velocity, and acceleration at the target time, while $z_{T}, \dot{z}_{T}$, and $\ddot{z}_{T}$ represent the desired downrange position, velocity, and acceleration. Note again that the downrange position is not constrained.

\subsection{FLIGHT CONTROL SYSTEMS}

The flight control system (FCS) provides the means by which the vehicle can autonomously correct errors in its attitude and attitude rate. The FCS consists of a control law, which determines an appropriate corrective action for a given error, and a control effector, which can produce forces and/or moments on the vehicle according to direction from the control law.

The vehicle relies upon three different sets of control effectors, and requires three different control laws for them. One FCS uses the RCS as its control effector, one uses the TVC actuators and main engines, and another uses aerodynamic surfaces. Each concept requires the use of more than one of these flight control systems at different points in the reentry and landing.

\subsection{Reaction Control System Algorithm}

The VL RCS control mode uses phase plane techniques to calculate control torque commands from attitude and attitude rate errors. Attitude and attitude rate errors are generated by subtracting the sensed values from the commanded values. These errors are compared to RCS switching lines in the phase plane (fig. 5) to determine the polarity and level of the required control torque.

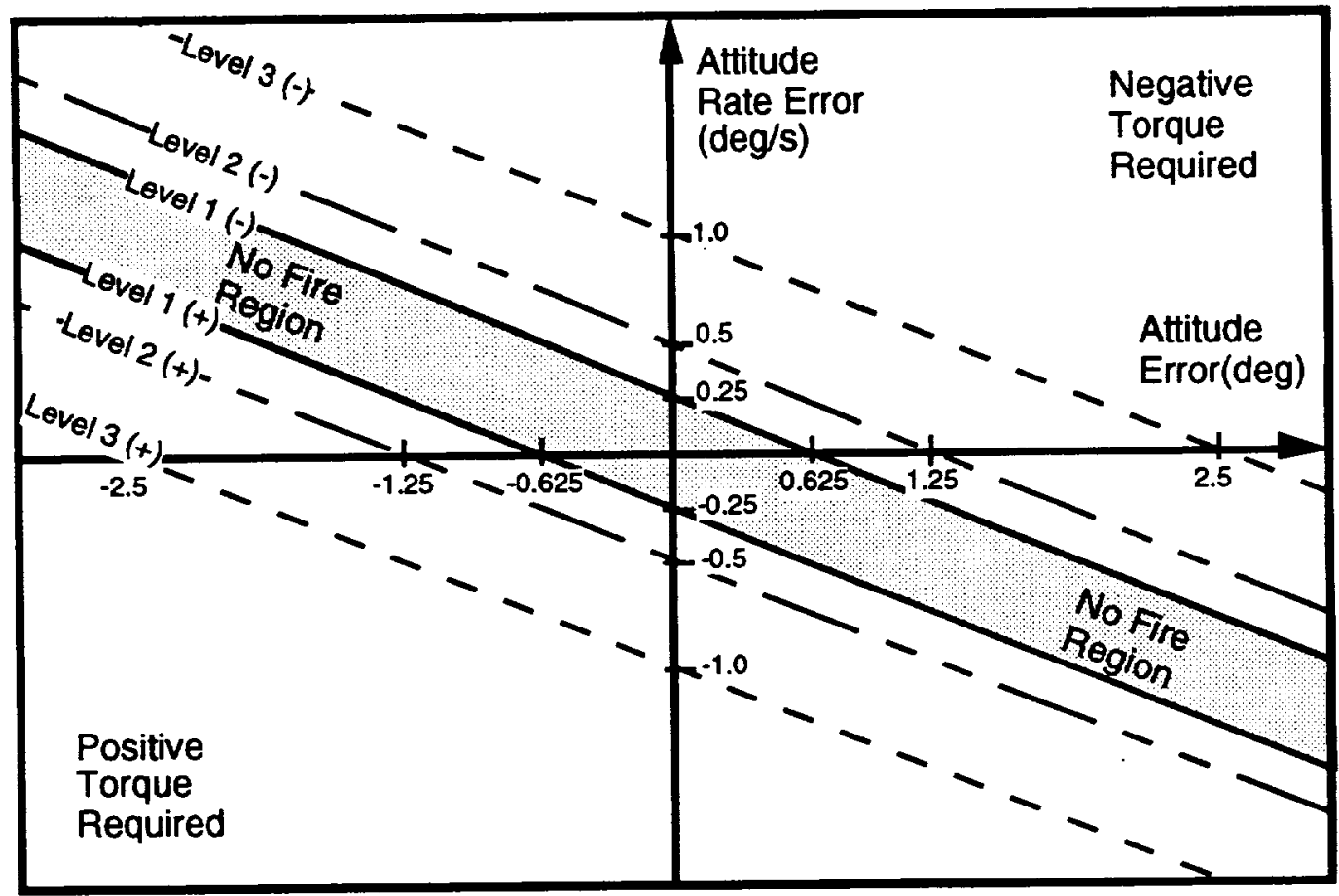

Figure 5. RCS controller phase plane.

Three levels of torque (based on the number of individual thrusters commanded to fire) are used in the RCS control scheme. The lowest torque level is obtained by commanding one forward and one aft thruster to fire. The second torque level commands two forward and two aft thrusters to fire 
and the third level commands all thrusters to fire (five forward and three aft). While increasing the complexity of the control scheme, this multiple level approach reduces the RCS propellant consumption by accounting for the lower torque requirements in attitude holding phases while maintaining high torque capabilities for control during the inversion maneuver.

Figure 5 shows the RCS controller phase plane used in the inversion and landing studies. During each control system cycle, the instantaneous attitude and attitude rate errors, plotted on the phase plane, are compared to the switching lines. The switching lines directly correlate to the torque levels described previously; the first line represents the first torque level and so forth. The required torque polarity is also evident in the phase plane. If the plotted errors are to the right and above the switching lines, the polarity is negative, meaning that negative torque is required to reduce the errors (similarly, positive polarity means that positive torque is required). Note that if the comparison of the attitude and attitude rate errors is not greater than the first switching line, no RCS thrusters are commanded to fire during that control cycle (reducing propellant consumption and allowing a small error deadband). The values for the axes intercepts and switching line slopes were selected based on performance and fuel consumption considerations.

\subsection{Thrust Vector Controller}

The limitations that restricted analysis to only one effective degree of freedom in orientation (pitch angle) simplified the task of designing the TVC controller. The gimbal range of the main engines allow for three-axis control by commanding deflections of each engine individually in two orthogonal directions. The single-axis simplification, however, obviates the need for including this capability in the TVC design. The TVC can simply command all engines to deflect uniformly (in direction and degree) to affect pitch axis control.

A simple "proportional-derivative" structure, as seen in figure 6, proves adequate for the TVC task for all three concepts. In this structure, the negated summation of two error signals yields the pitch angle command. The difference between commanded pitch angle and sensed pitch angle, multiplied by $K_{P}$, forms one error signal. The difference between commanded pitch rate and sensed pitch rate, multiplied by $K_{D}$, forms the other.

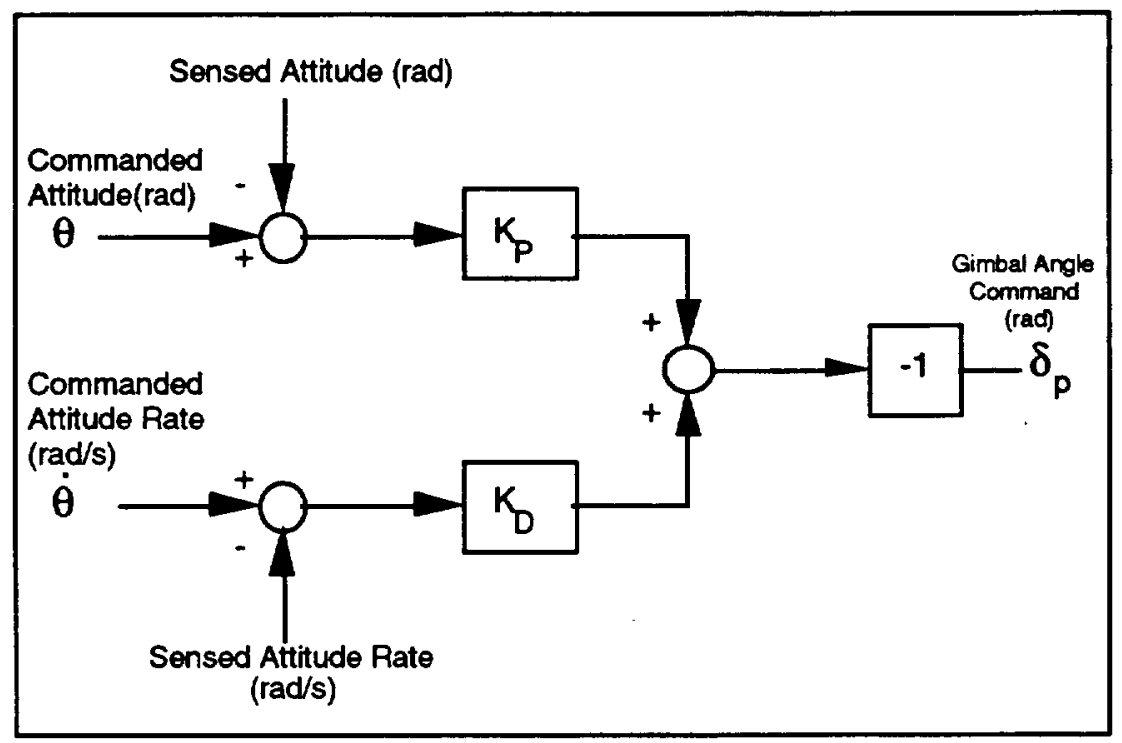

Figure 6. TVC block diagram. 
The selected gains, $K_{P}=10.39$ and $K_{D}=4.68$, yield a controlled system that behaves like a second-order system with a $0.5-\mathrm{Hz}$ bandwidth and 0.707 damping ratio.

Before passing the command, the TVC limits the signal to the appropriate gimbal limits for the concept (propulsive and powered pullup options require greater gimbal range than the aerodynamic inversion) to avoid commanding excessive or impossible deflections.

\subsection{Aerosurface Controller}

The aerosurface controller merely commanded a deflection of the windward body flap in proportion to angle-of-attack error, adjusting the aerodynamic trim angle during the reentry (a full aerosurface control law would require far greater sophistication). For the phases studied herein, this FCS proved very ineffective and did little to ameliorate the error in angle of attack for the propulsive and powered pullup concepts (the aerodynamic inversion did not rely upon the aerosurface FCS). The RCS FCS, with thruster levels boosted by three times, augmented the aerodynamic control surfaces to allow the vehicle to fly at a $20^{\circ}$ angle of attack for extended periods in the propulsive and powered pullup simulations. This level of thrust does not reflect a reasonable RCS configuration; it serves instead to point out that the vehicle requires far more effective aerosurfaces and/or aerodynamic characteristics more favorable to the desired reentry and landing profile.

\subsection{EVALUATION}

\subsection{Guidance Dispersion Analysis}

\subsubsection{Description}

The guidance scheme presented in section 3 was developed and implemented in a 3-DOF trajectory simulation package for testing and dispersion analysis. The simulation code was written in FORTRAN on a DEC ALPHA 7610. A fourth-order Runge-Kutta numerical integrator with a time step of $0.01 \mathrm{~s}$ integrated the translational equations of motion, calling guidance at the rate of $10 \mathrm{~Hz}$. This dispersion analysis was performed assuming two engines with a minimum throttle level of 60 percent. For 3-DOF analysis, this is equivalent to four engines with a minimum throttle level of 30 percent.

The purpose of the dispersion analysis is to test the ability of the guidance scheme to achieve the desired target state in the presence of

- Winds

- Aerodynamic coefficient uncertainties

- Off-nominal thrust

- Off-nominal initial conditions

- Navigation errors.

Navigation errors were subcategorized by altitude to reflect current navigational accuracies. 
The test case selected to perform the dispersion analysis is a propulsive inversion concept with a 10-s vertical descent. Table 7 presents the 3-DOF simulation initial conditions for this analysis.

Table 7. Guidance dispersion initial conditions.

\begin{tabular}{|l|l|}
\hline Altitude & $663.6 \mathrm{~m}$ \\
Altitude rate & $-53.2 \mathrm{~m} / \mathrm{s}$ \\
Downrange & $479.7 \mathrm{~m}$ \\
Downrange rate & $-96.3 \mathrm{~m} / \mathrm{s}$ \\
\hline
\end{tabular}

The simulation begins at the completion of the propulsive inversion maneuver (first call to guidance). The guidance scheme targets two states; the condition prior to vertical descent and touchdown. Table 8 presents the guidance dispersion analysis target sets.

Table 8. Guidance dispersion analysis target sets.

\begin{tabular}{|c|c|c|c|c|c|c|c|}
\cline { 2 - 7 } \multicolumn{1}{c|}{} & $\begin{array}{c}x_{T} \\
(\mathrm{~m})\end{array}$ & $\begin{array}{c}\dot{x}_{T} \\
(\mathrm{~m} / \mathrm{s})\end{array}$ & $\begin{array}{c}\ddot{x}_{T} \\
\left(\mathrm{~m} / \mathrm{s}^{2}\right)\end{array}$ & $\begin{array}{c}z_{T} \\
(\mathrm{~m})\end{array}$ & $\begin{array}{c}\dot{z}_{T} \\
(\mathrm{~m} / \mathrm{s})\end{array}$ & $\begin{array}{c}\ddot{z}_{T} \\
\left(\mathrm{~m} / \mathrm{s}^{2}\right)\end{array}$ & $\begin{array}{c}T_{g \circ} \\
(\mathrm{s})\end{array}$ \\
\hline Propulsive Inversion & & & & & & & \\
\hline Target Set 1 & 141.0 & -28.2 & 12.8 & - & 0. & 0. & 12.8 \\
\hline Target Set 2 & 0. & -0.5 & 10.8 & - & 0. & 0. & 10. \\
\hline
\end{tabular}

As described in section 3 , the variables $x_{T}, \dot{x}_{T}$, and $\ddot{x}_{T}$ represent the desired vertical position, velocity, and acceleration at the target time, while $z_{T}, \dot{z}_{T}$, and $\ddot{z}_{T}$ represent the desired downrange position, velocity and acceleration. Note the 3-DOF simulation only tracks the vehicle's cg and therefore touchdown occurs at $x_{T}=0$ (the 6-DOF simulation tracks both the vehicle's cg and landing pods to determine touchdown). Also, as discussed in section 3 , the guidance scheme has the capability to target the downrange position but that option was not utilized here.

\subsubsection{Results}

Table 9 shows the results of the 3-DOF dispersion analysis. The leftmost vertical column lists the dispersion category and the dispersion/uncertainty value. The results include the commanded maximum and minimum throttle value, final target conditions, propellant consumed, and maximum throttle and pitch rates.

\subsubsection{Discussion}

The results presented in table 9 show this guidance scheme to be very robust in the presence of dispersions and uncertainties. The top row is the nominal case (no dispersions). The first two columns show, at some time in each run, that the guidance scheme commanded a throttle value lower than 60 percent and greater than 100 percent. In this situation, the vehicle rotates to the commanded attitude, and the throttle value is externally bounded by 60 and 100 percent. The temporary thrust loss/gain is accepted, and the guidance scheme is permitted to continually recompute the thrust acceleration profile based on its current state. Typical flight simulation showed the throttle command "riding" the bounds for just a few seconds. 


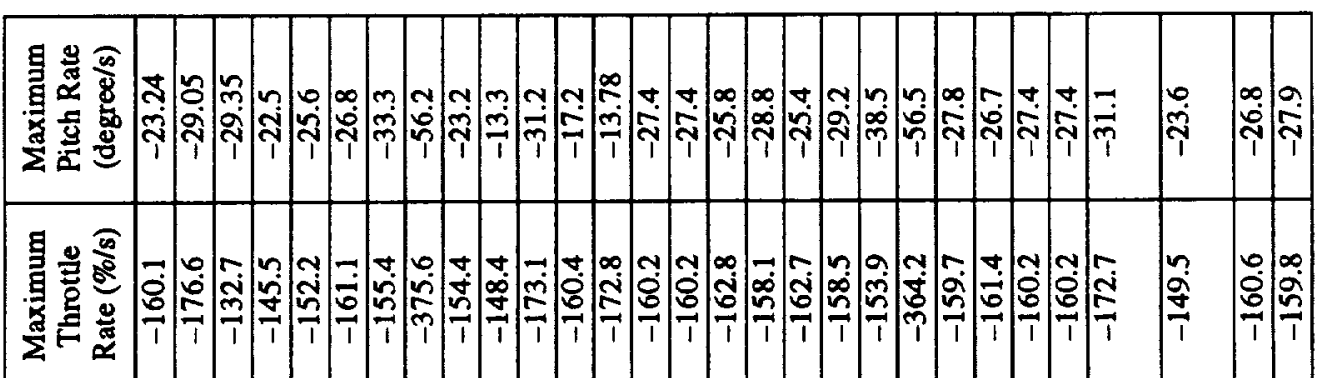

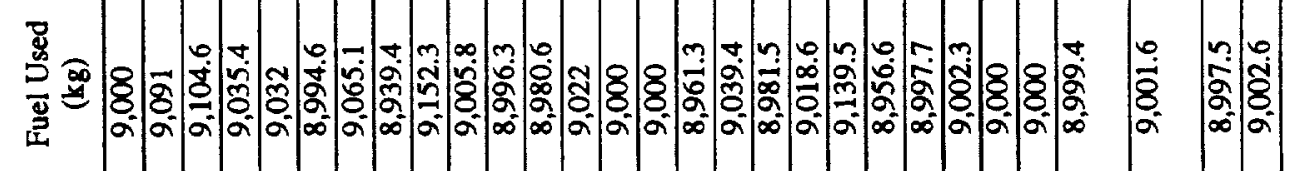

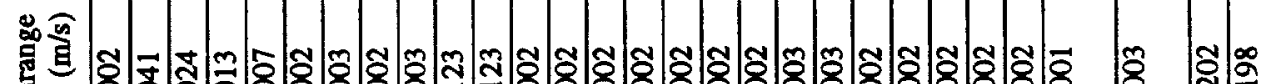

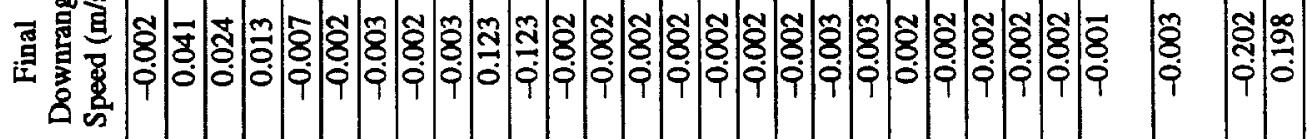

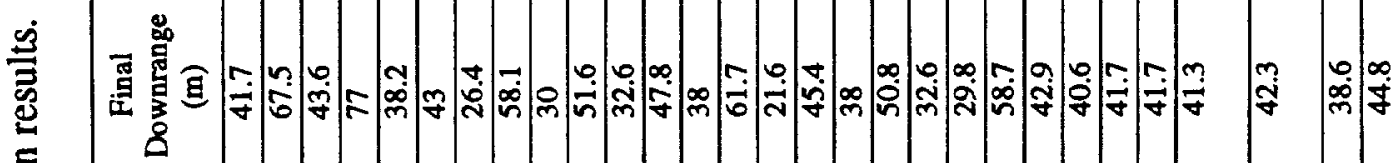

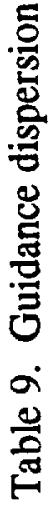

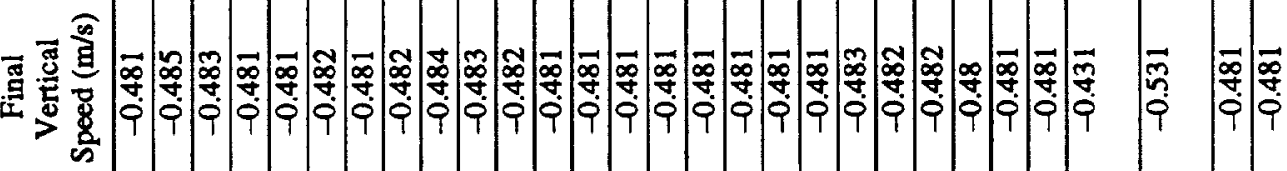

焉意

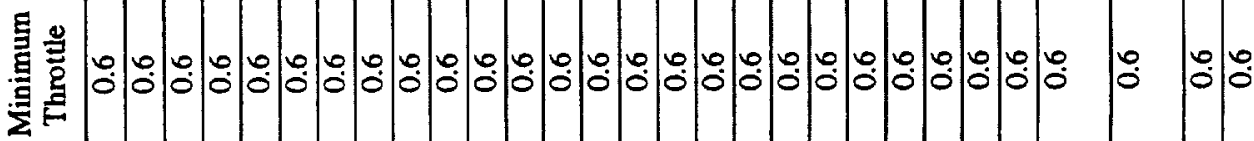

䂓意

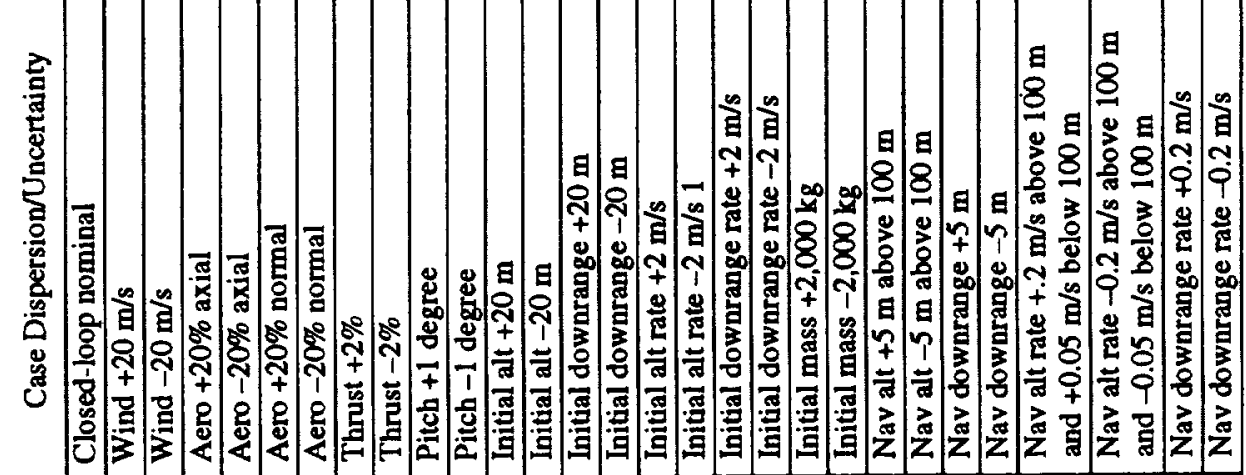


The next column displays the final trajectory altitude; this is provided simply for completeness. The largest dispersion in final vertical speed is only $0.05 \mathrm{~m} / \mathrm{s}$, which occurs when the navigation system, below an altitude of $100 \mathrm{~m}$, sends erroneous altitude rate data to guidance. The next column shows the final downrange position for each of the dispersion runs. Note that even though the downrange position was not constrained, the largest downrange error was only $37 \mathrm{~m}$. This downrange error is a result of a 20-percent error in prediction of the axial aerodynamic coefficients.

The next column shows the final horizontal speed for the various dispersions and uncertainties. It is desired to have zero horizontal speed at touchdown, which is the case with most of the dispersion cases. The largest dispersion in final horizontal speed occurs when the vehicle navigation system senses or calculates a downrange velocity error of $0.2 \mathrm{~m} / \mathrm{s}$. An increase in final downrange velocity is also seen if the vehicle navigation system calculates the pitch angle with an error of $1.0^{\circ}$.

As provided in the first row, the nominal propellant usage is $9,000 \mathrm{~kg}$. The two largest propellant requirements as a result of dispersions and uncertainties occur when there exists a thrust loss of 2 percent and when the initial mass is $2,000 \mathrm{~kg}$ greater than nominal.

The last two columns list the maximum commanded throttle rate and pitch rate. For this propulsive inversion maneuver and target conditions, guidance commanded large throttle and pitch rates at the time just prior to achieving the first target set (prior to the vertical descent). In the 3-DOF dispersion analysis, the guidance scheme did not limit these rates.

\subsection{Inversion and Landing Analyses}

In this section, 6-DOF simulations of each of the three concepts are presented and discussed. These three sets of results are not intended to serve as a "fly-off," or selection of a single concept as being the superior or preferred option. Barring detection of utter infeasibilities, the state of the system design and extent of data would not allow for conclusive selection or rejection of any of the three inversion options. These studies are investigations of each concept individually. Comparisons of the characteristics of each option can be used to guide future trade studies and subsystem development. Primary among the goals of these analyses is determination of the viability of each concept, in order to rule out infeasible options early. The studies also facilitate identification of problem areas and peculiar subsystem requirements unique to a given concept.

The integrated G\&C simulations were each begun from the same initial conditions. Each option, from this reentry point to touchdown, were simulated using Land Upright/Land Unharmed (LULU), a 6-DOF program coded in FORTRAN. LULU integrates the system dynamics using a fourth-order Runge-Kutta algorithm. Winds were not included in the simulated environment because, given the current level of detail in the vehicle data and design, they would have clouded the results more than they clarified them. LULU used a flat Earth model to simplify implementation of guidance by allowing the single angular degree of freedom to be expressed relative to the inertial coordinate system.

For these simulations, the main engines were assumed capable of instantaneous throttle changes, ranging from 10 to 100 percent. It was assumed that engines could be gimballed in a cone of $30^{\circ}$ half-angle. Specifications comparable to these assumptions are probably not realistic for a physical engine. Simulated characteristics and engine capabilities must converge to realistic values as vehicle design, engine design, and G\&C subsystem design mature. 
Guidance and flight control algorithms were developed separately, then integrated for analysis by simulation. Initial 6-DOF simulations were begun at the initial condition and maintained a $20^{\circ}$ angle of attack to an altitude of zero. This trajectory provided vehicle states where each of the maneuvers could be simulated with the 3-DOF guidance test bed. Progressive refinements to the EGuidance implementation and adjustments of parameters yielded a set of target values for each concept with which the 3-DOF was successful in meeting propellant and landing constraints. These target sets were integrated into LULU ${ }^{\mathrm{TM}}$ and further adjusted (along with refinements to the control systems) until the propellant and landing constraints could be met with the additional complexity of the angular degree of freedom associated with the 6-DOF simulation.

In each 6-DOF simulation, guidance commands are updated every $0.5 \mathrm{~s}(2 \mathrm{~Hz})$. This rate is lower than the rate assumed during guidance design, but the lower rate does not appear to be detrimental to performance. Each FCS command is updated every $0.04 \mathrm{~s}(25 \mathrm{~Hz})$. This difference in computational rate, which is typical of other space vehicles, allows the FCS to more smoothly follow guidance commands. The FCS overrides guidance attitude and attitude rate commands below altitudes of $25 \mathrm{~m}$ (30 m in the powered pullup case). This prevents inappropriate attitude adjustments during the vertical descent. A more refined G\&C system would not require this handoff of responsibility between subsystems.

All times listed or shown in the figures in the remainder of this section are referenced to the time at the simulation initial condition $(t=0 \mathrm{~s})$. As previously noted, a flat Earth model was assumed. A planar coordinate system is defined at $t=0$ such that the $\mathrm{x}$-axis is perpendicular to the ground directed upward. The $\mathrm{z}$-axis is parallel to the ground in the direction of the initial vehicle horizontal velocity. Downrange is measured from the reentry/landing interface at altitude $=16,800 \mathrm{~m}$. "Attitude" refers to the pitch angle measured positive from the $\mathrm{x}$-axis toward the $\mathrm{z}$-axis.

\subsubsection{Aerodynamic Inversion}

\subsubsection{Flight Profile}

The simulation begins at $16,800 \mathrm{~m}$ with the vehicle at an angle of attack of $20^{\circ}$. The FCS immediately retracts the aerodynamic control surfaces initiating the inversion maneuver. The RCS thrusters apply torque to oppose the pitch rate as aerodynamic moments drive the vehicle past $180^{\circ}$ angle of attack (at $9.5 \mathrm{~s}$ ) to a maximum overshoot of $-60^{\circ}\left(120^{\circ}\right.$ past the target of $180^{\circ}$ at $\left.15 \mathrm{~s}\right)$. The FCS dampens the residual oscillations with the RCS thrusters until the vehicle attitude settles at $180^{\circ}$ angle of attack (at $39 \mathrm{~s}$ ). The vehicle maintains this attitude for approximately 40 additional seconds. The main engines are ignited and guidance is initiated at $80 \mathrm{~s}$ (approximately $2,400-\mathrm{m}$ altitude), targeting the state prior to vertical descent. At this time, the RCS thrusters are deactivated and the FCS transitions to TVC mode. Guidance commands main engine throttle settings and desired attitude, which is followed by the FCS. At $103 \mathrm{~s}$, the guidance system transitions to target the touchdown state. Touchdown occurs at $112.5 \mathrm{~s}$.

\subsubsection{Results}

Figures 7 through 22 show the integrated G\&C simulation results of the aerodynamic inversion maneuver. Figure 9 shows the large angle-of-attack overshoot and settling time during the inversion maneuver. Initially, the RCS FCS design attempted to achieve the inversion performance claimed by the McDonnell Douglas DC-Y concept (inversion complete and stable in $28 \mathrm{~s}$ ). The control torques required to achieve this performance with the VL configuration were unrealistic (on the order of $1,000,000 \mathrm{ft}-\mathrm{lb}$ ), therefore a more realistic RCS configuration was developed (presented in 
section 2.5) and the FCS design was not driven strictly by performance. Figure 21 shows the multiple RCS torque levels applied to the vehicle as commanded by the RCS controller. Figure 16, displays the commanded and sensed attitude angle from guidance initiation to touchdown. At $103 \mathrm{~s}$, a minimal disturbance in attitude command can be seen as a result of the transition to the second target set within guidance.

\subsubsection{Observations}

This concept requires that large control torques, supplied by either RCS or aerodynamic control surfaces, counter the aerodynamic moments imparted to the vehicle during the inversion maneuver. With the large control torque provided by the RCS configuration developed for this study, the FCS was able to stabilize the vehicle before engine ignition, but was insufficient to prevent the large oscillations in angle of attack that followed the inversion maneuver.

This maneuver used only about $10,000 \mathrm{~kg}$ of the allocated $14,500 \mathrm{~kg}$ of landing propellant. Because this concept orients the vehicle into a tail-first attitude at a fairly high altitude, significant energy is dissipated as a result of the high aerodynamic drag, thus reducing the required thrust acceleration to land the vehicle. Also, the thrust acceleration that is applied is done so appropriately along the (negative) velocity vector for a majority of the flight. These two factors are primarily responsible for the lower propellant consumption associated with this concept.

This option provides a significant period of flight time in a tail-first attitude. This orientation of the vehicle's acceleration vector should tend to locate the propellants in the aft ends of their respective tanks prior to engine ignition.

With an aerodynamic inversion, the vehicle, by maintaining a $180^{\circ}$ angle of attack before initiating guidance, has begun to decrease the horizontal velocity and lower the flight path to vertical. Because of this, the reductions in horizontal velocity required by guidance can be accomplished with relatively small commands to the vehicle attitude. Therefore, the attitude command profile does not contain particularly abrupt changes in direction or magnitude. Such profiles are easily followed by the FCS, so the required gimbal range for this option was a $10^{\circ}$ half-angle cone, rather than the $30^{\circ}$ originally selected for the TVC system in this study.

\subsubsection{Propulsive Inversion}

\subsubsection{Flight Profile}

The simulation begins at $16,800 \mathrm{~m}$ with the vehicle at an angle of attack of $20^{\circ}$. The FCS maintains this attitude using a combination of RCS and aerodynamic control surfaces. At 1,865 m $(t=155 \mathrm{~s})$, the FCS commands retraction of the aerodynamic control surfaces, initiating the inversion maneuver. At this time the main engines are ignited and throttled to 50 percent, and, after deactivating the RCS and switching to TVC mode, the FCS commands a constant pitch rate of 0.3 radians per second (approximately $17 \%$ ). After $2 \mathrm{~s}$ more (at $157 \mathrm{~s}$ ), the FCS commands a zero pitch rate and a pitch attitude of $40^{\circ}$ in order to terminate the inversion maneuver and quickly stabilize the vehicle before activating guidance. At $160 \mathrm{~s}$, guidance is initiated and commands main engine throttle settings and desired attitude, which is followed by the FCS. At $185 \mathrm{~s}$, the guidance system transitions to target the touchdown state. Touchdown occurs at about $194 \mathrm{~s}$. 


\subsubsection{Results}

Figures 23 through 36 show the results of the integrated G\&C simulation of the propulsive inversion. Figure 25 depicts the change in angle of attack during inversion, and figure 26 shows the flight path angle, which remains negative (indicating the vehicle is in constant descent). Figure 32 shows the commanded and sensed attitude angle. Between 157 and $169 \mathrm{~s}$, the attitude does not follow the command closely, showing significant overshoot. The reasons will be discussed in the next section.

\subsubsection{Observations}

Since the main engines are ignited prior to performing the propulsive maneuver, the inversion causes significant disturbance to the flight path angle. This disturbance introduces additional horizontal velocity, for which guidance must account by commanding changes in vehicle attitude. Consequently, the attitude command profile includes an abrupt change at the start of the inversion. In addition, the disturbance causes a decrease in descent rate. Guidance compensates for this by commanding a lower throttle. The lower throttle level greatly reduces the control torque available by gimballing the main engines. Consequently, the FCS greatly overshoots the commanded attitude due to its lessened capacity (at higher throttle levels, the FCS would have been able to stabilize the vehicle more quickly after inversion, with relatively little overshoot). Once the error in descent rate has been corrected, the FCS attains and follows the commanded attitude closely until touchdown.

With this option, the VL must maintain $20^{\circ}$ angle of attack for a significant period of flight. For simulation purposes, this was accomplished, as previously noted, by increasing the RCS thrust level by three times, compensating for an aerodynamic control surface that proved inadequate for such a task. Unless the trajectory and/or the aerodynamic characteristics of the vehicle and its aerosurfaces are changed significantly, tremendous control torque will be necessary to perform this segment of the reentry.

In this simulation, the estimated fuel usage was about $14,000 \mathrm{~kg}$, out of $14,500 \mathrm{~kg}$ usable propellant. A significant portion of this fuel was expended in nulling horizontal velocity imparted by engine thrust during the inversion. This fuel usage does not include the RCS fuel that would be required for augmenting the aerosurface control (as described immediately above).

This option requires that the vehicle initiate the inversion after a significant period of flight in a nose-first attitude. This orientation of the vehicle's acceleration vector should tend to locate the propellants in the forward ends of their respective tanks prior to engine ignition.

\subsubsection{Powered Pullup Inversion}

\subsubsection{Flight Profile}

The simulation begins at $16,800 \mathrm{~m}$ with the vehicle at an angle of attack of $20^{\circ}$. The FCS maintains this attitude using a combination of RCS and aerodynamic control surfaces. Approximately $172 \mathrm{~s}$ later, the FCS commands retraction of the aerodynamic control surfaces, initiating the inversion maneuver. This occurs at an altitude of about $550 \mathrm{~m}$. At this time, the main engines are ignited and throttled to 40 percent, and, after deactivating the RCS and switching to TVC mode, the FCS commands a zero pitch rate and a constant pitch attitude of $-20^{\circ}$. After $2 \mathrm{~s}$ more (at $174 \mathrm{~s}$ ), guidance is initiated and commands main engine throttle settings and desired attitude, which is followed by the FCS. During this phase, guidance directs the FCS (by commanding changes in the pitch attitude) 
to raise the flight path angle to a positive value, which causes the vehicle to slow its descent, then actually ascend and approach a stall condition before continuing with a nearly vertical descent. At $189 \mathrm{~s}$, the guidance system transitions to target the touchdown state. Touchdown occurs at about $211 \mathrm{~s}$.

\subsubsection{Results}

Figures 37 through 50 show the results of the integrated G\&C simulation of the powered pullup maneuver. Figure 39 shows the angle-of-attack profile, which differs markedly in character from those of the other two concepts in that it does not invert to $180^{\circ}$ immediately upon initiation of the maneuver. Rather, it follows an angle-of-attack profile between $90^{\circ}$ and $130^{\circ}$ during the pullup, which can be most easily detected in the flight path angle profile seen on the same figure. The flight path angle (fig. 40) increases to positive values (indicating ascent during the pullup) and drops quickly and settles about $-90^{\circ}$ (reflecting a vertical descent), at which time the angle of attack settles to $180^{\circ}$, confirming tail-first approach.

Figure 46 shows the commanded and sensed attitude profile. The command is followed closely until retargeting of guidance causes an abrupt change in command (from $30^{\circ}$ to nearly $0^{\circ}$ at $189 \mathrm{~s}$ ). This results in about $15^{\circ}$ overshoot, but the FCS regains close tracking in about $5 \mathrm{~s}$, and holds the vehicle very closely to a vertical attitude for about $18 \mathrm{~s}$ more, until touchdown.

\subsubsection{Observations}

In the simulation of this option, the vehicle attitude attains the commanded attitude before a complete inversion has occurred. The FCS follows the guidance commands satisfactorily as guidance raises the flight path angle (imparting some additional horizontal velocity as a consequence). During this pullup, the vehicle moves from the nose-first entry attitude up to $30^{\circ}$ past the vertical landing attitude, while the angle of attack changes from $20^{\circ}$ to $180^{\circ}$ (with some overshoot). When guidance transitions to its second target set, the vehicle is at its maximum guidance-commanded attitude $\left(30^{\circ}\right)$ in order to null horizontal velocity. The abrupt change in attitude command (from $30^{\circ}$ to nearly vertical) results in some attitude overshoot, which is exacerbated because the commanded throttle level is near or at the minimum level of 10 percent, greatly reducing control authority. The FCS recovers as throttle level increases and successfully regains the desired descent and landing attitude.

As with the previous option, the VL must maintain a $20^{\circ}$ angle of attack for a significant period of flight. For simulation purposes, this was accomplished, again, by increasing the RCS thrust level by three times, compensating for an aerodynamic control surface that proved inadequate for such a task. Unless the trajectory and/or the aerodynamic characteristics of the vehicle and its aerosurfaces are changed significantly, tremendous control torque will be necessary to perform this segment of the reentry.

In this simulation, the estimated fuel usage was about $14,450 \mathrm{~kg}$, out of $14,500 \mathrm{~kg}$ usable propellant. Approximately half of this propellant is expended in raising the vehicle flight path angle in the pullup and nulling the resultant horizontal velocity, with the rest spent on controlling descent rate. This fuel usage does not include the RCS fuel that would be required for augmenting the aerosurface control, just as for the propulsive option discussed.

Meeting the budgeted propellant usage in this phase required the maneuver to be initiated at a much lower altitude than was initially investigated. This lower initial altitude results in a lower 
altitude at the start of the final descent. Since, for this option, the final descent is (to the vehicle) much like being dropped from a standstill, a lower altitude is, in most cases, preferable in terms of fuel used to decelerate.

This option requires that the vehicle initiate the inversion after a significant period of flight in a nose-first attitude. This orientation of the vehicle's acceleration vector should tend to locate the propellants in the forward ends of their respective tanks prior to engine ignition.

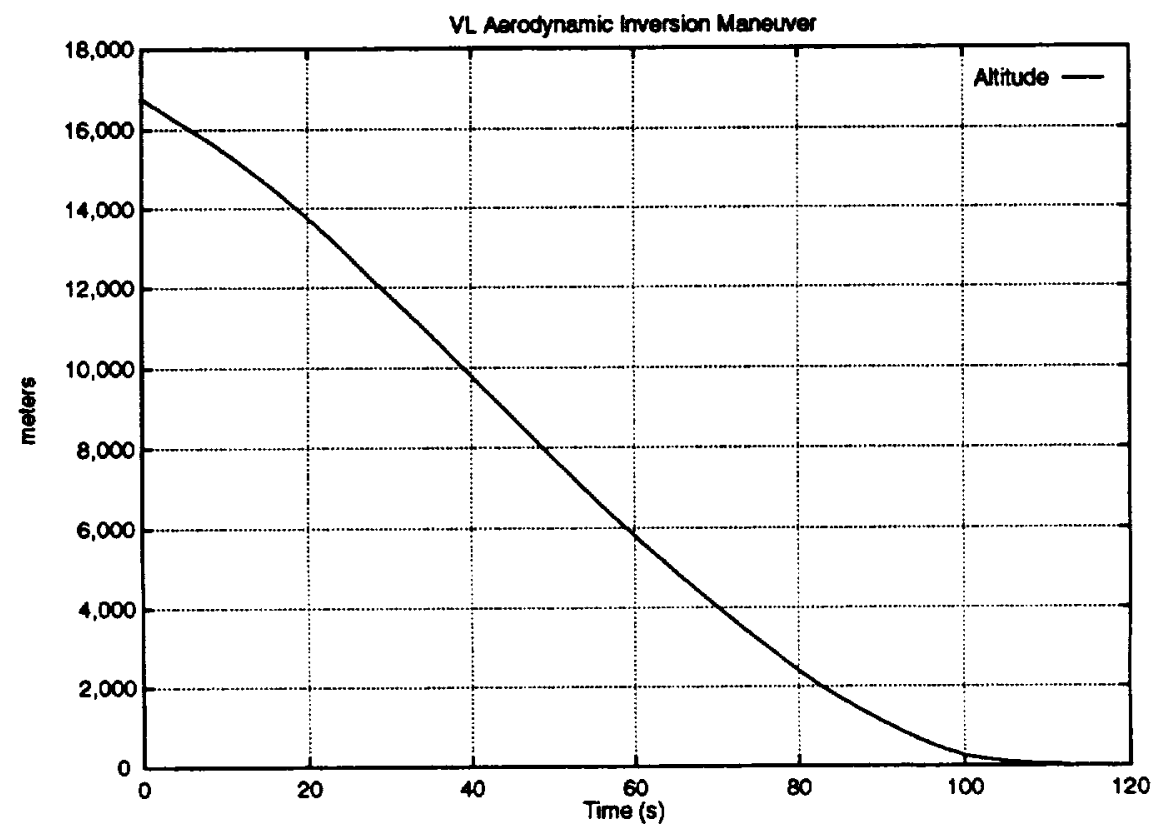

Figure 7. VL aerodynamic inversion maneuver, altitude versus time.

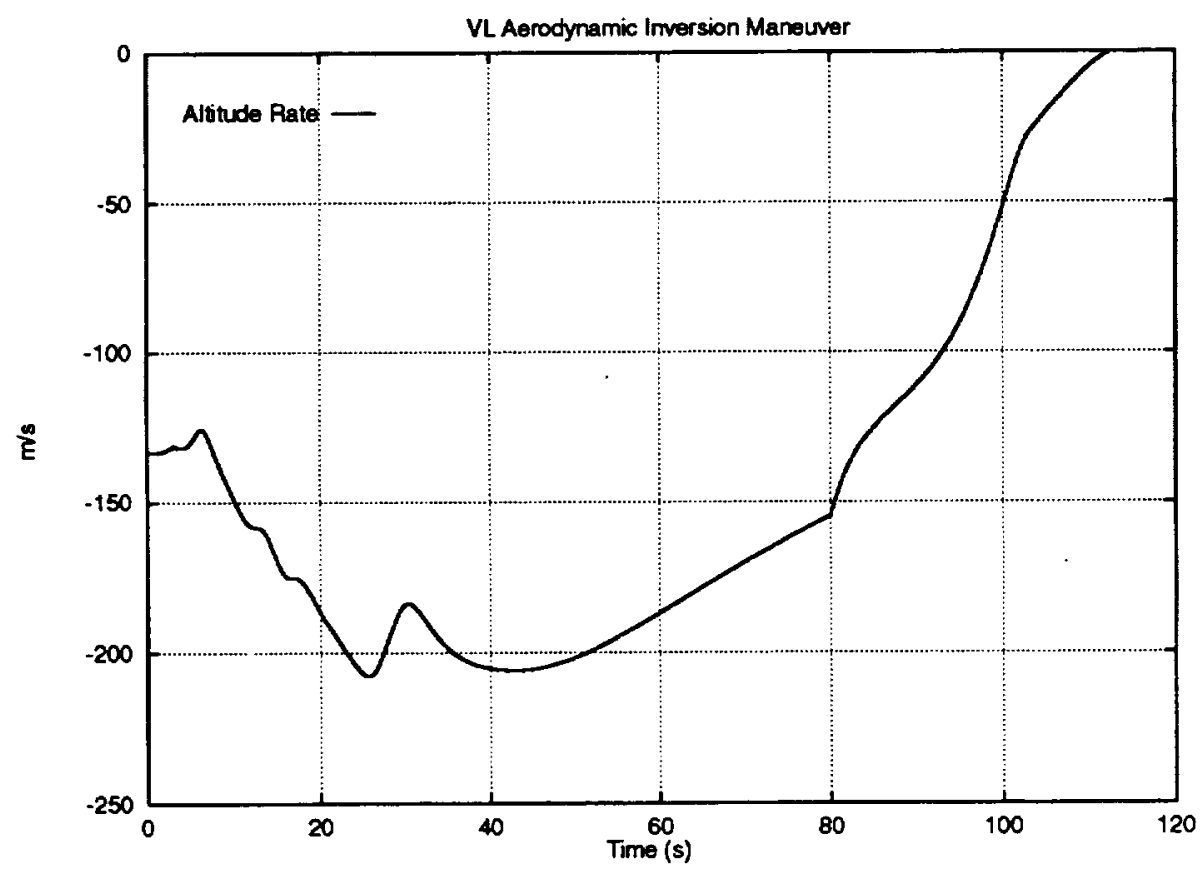

Figure 8. VL aerodynamic inversion maneuver, altitude rate versus time. 


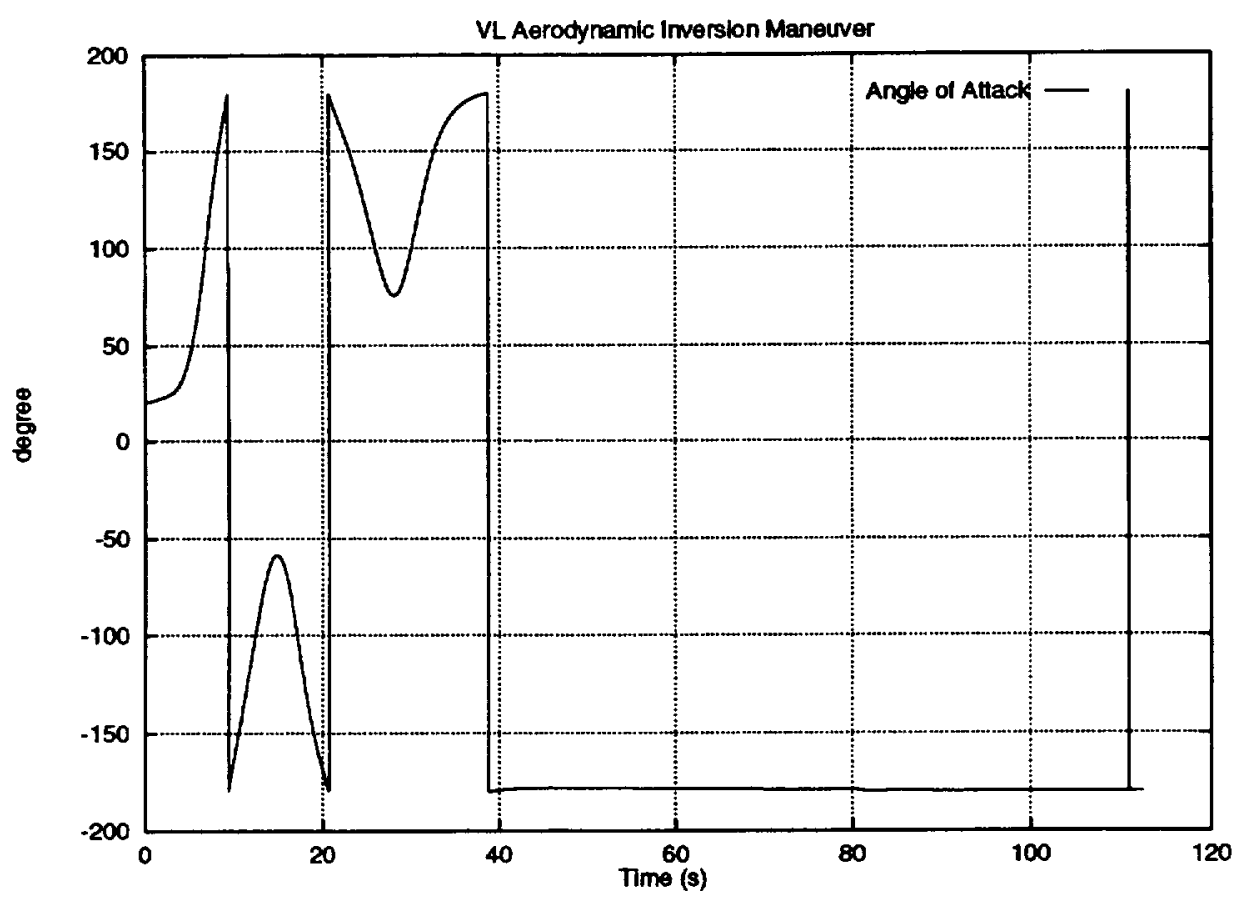

Figure 9. VL aerodynamic inversion maneuver, angle of attack versus time.

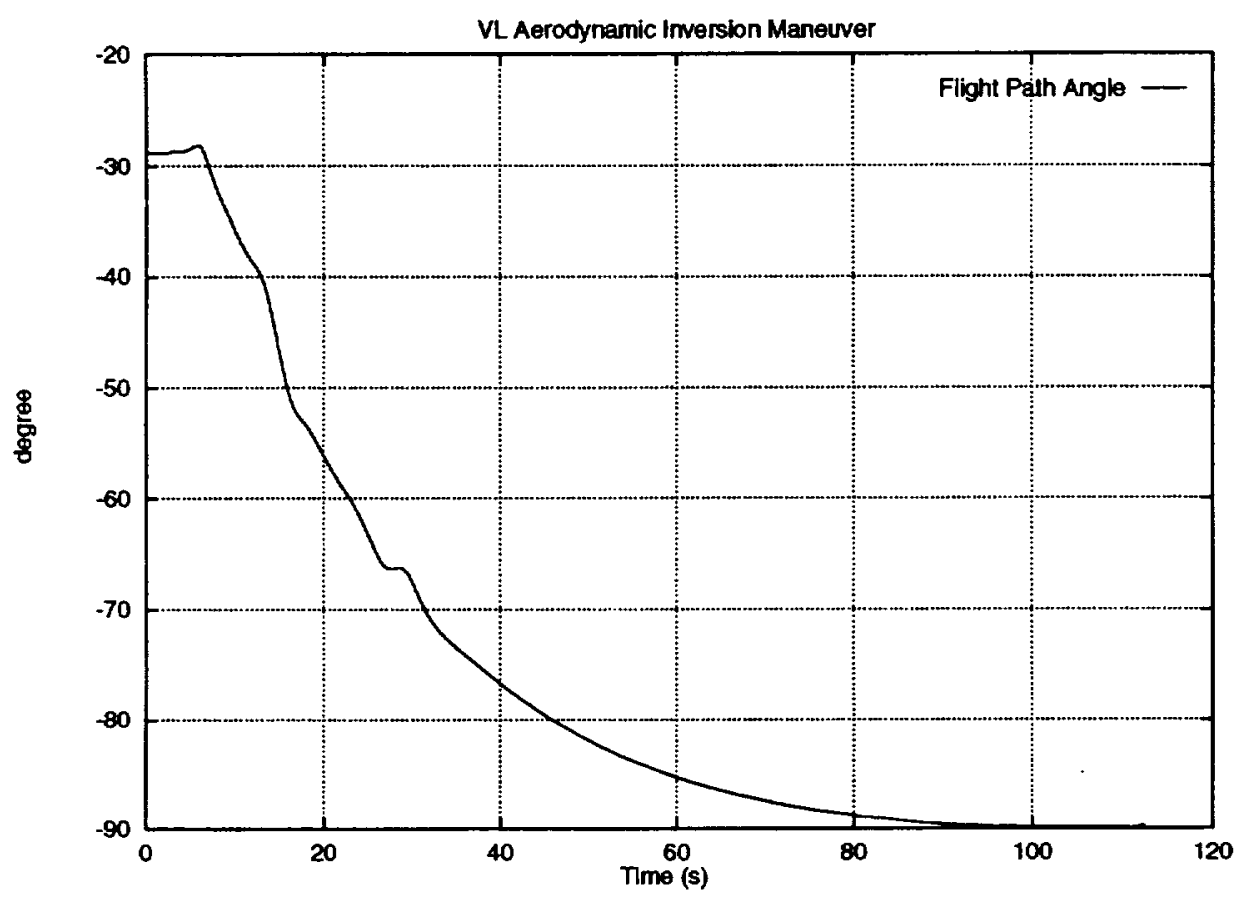

Figure 10. VL aerodynamic inversion maneuver, flight path angle versus time. 


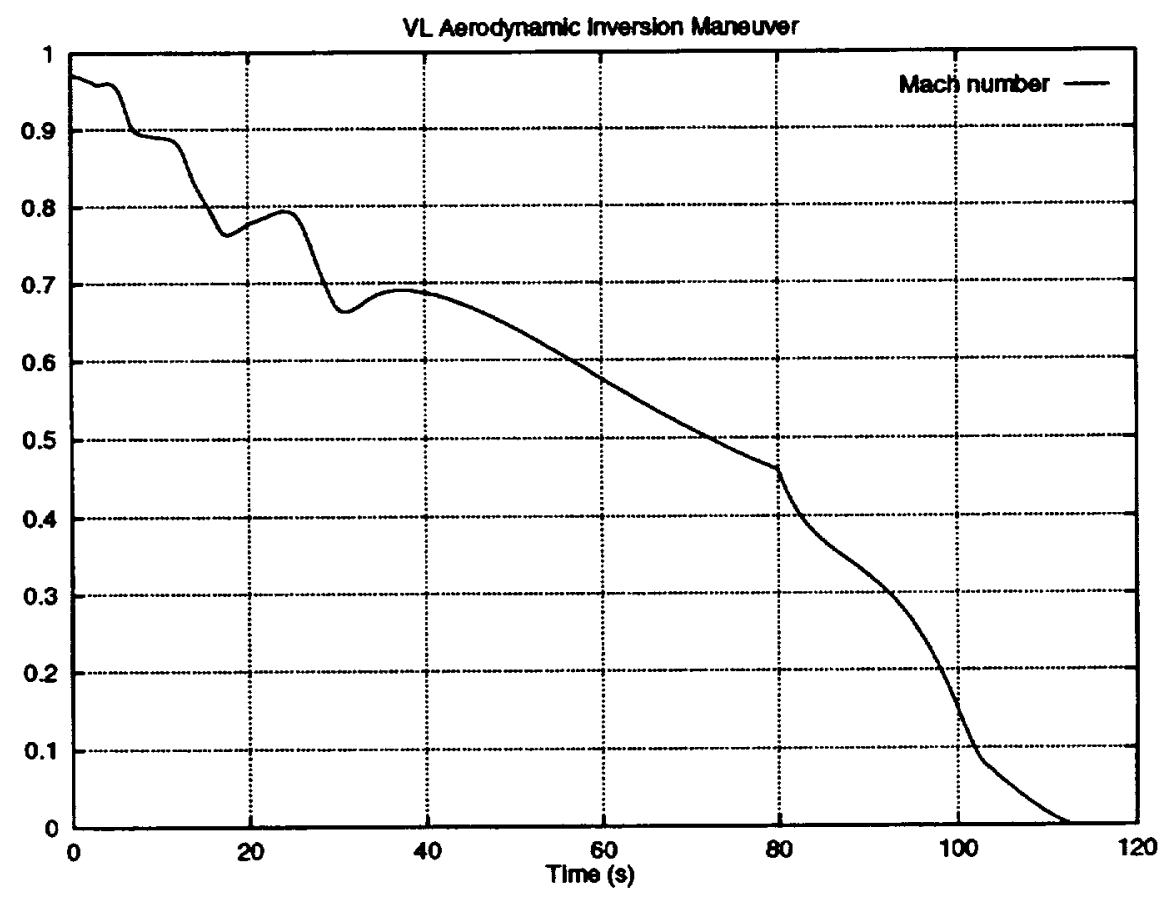

Figure 11. VL aerodynamic inversion maneuver, Mach number versus time.

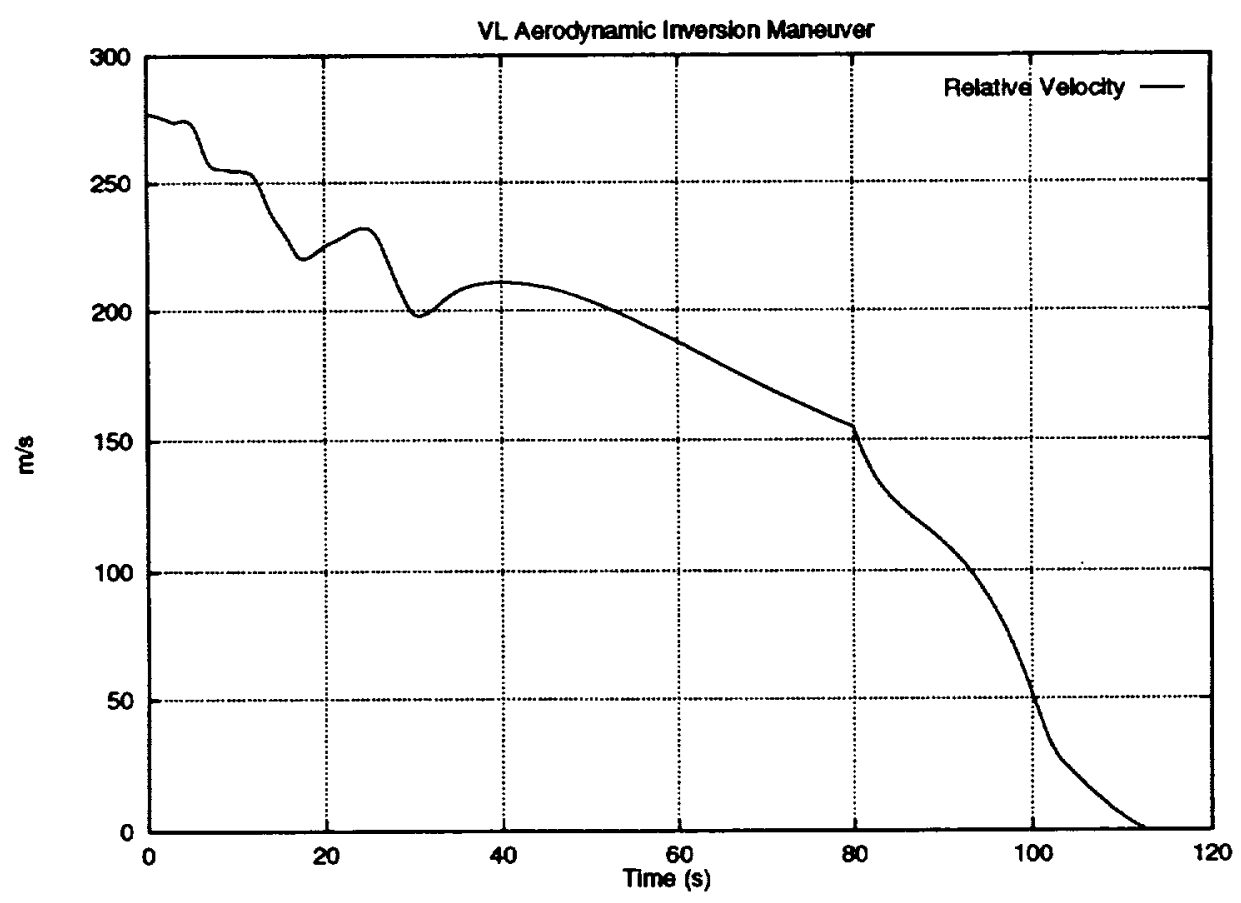

Figure 12. VL aerodynamic inversion maneuver, relative velocity versus time. 


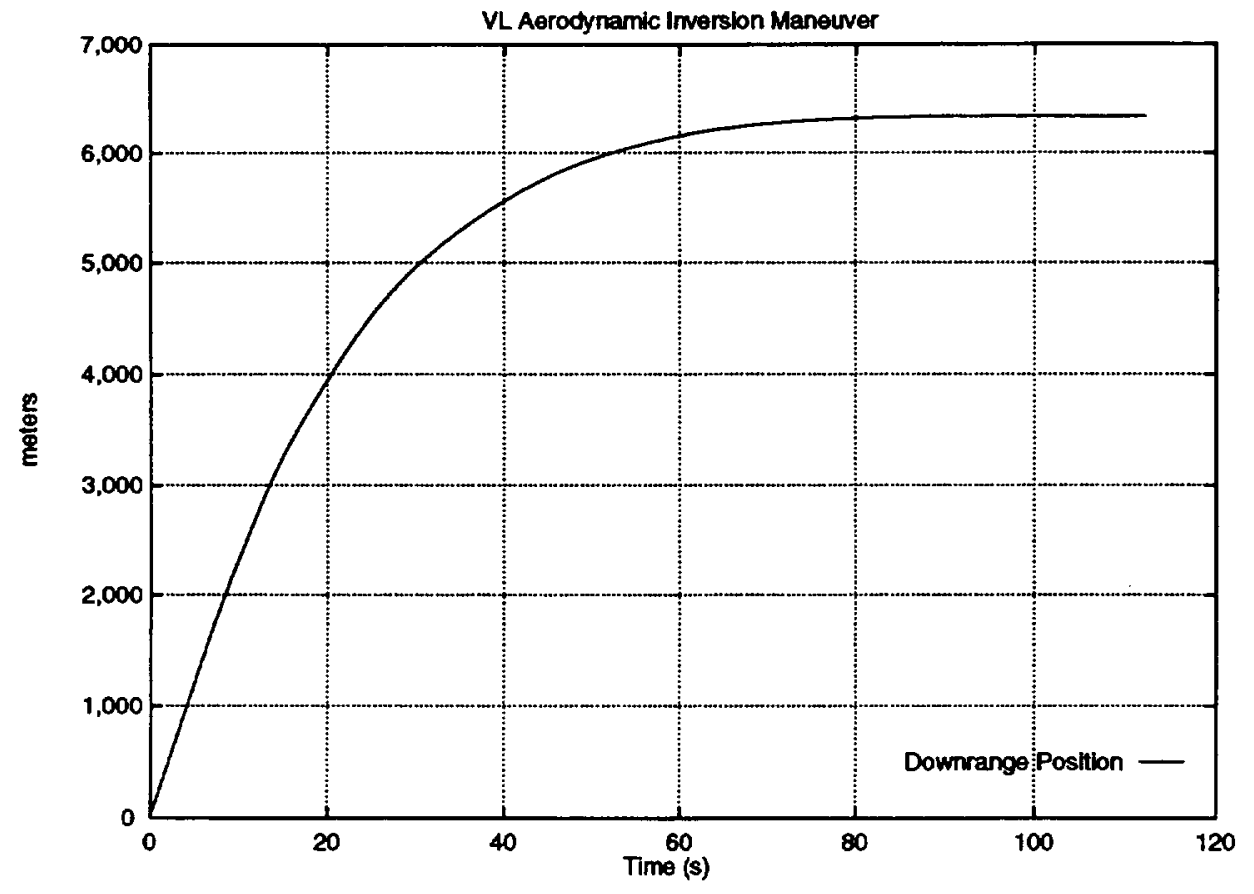

Figure 13. VL aerodynamic inversion maneuver, downrange position versus time.

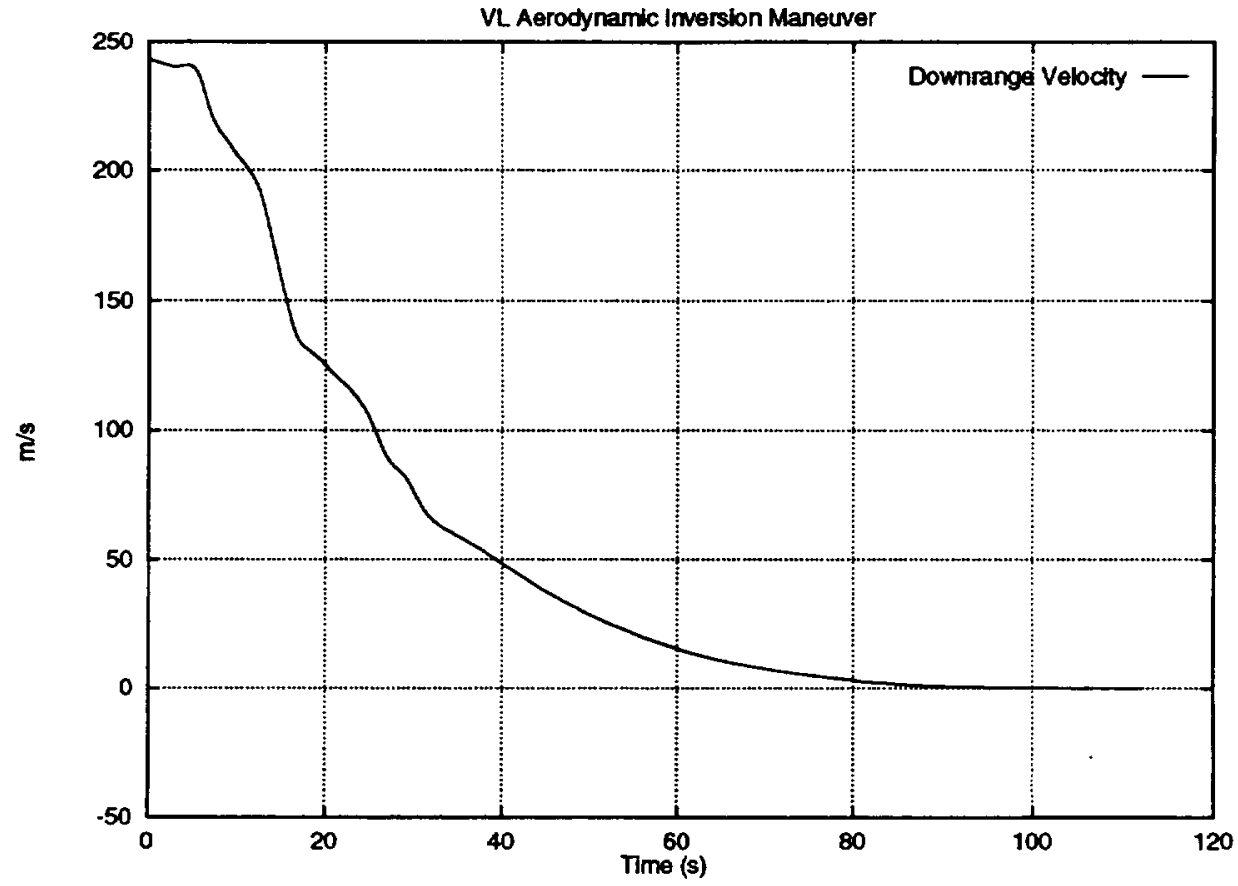

Figure 14. VL aerodynamic inversion maneuver, downrange velocity versus time. 


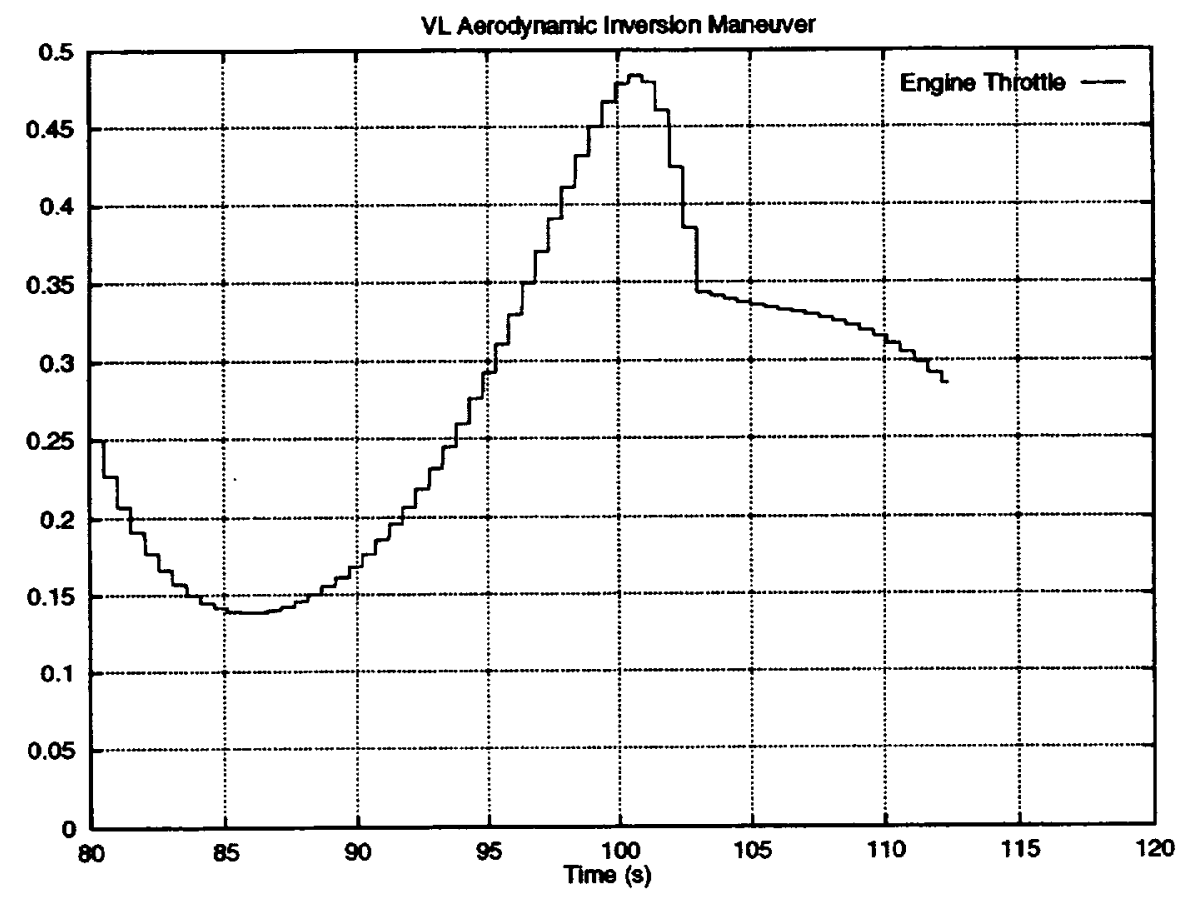

Figure 15. VL aerodynamic inversion maneuver, engine throttle versus time.

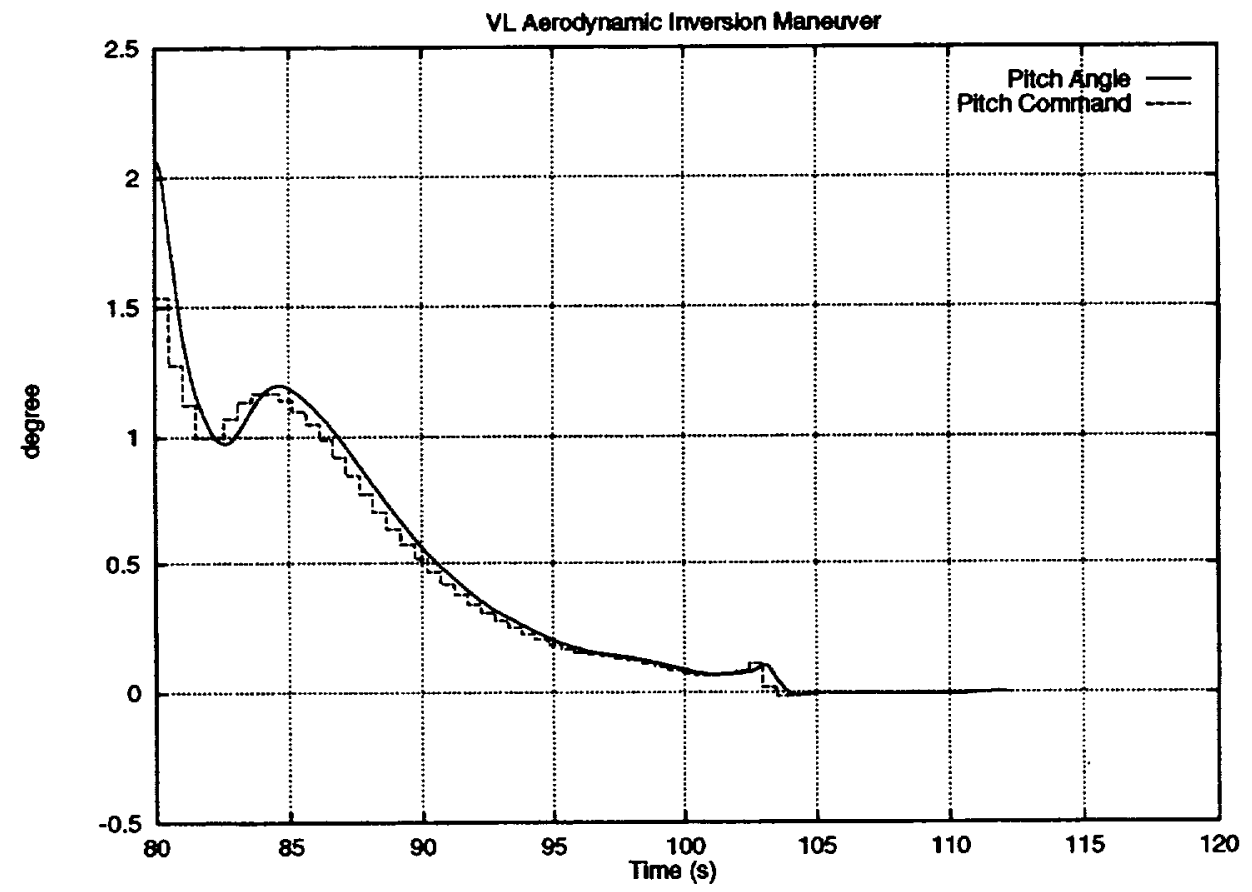

Figure 16. VL aerodynamic inversion maneuver, pitch angle and command versus time. 


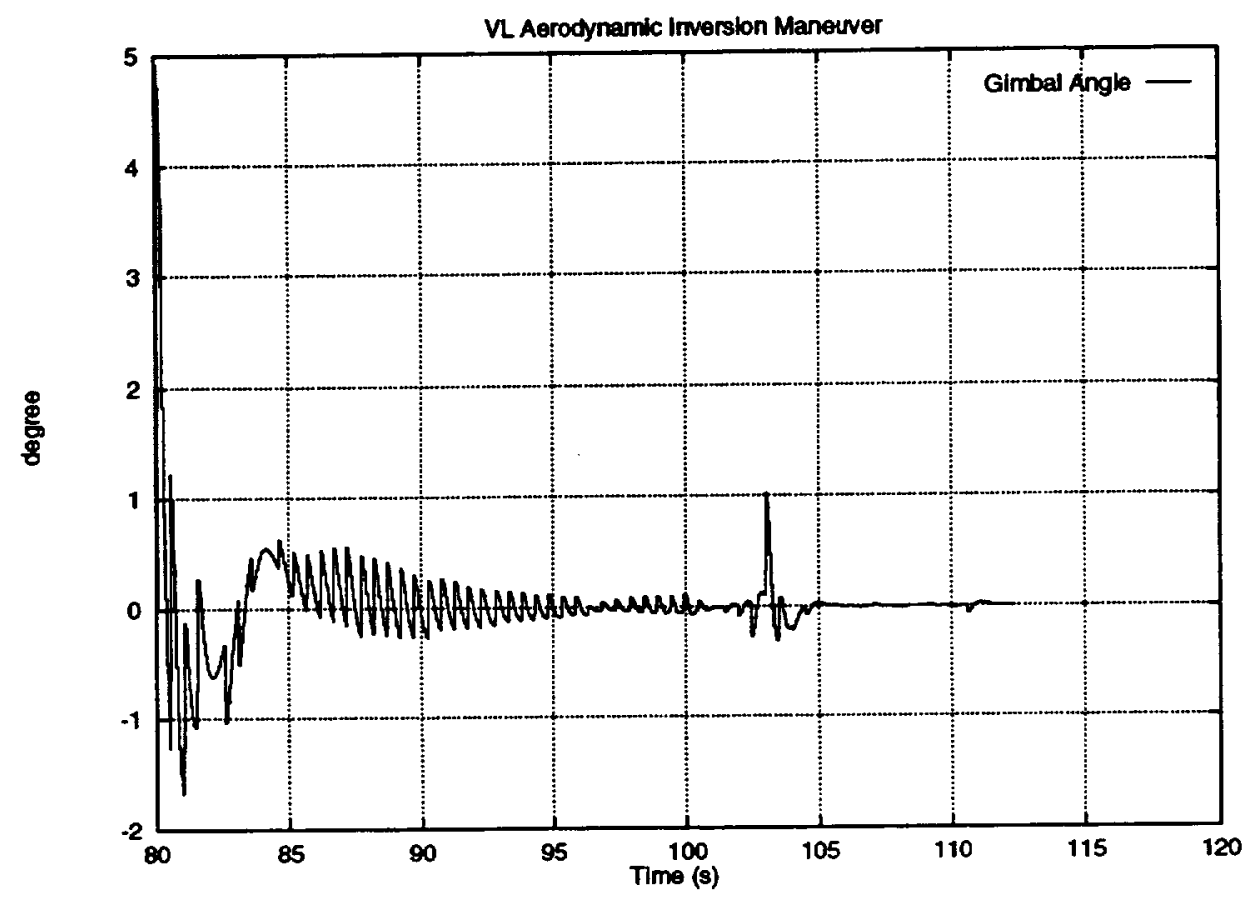

Figure 17. VL aerodynamic inversion maneuver, gimbal angle versus time.

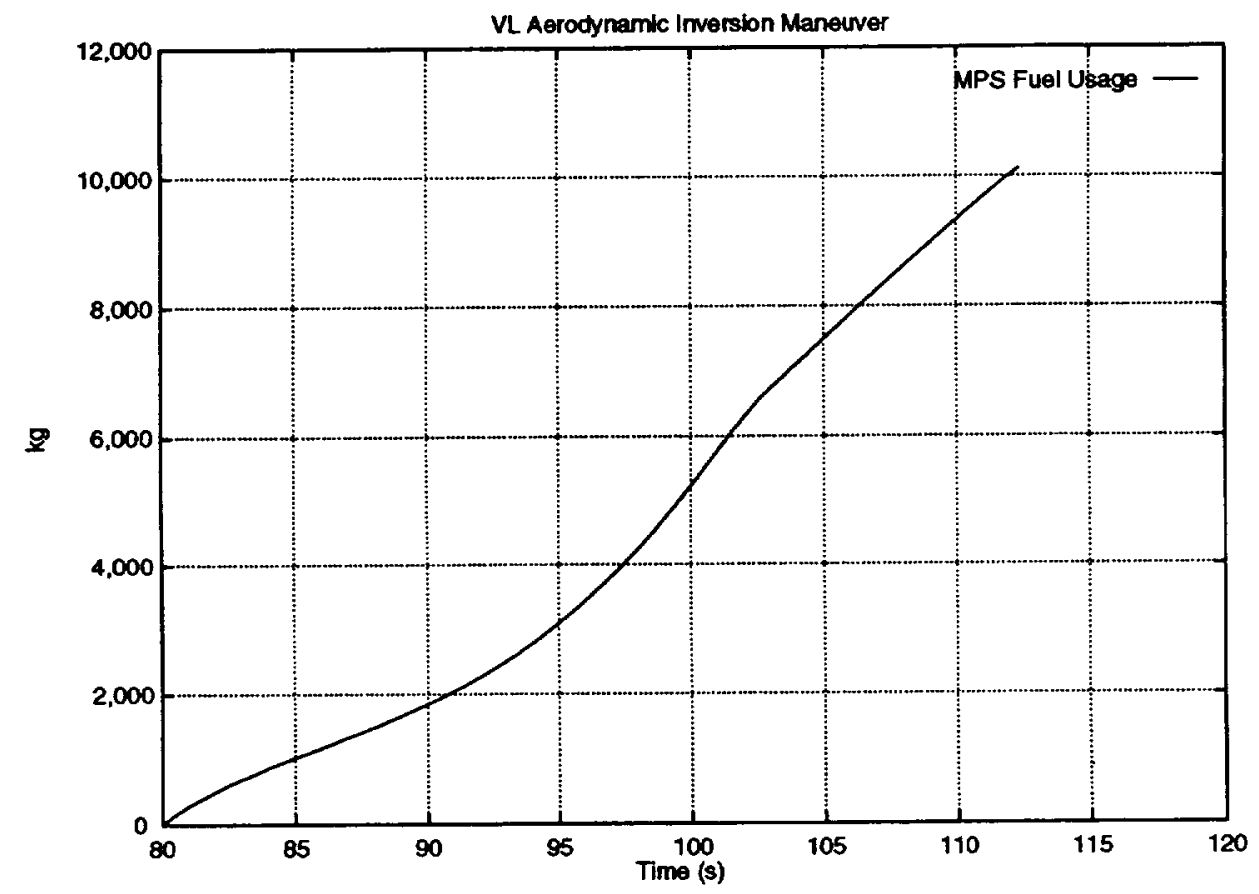

Figure 18. VL aerodynamic inversion maneuver, main propulsion system propellant usage versus time. 


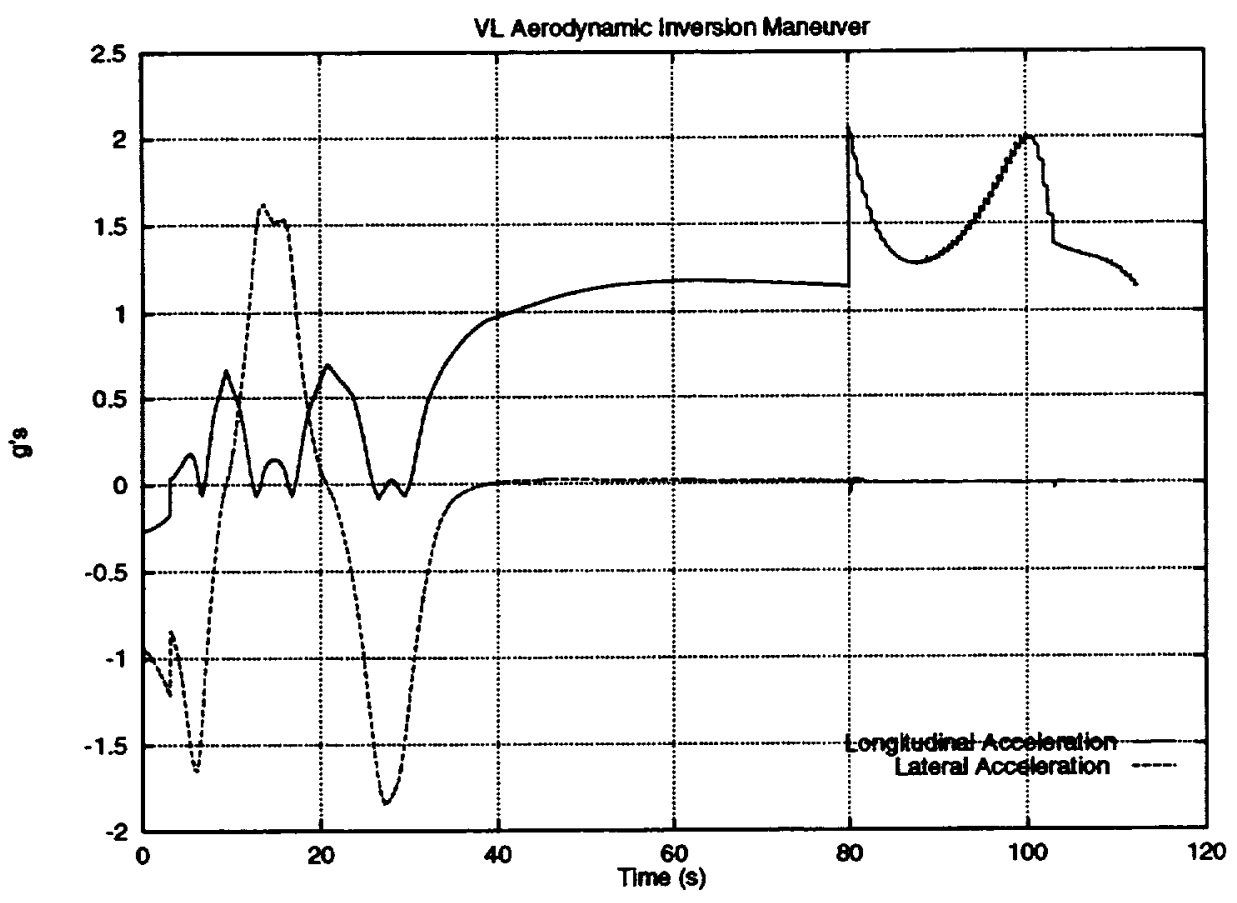

Figure 19. VL aerodynamic inversion maneuver, vehicle accelerations versus time.

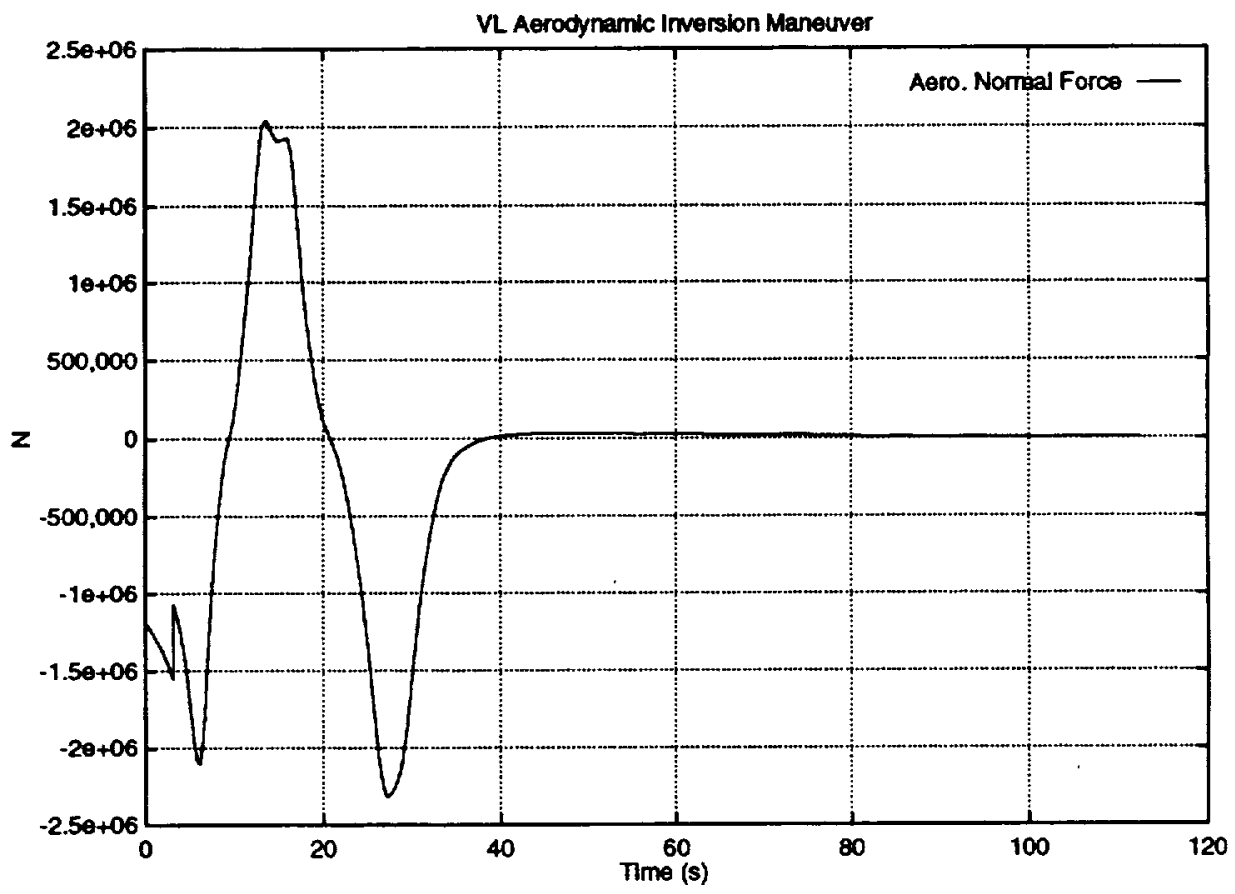

Figure 20. VL aerodynamic inversion maneuver, aerodynamic normal force versus time. 


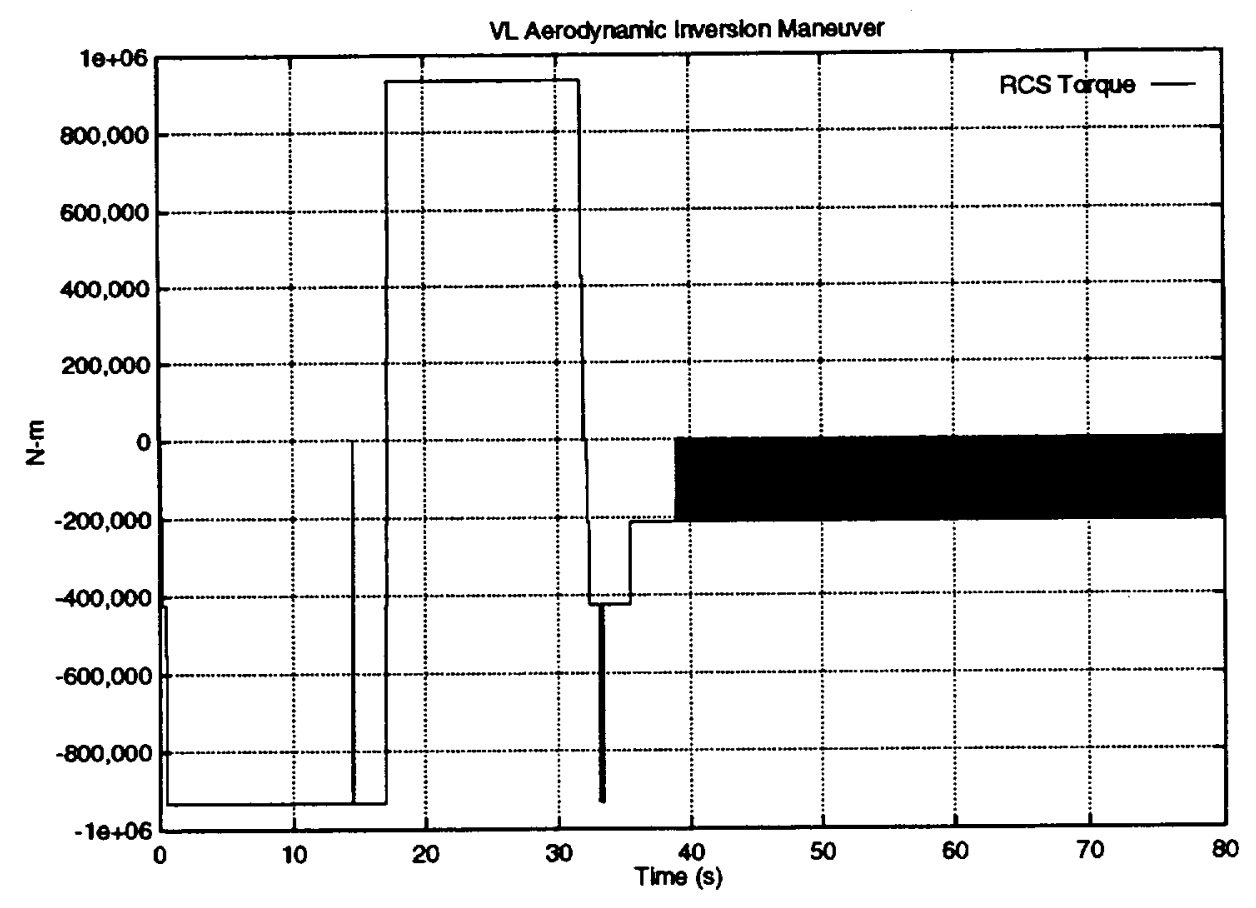

Figure 21. VL aerodynamic inversion maneuver, RCS torque versus time.

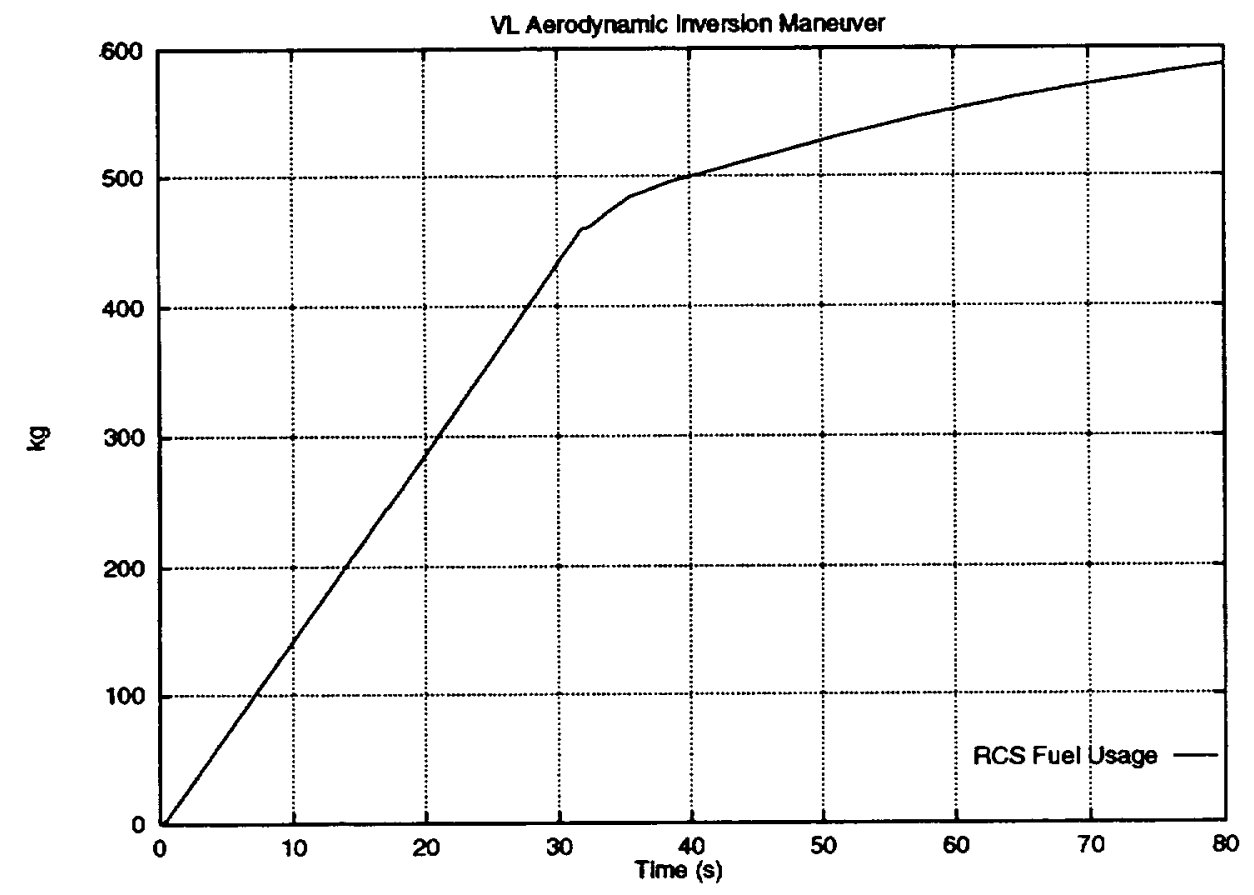

Figure 22. VL aerodynamic inversion maneuver, RCS propellant usage versus time. 


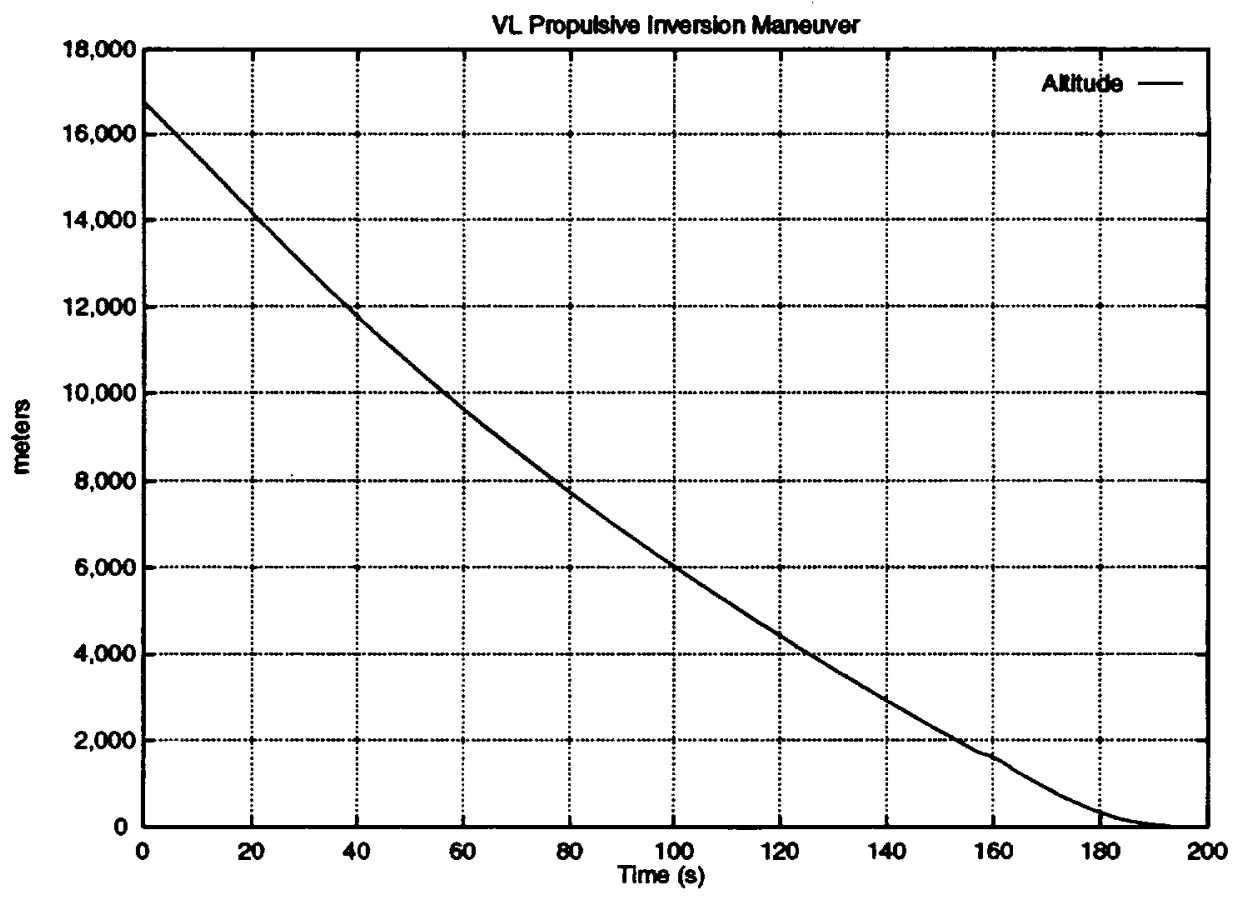

Figure 23. VL propulsive inversion maneuver, altitude versus time.

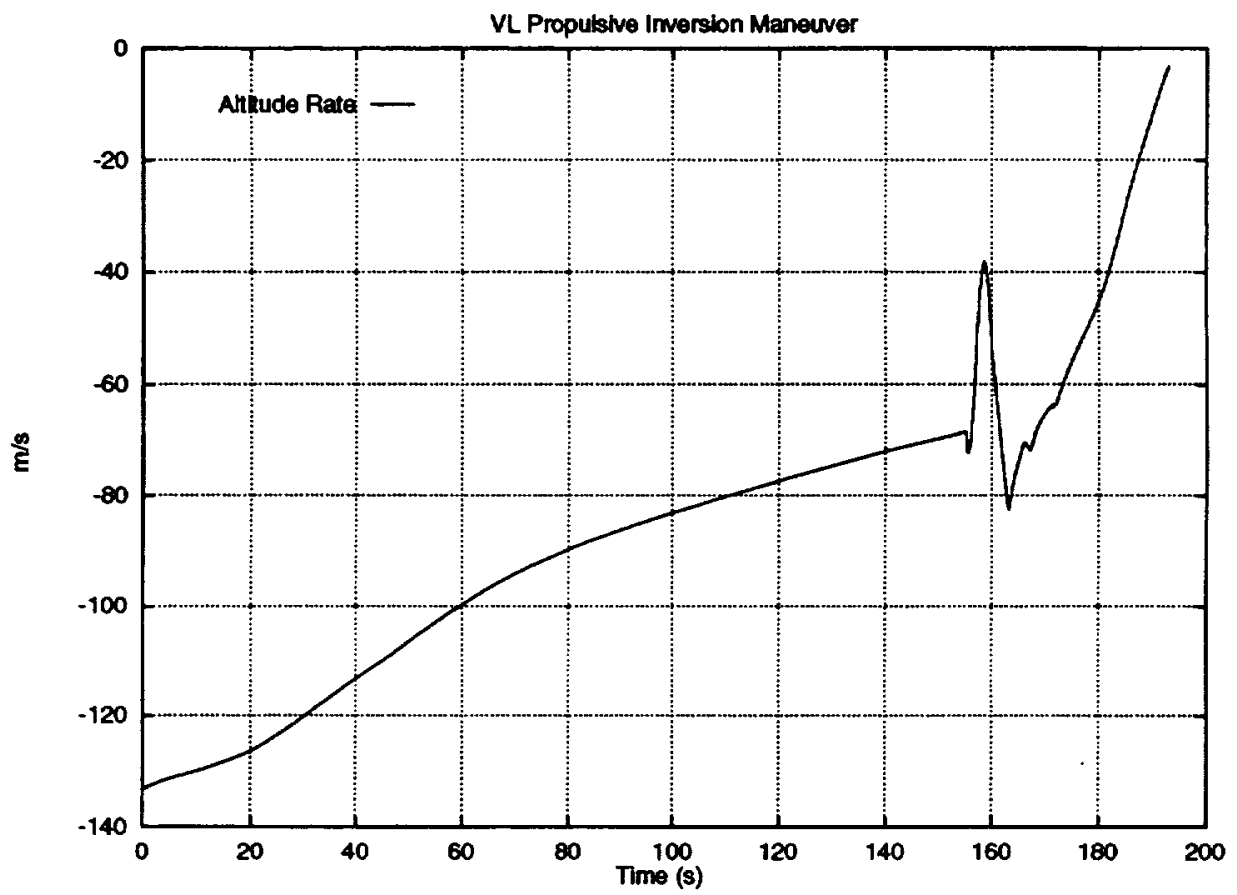

Figure 24. VL propulsive inversion maneuver, altitude rate versus time. 


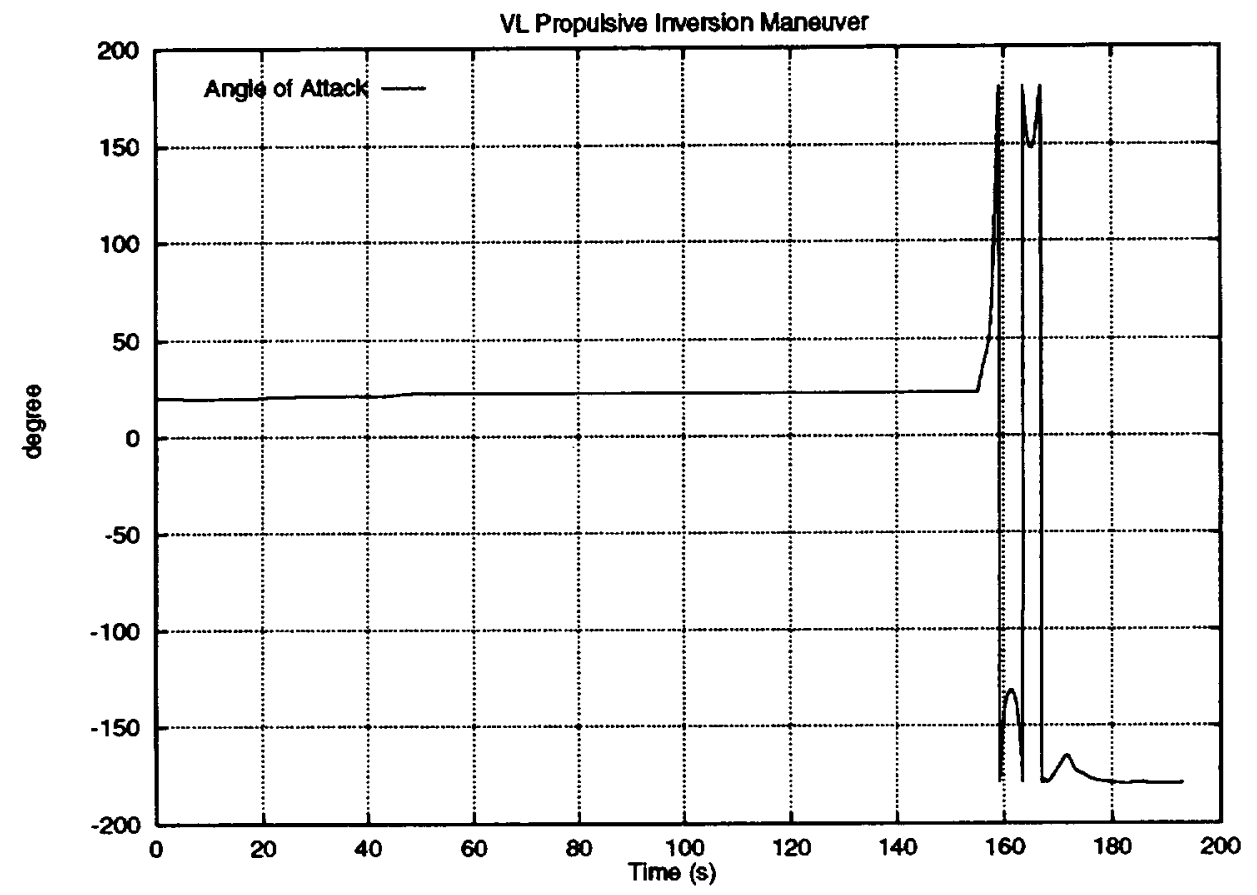

Figure 25. VL propulsive inversion maneuver, angle of attack versus time.

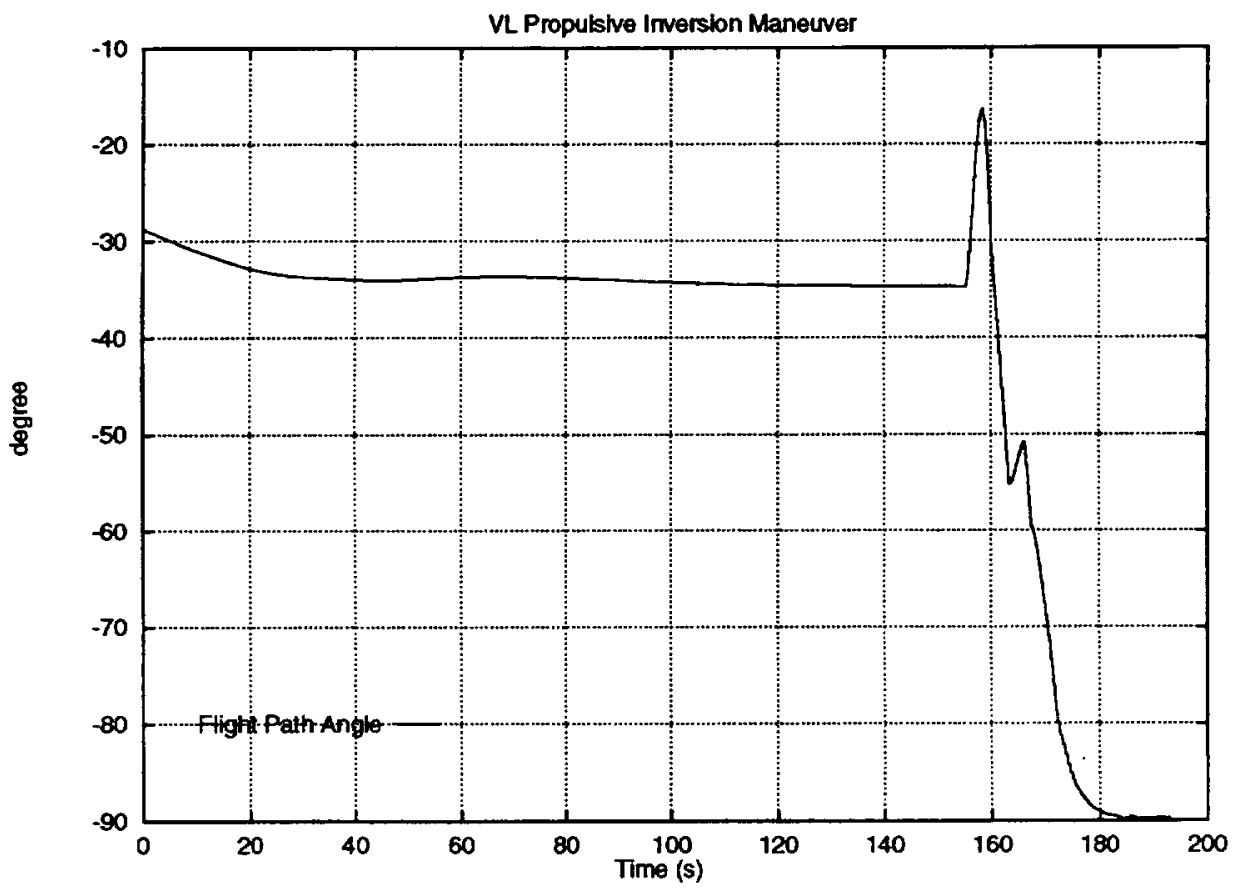

Figure 26. VL propulsive inversion maneuver, flight path angle versus time. 


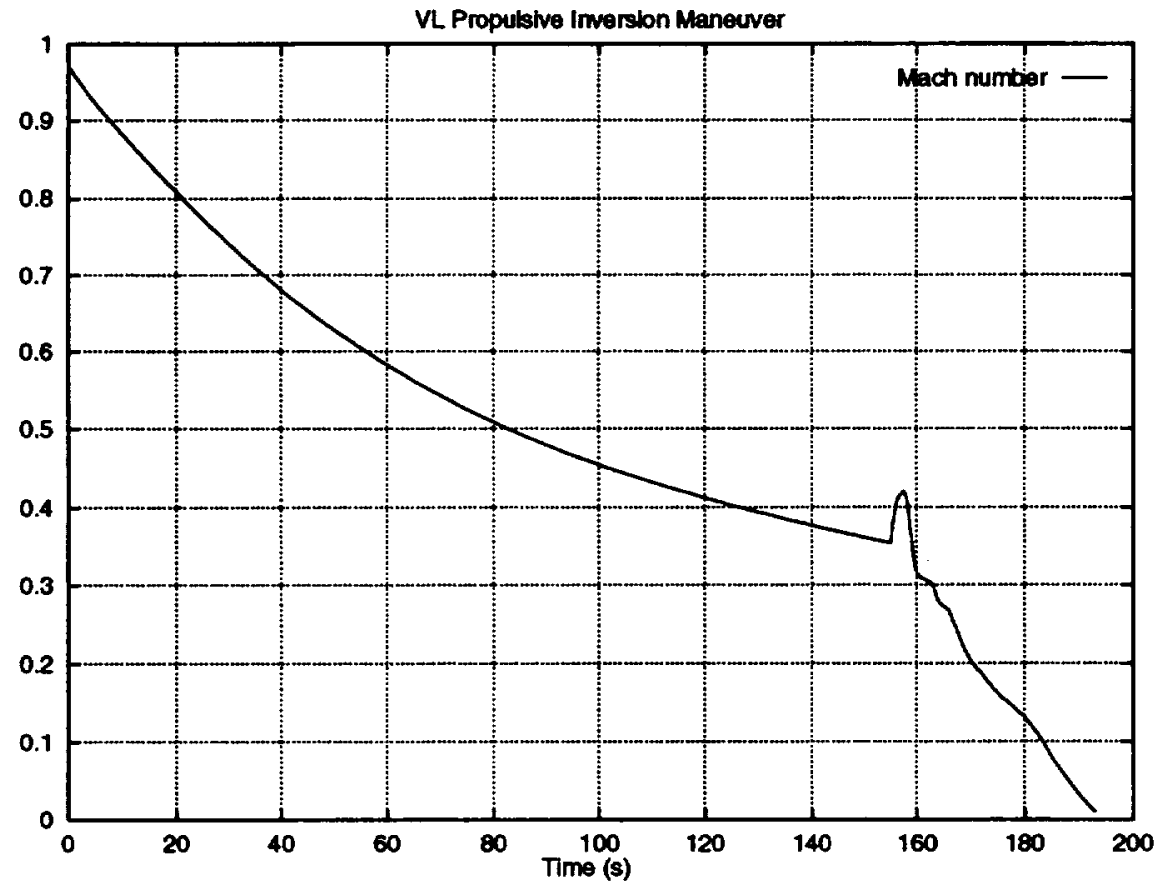

Figure 27. VL propulsive inversion maneuver, Mach number versus time.

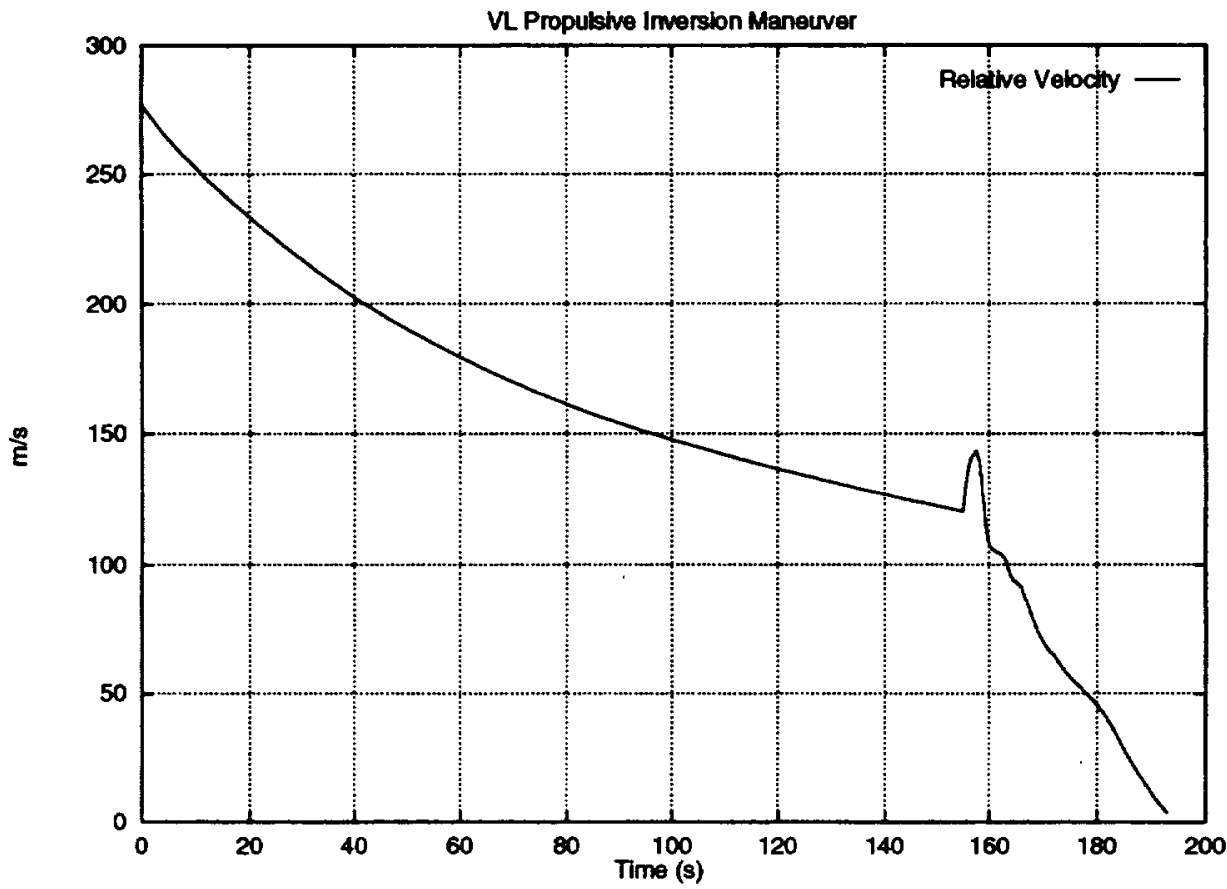

Figure 28. VL propulsive inversion maneuver, relative velocity versus time. 


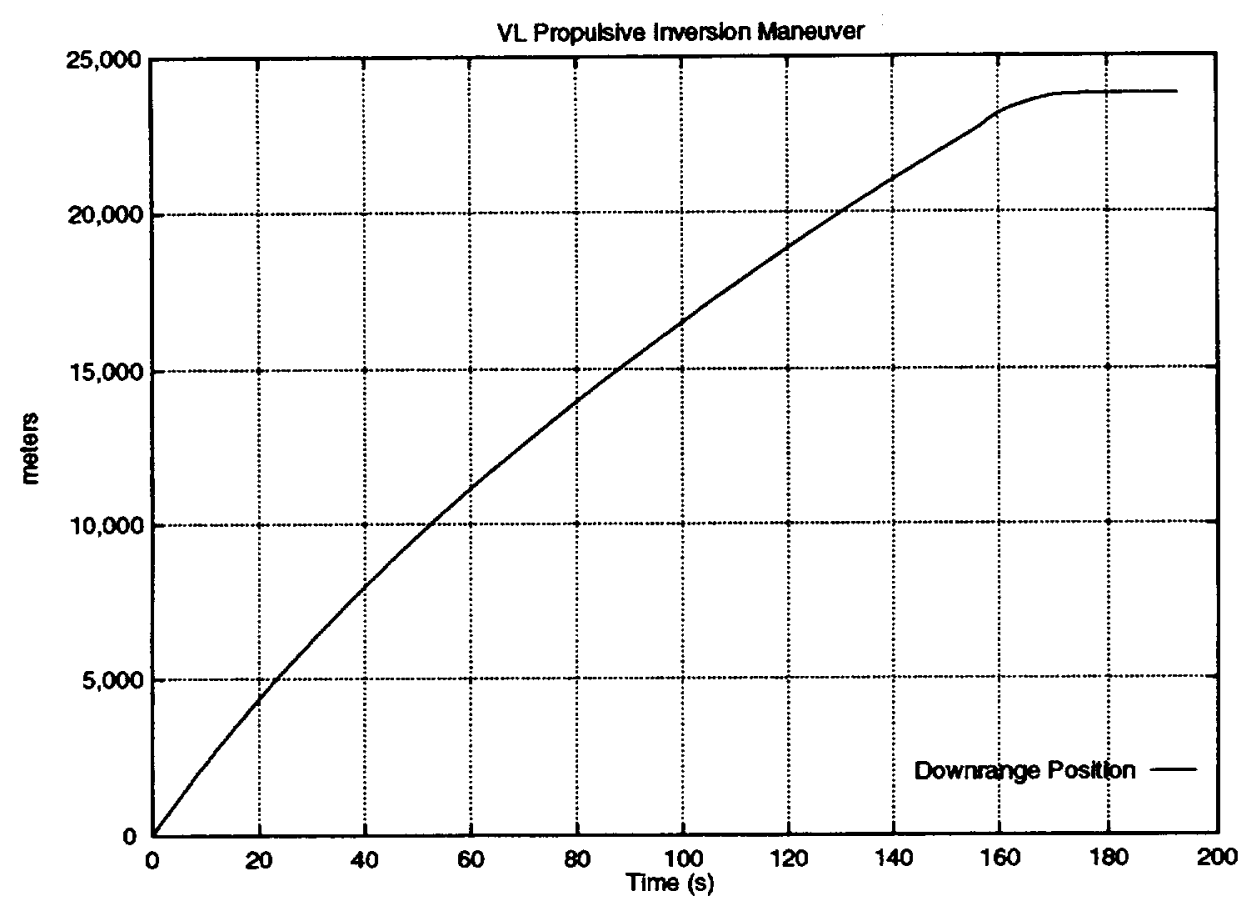

Figure 29. VL propulsive inversion maneuver, downrange position versus time.

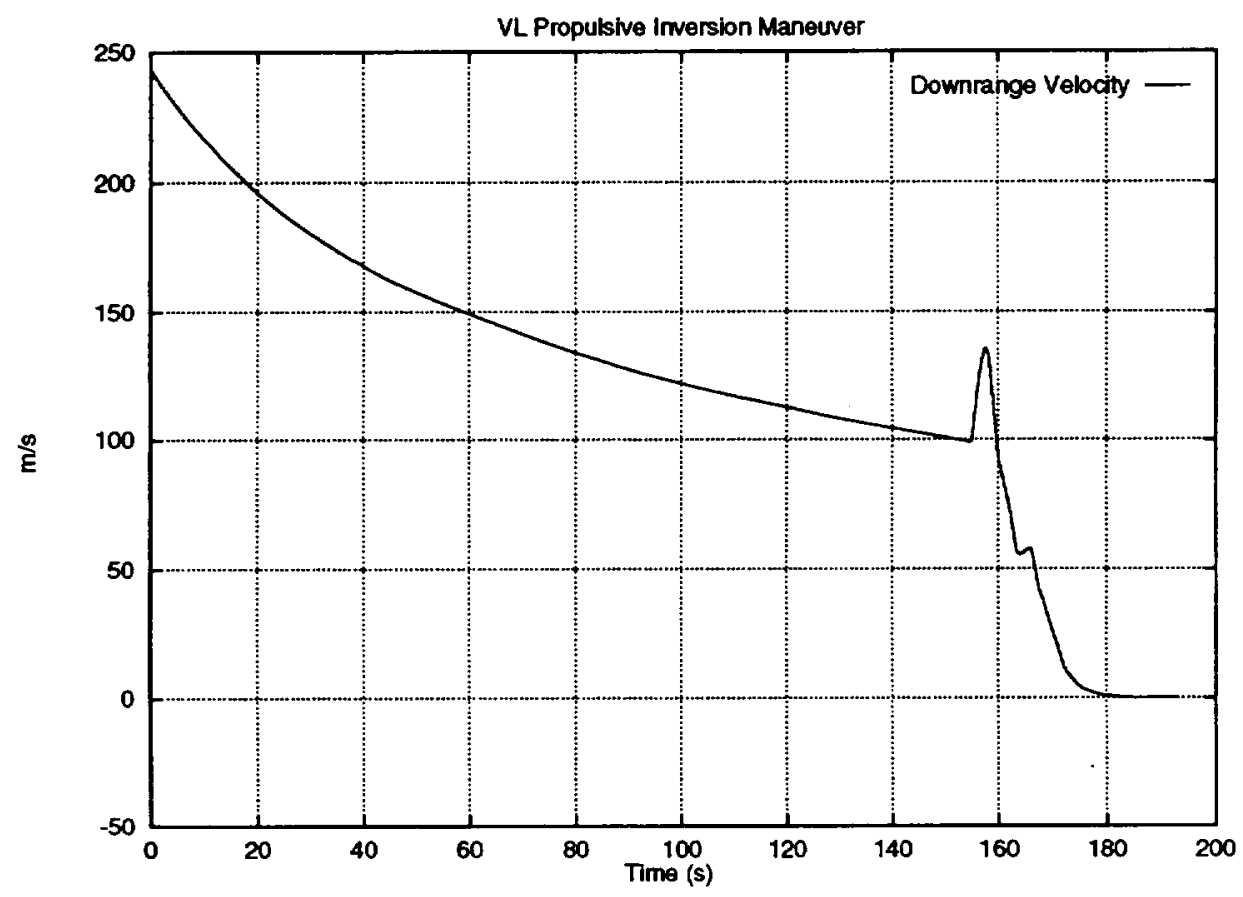

Figure 30. VL propulsive inversion maneuver, downrange velocity versus time. 


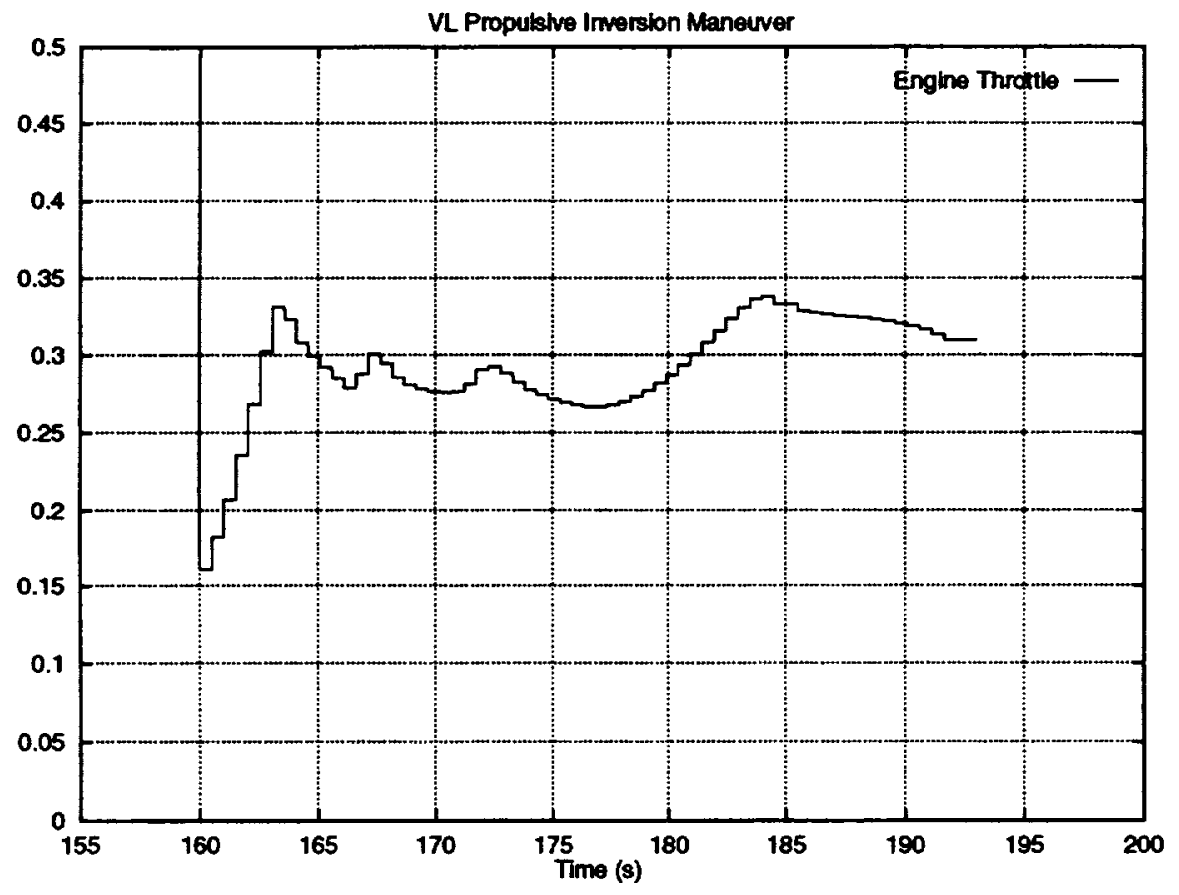

Figure 31. VL propulsive inversion maneuver, engine throttle versus time.

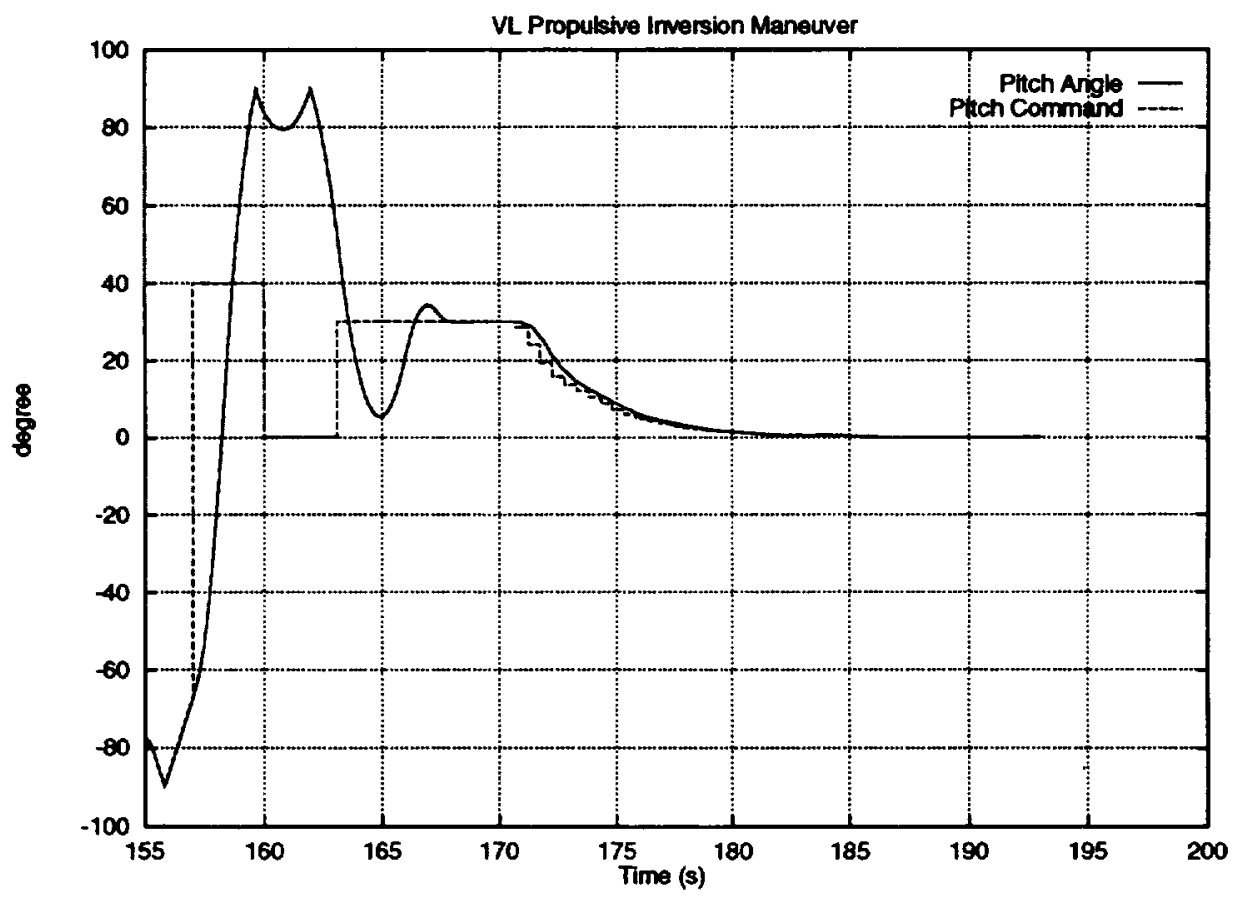

Figure 32. VL propulsive inversion maneuver, pitch angle and command versus time. 


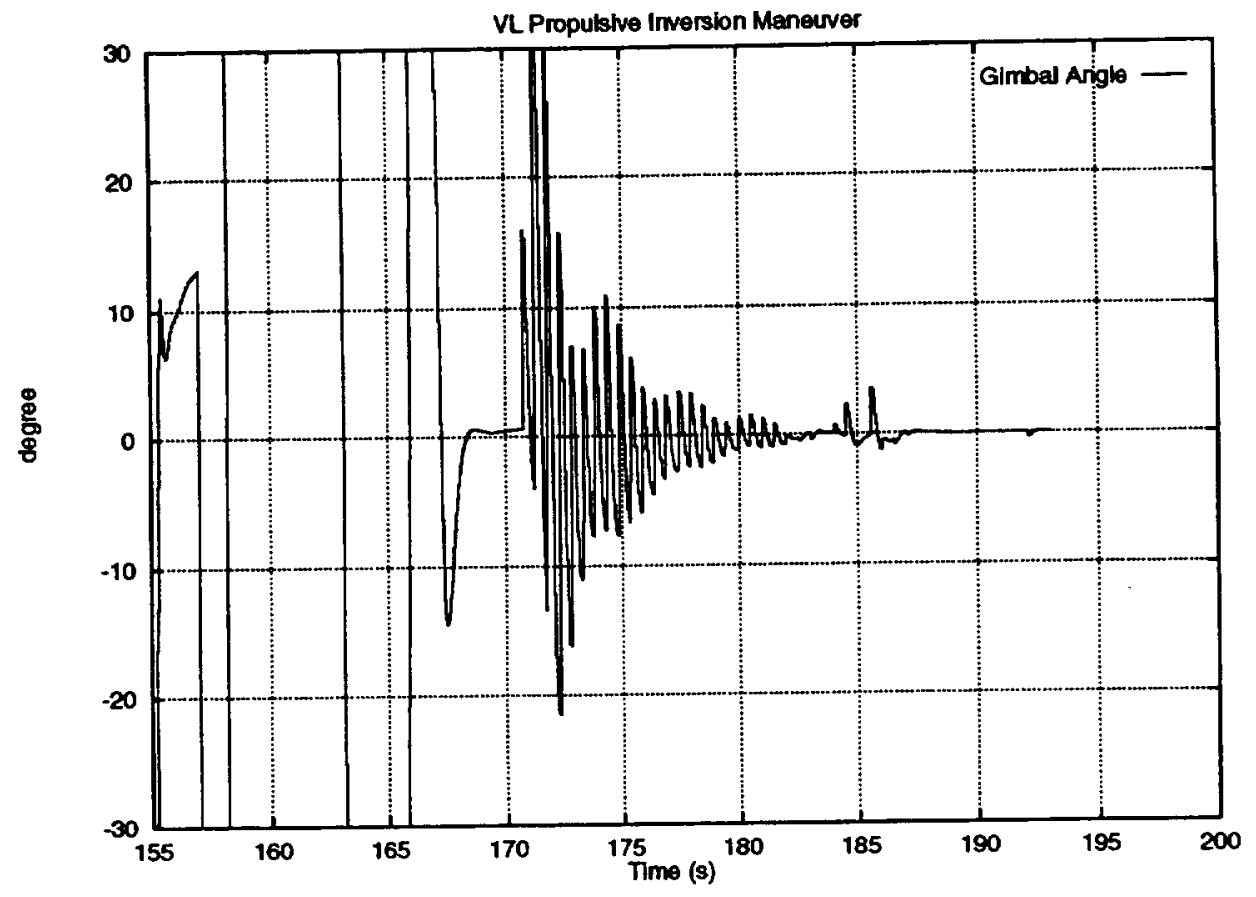

Figure 33. VL propulsive inversion maneuver, gimbal angle versus time.

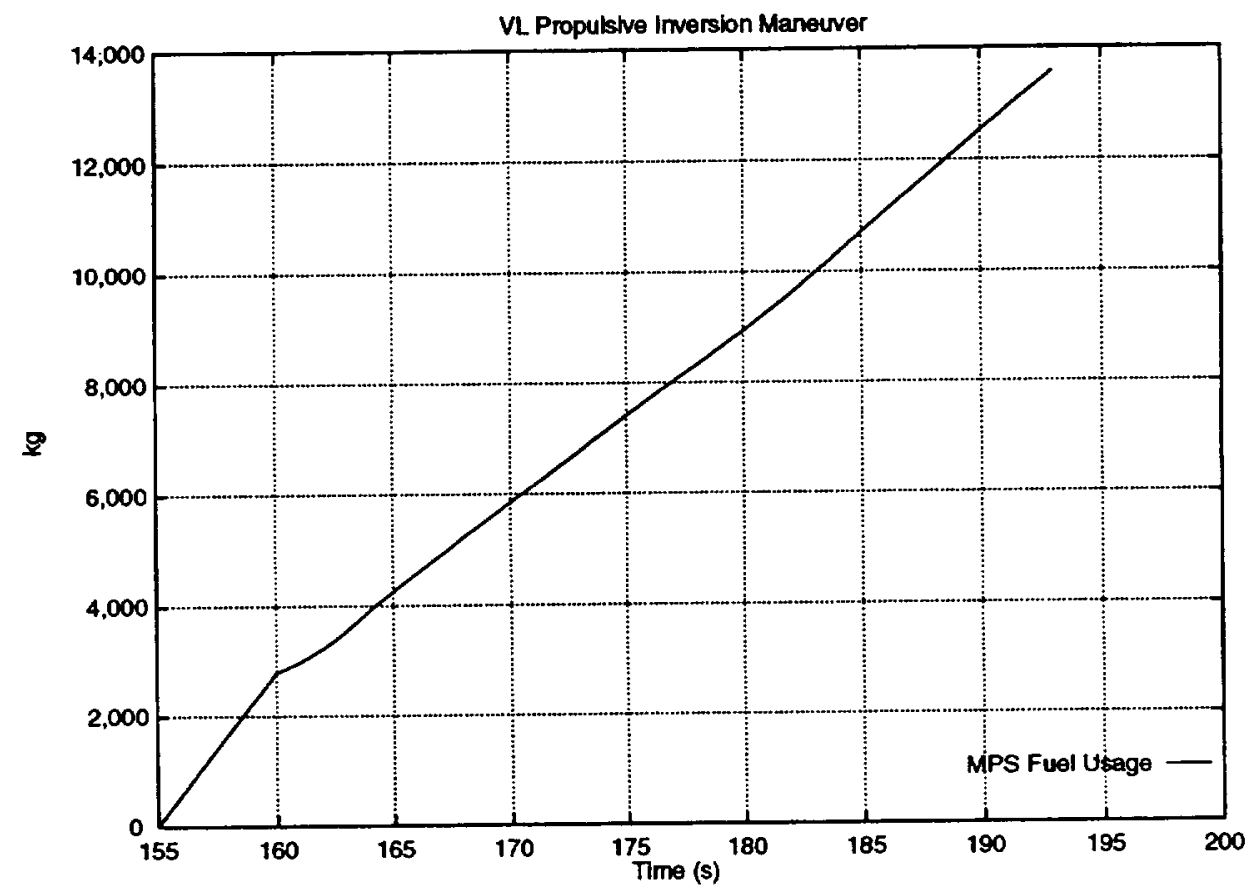

Figure 34. VL propulsive inversion maneuver, main propulsion system propellant usage versus time. 


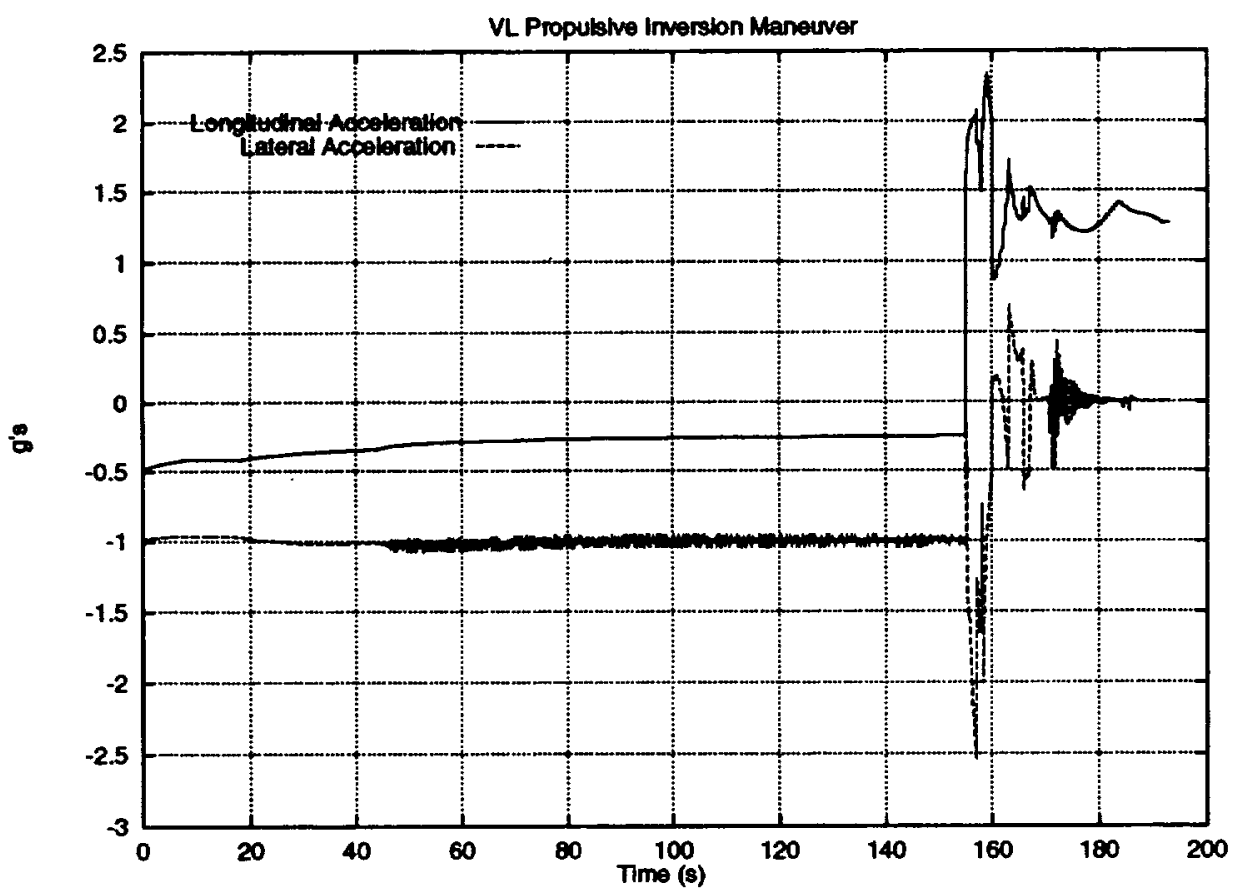

Figure 35. VL propulsive inversion maneuver, vehicle accelerations versus time.

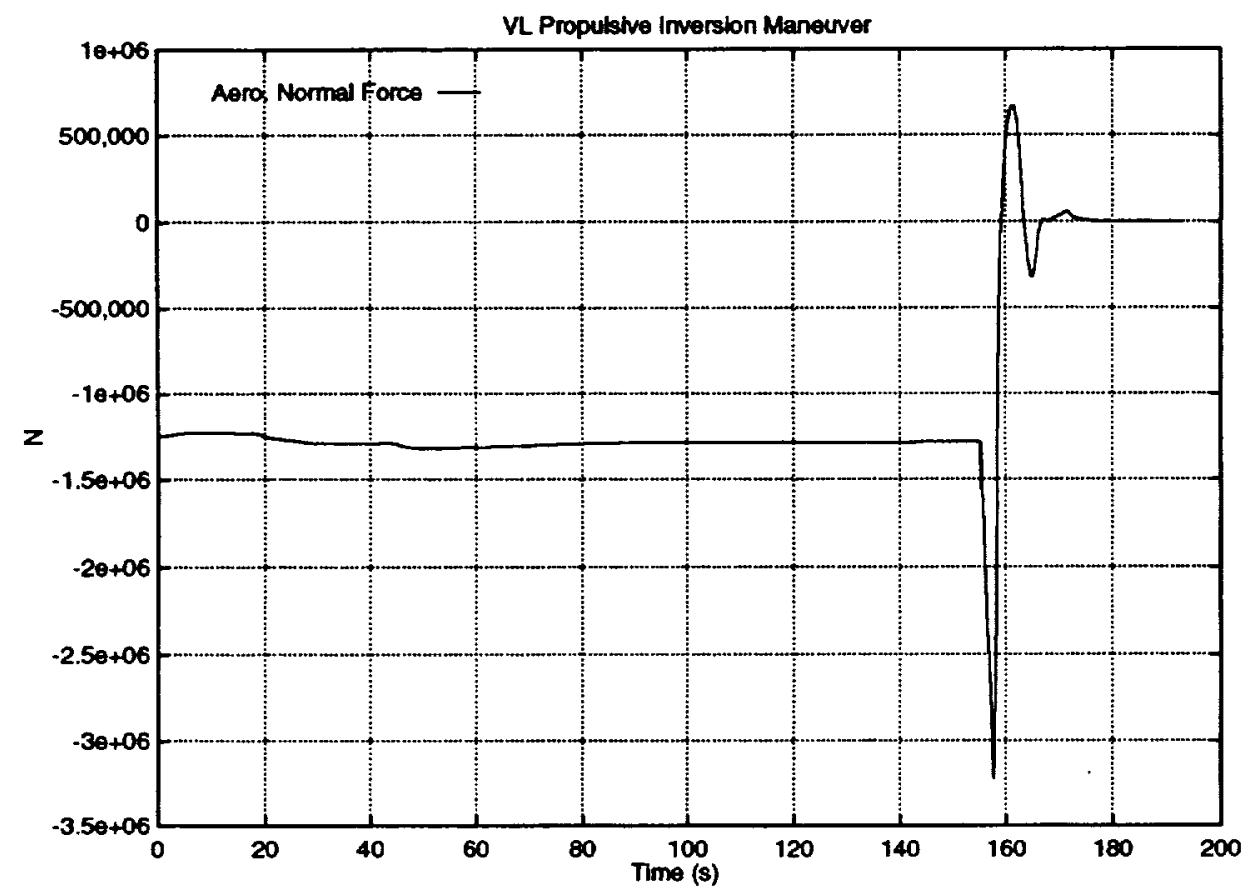

Figure 36. VL propulsive inversion maneuver, aerodynamic normal force versus time. 


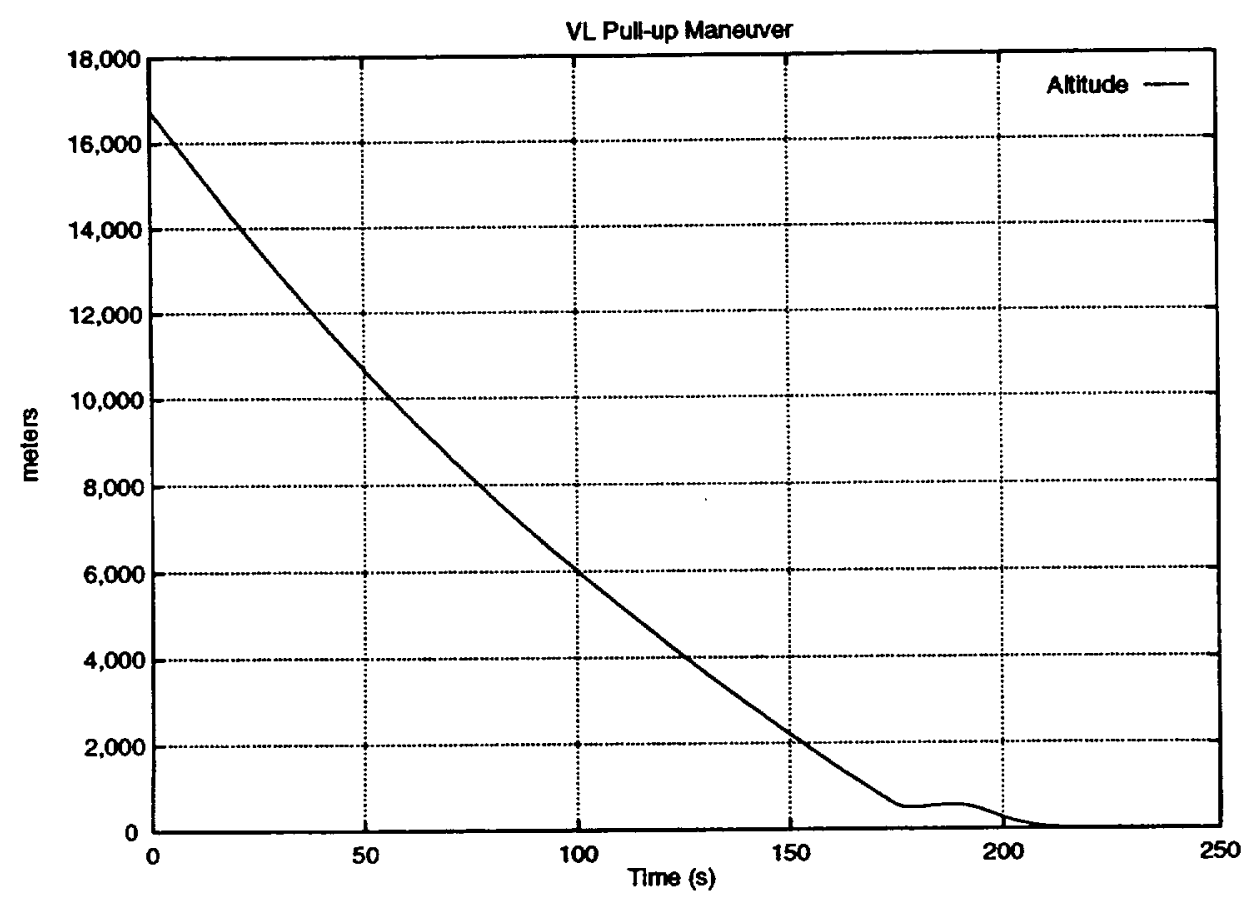

Figure 37. VL pullup maneuver, altitude versus time.

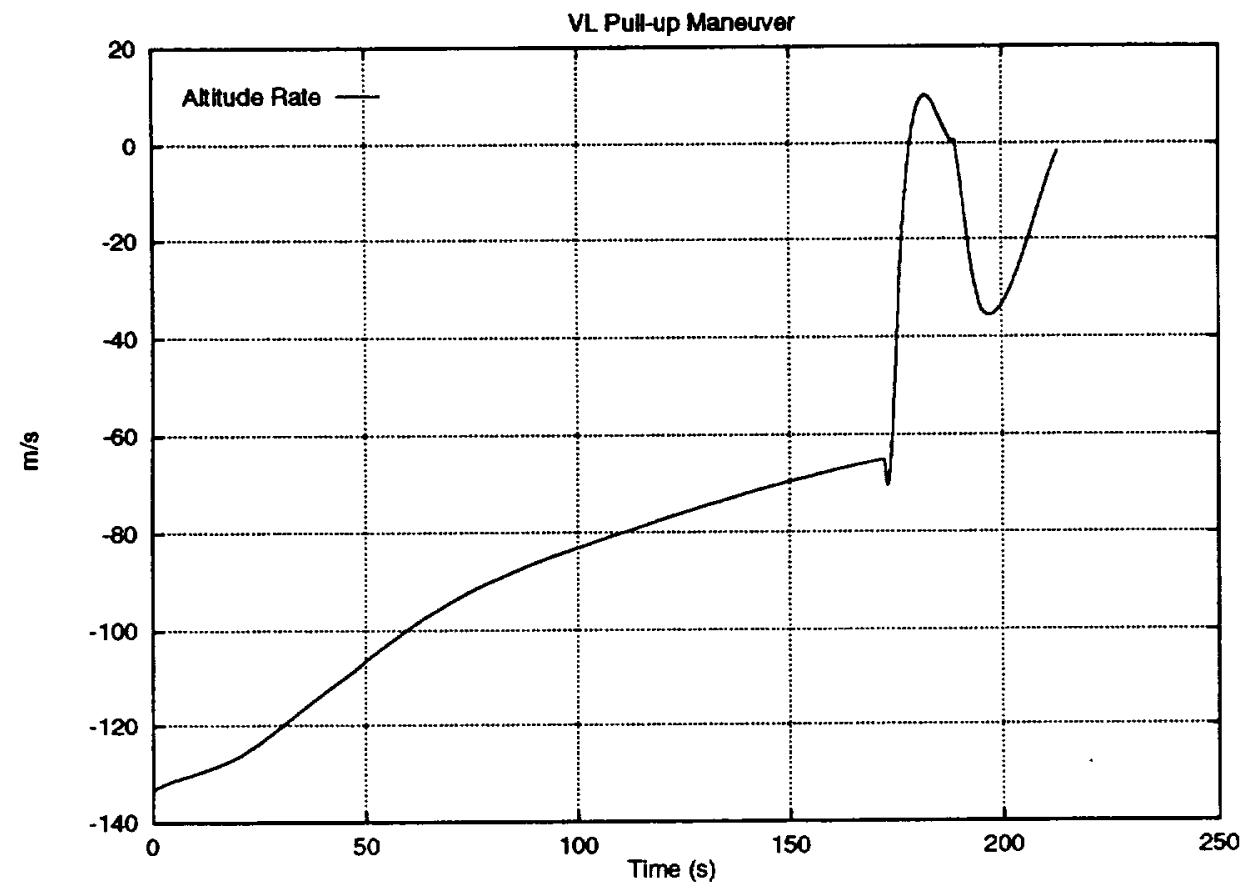

Figure 38. VL pullup maneuver, altitude rate versus time. 


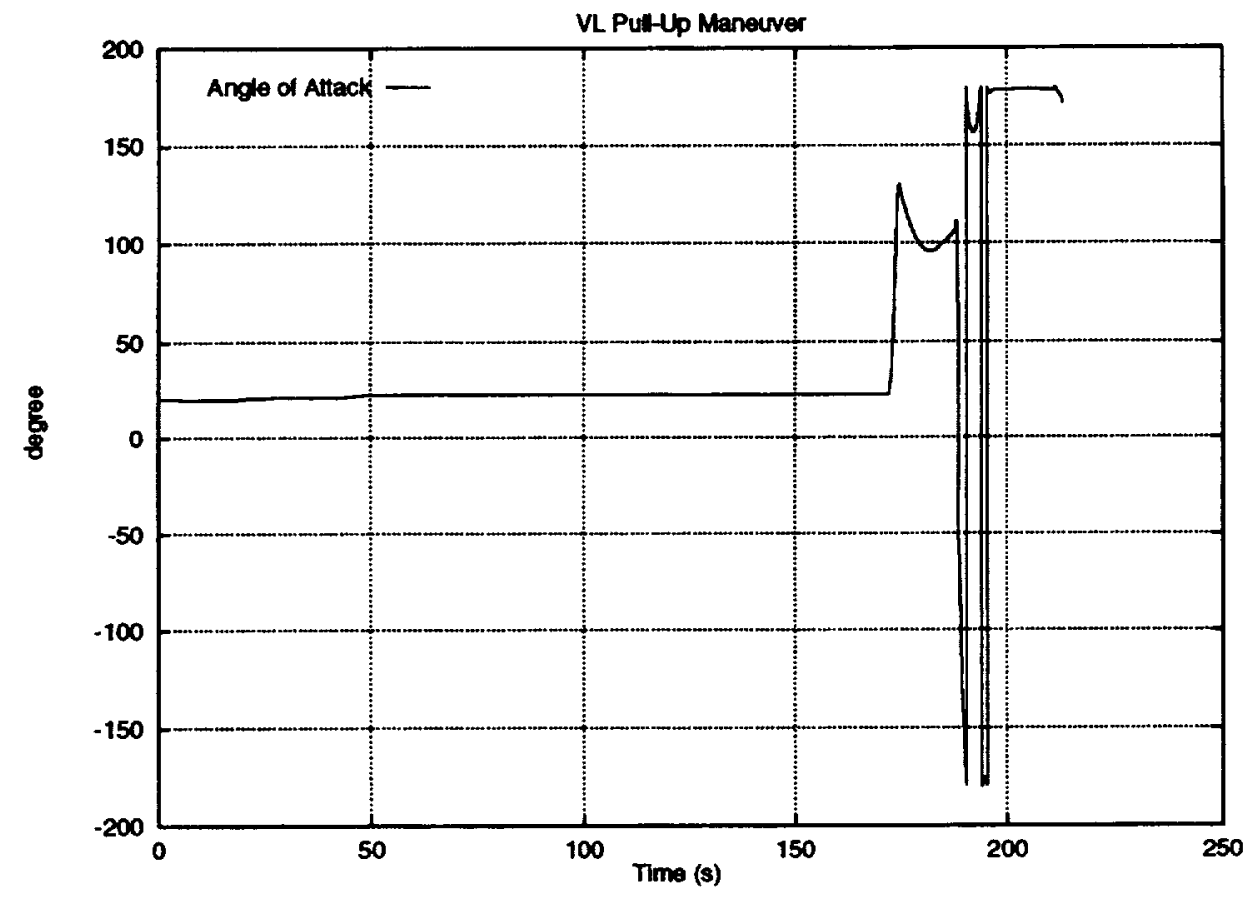

Figure 39. VL pullup maneuver, angle of attack versus time.

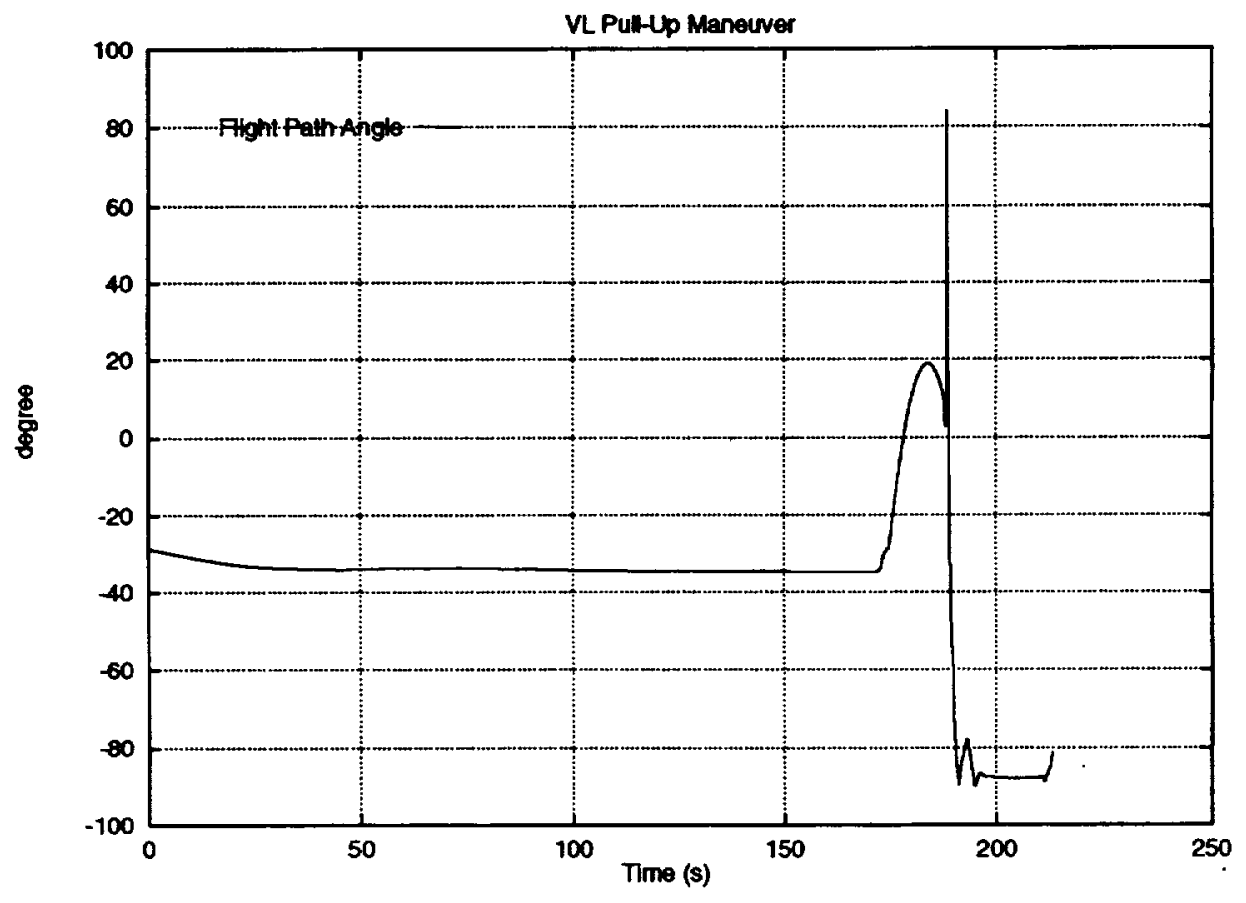

Figure 40. VL pullup maneuver, flight path angle versus time. 


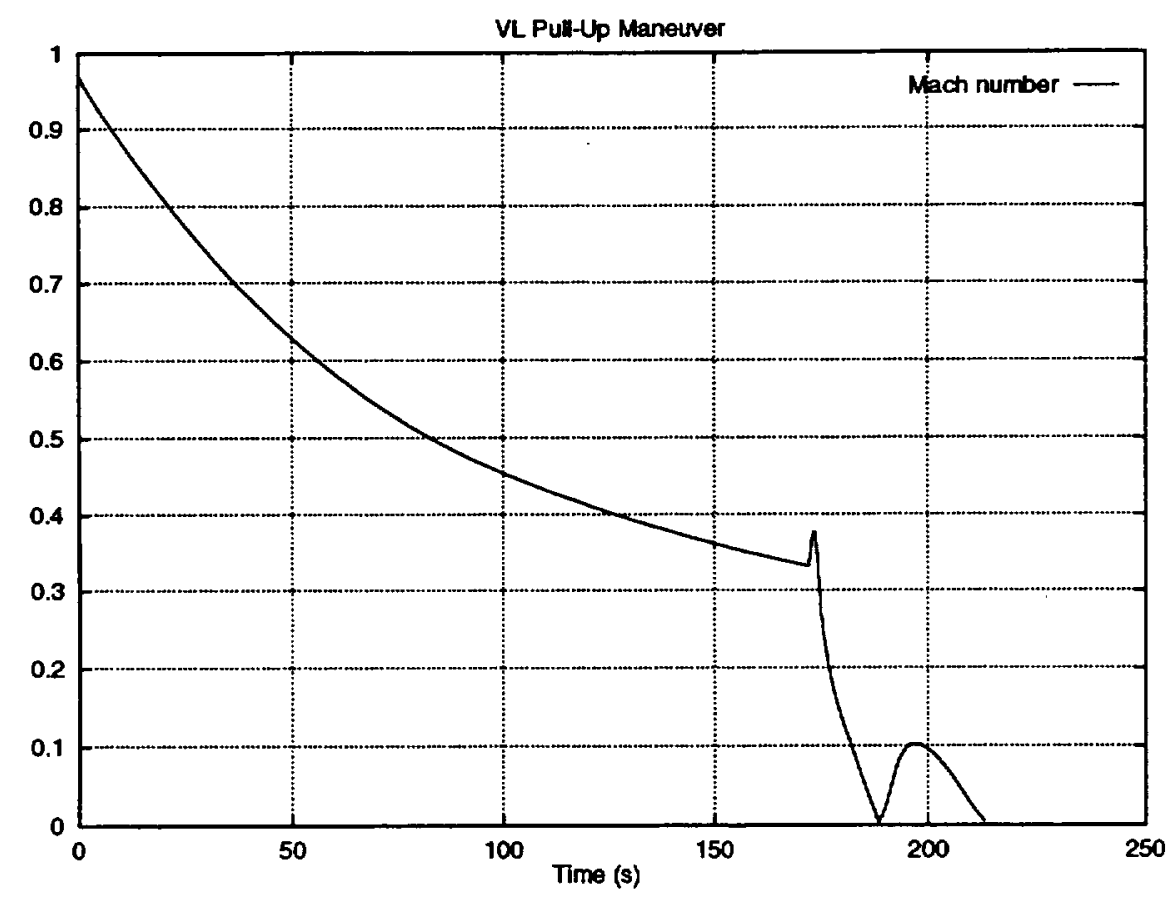

Figure 41. VL pullup maneuver, Mach number versus time.

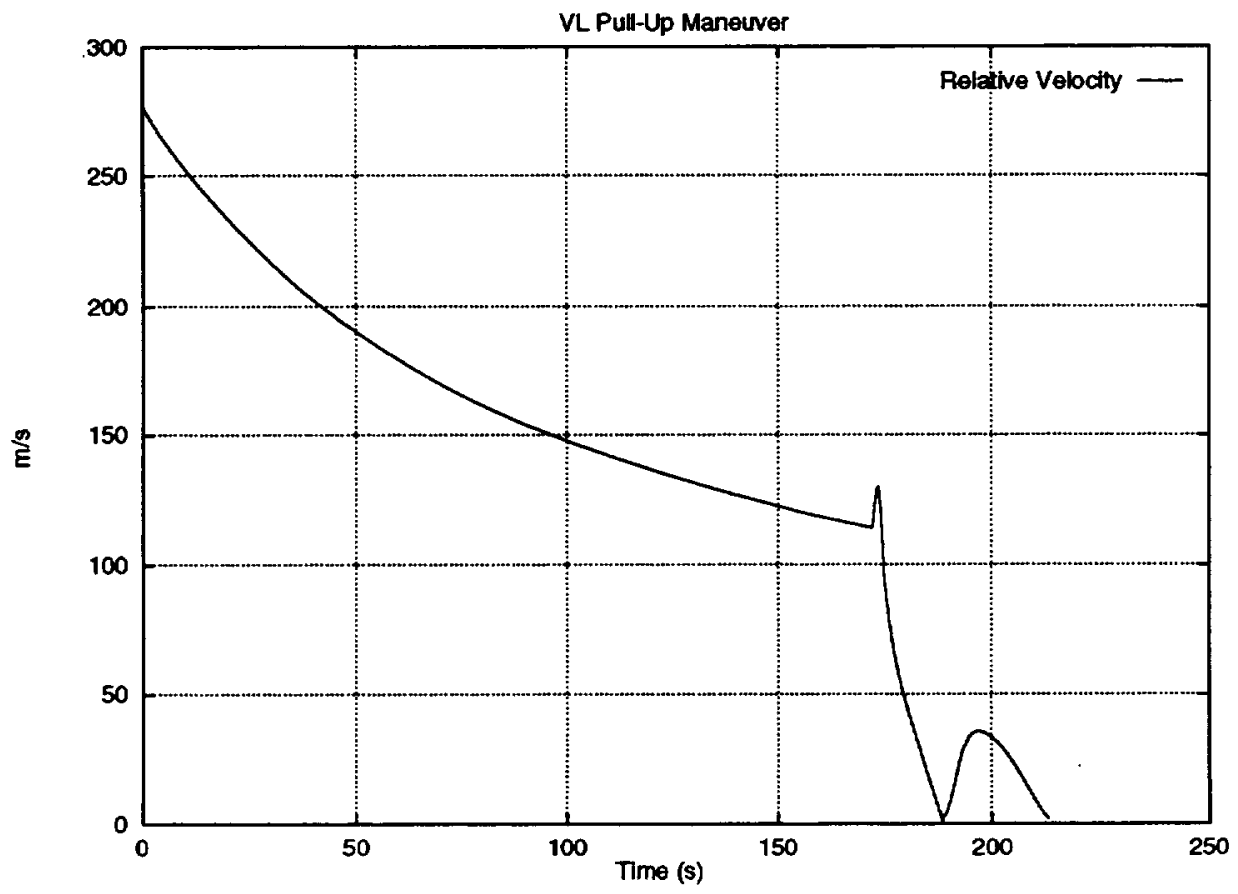

Figure 42 . VL pullup maneuver, relative velocity versus time. 


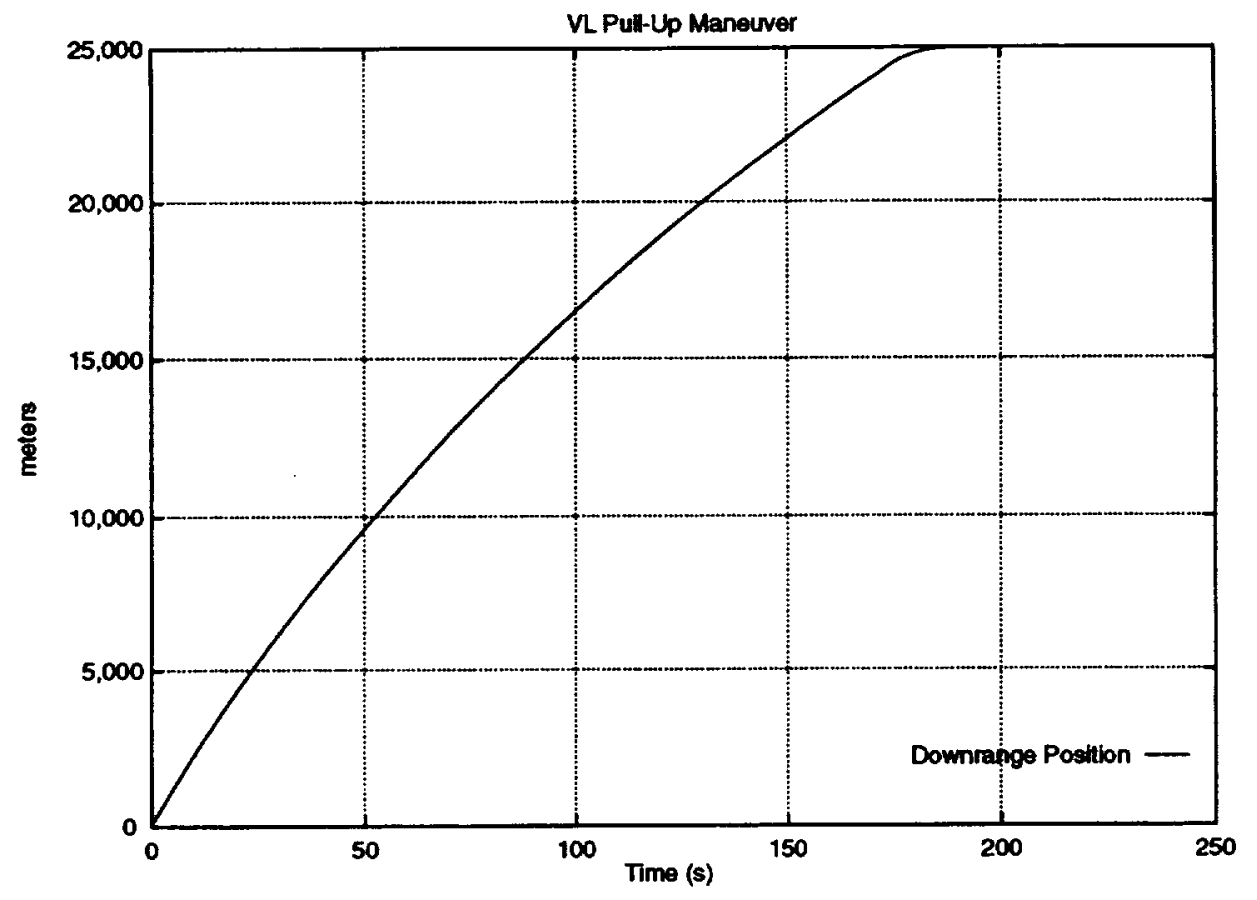

Figure 43. VL pullup maneuver, downrange position versus time.

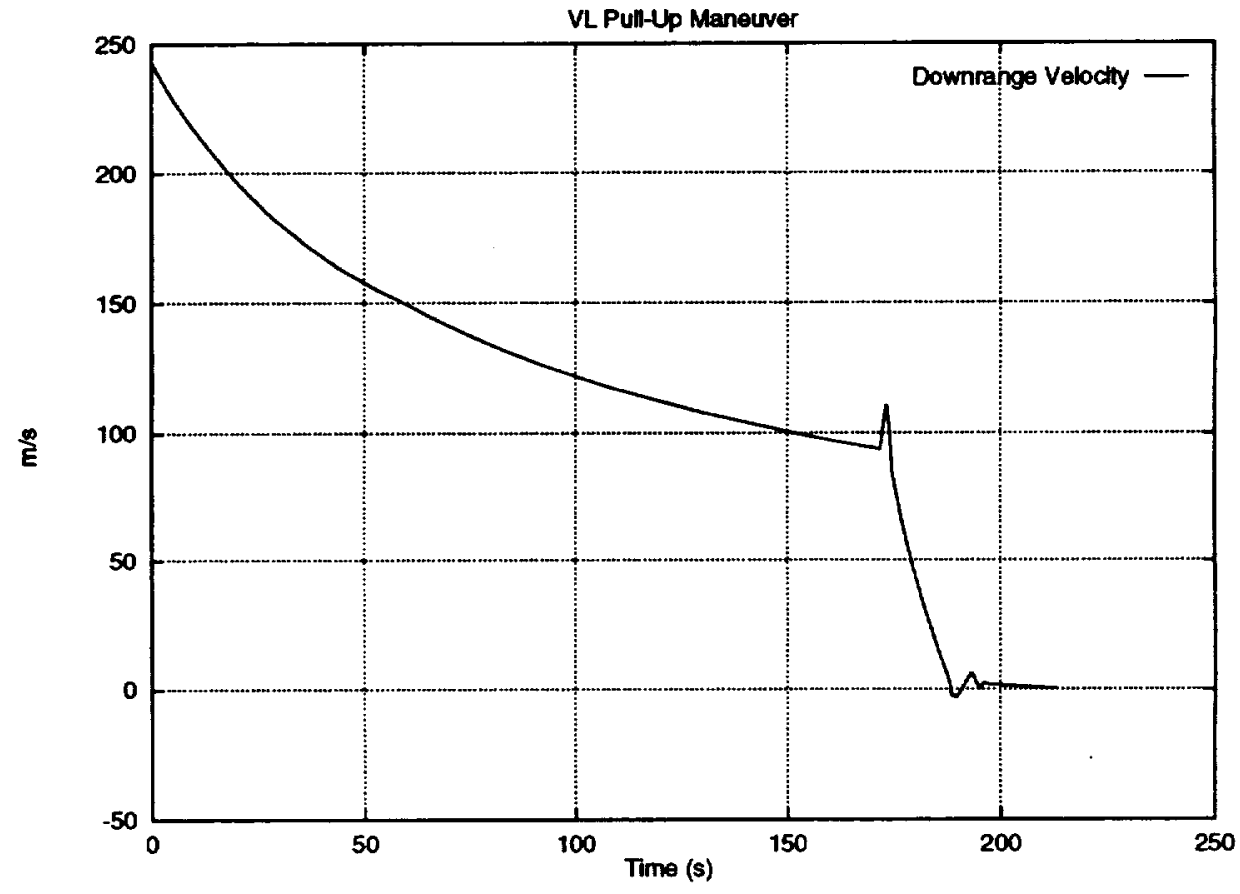

Figure 44. VL pullup maneuver, downrange velocity versus time. 


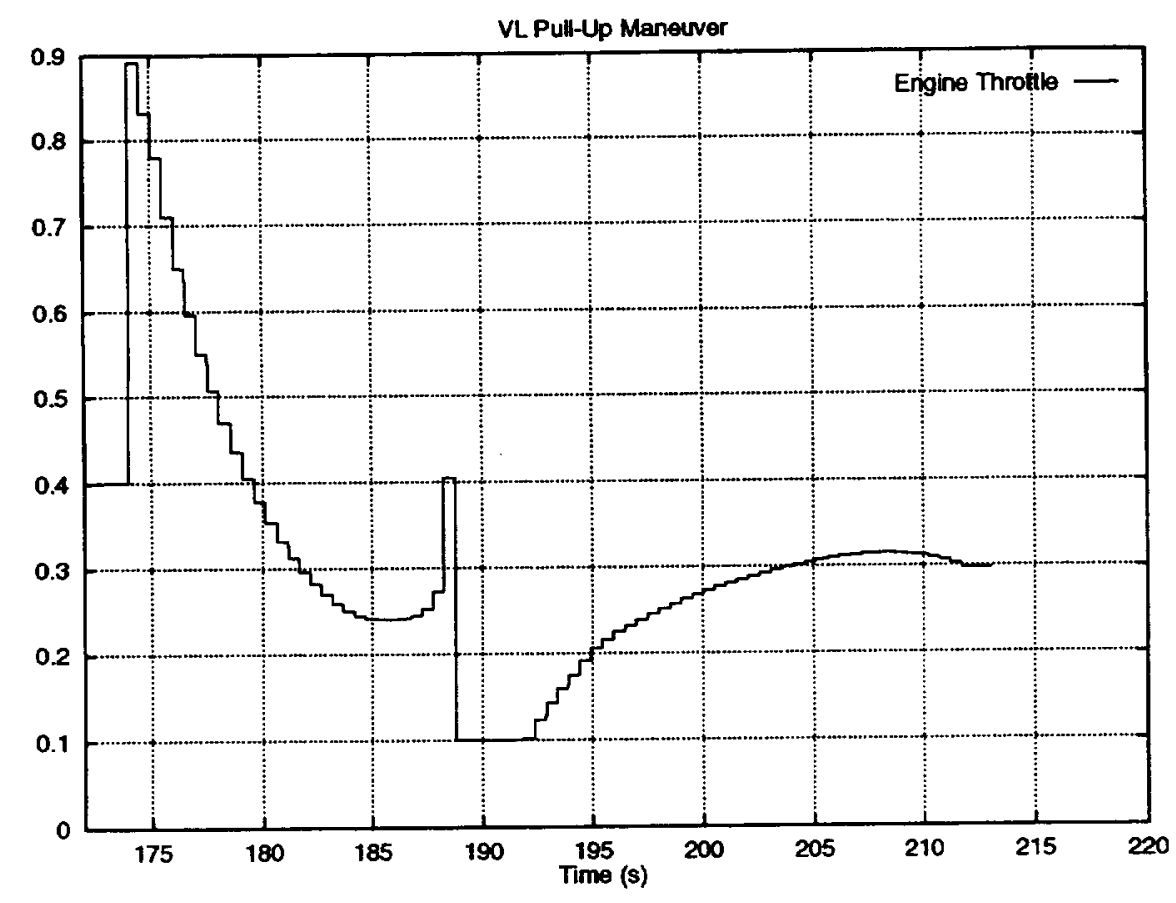

Figure 45. VL pullup maneuver, engine throttle versus time.

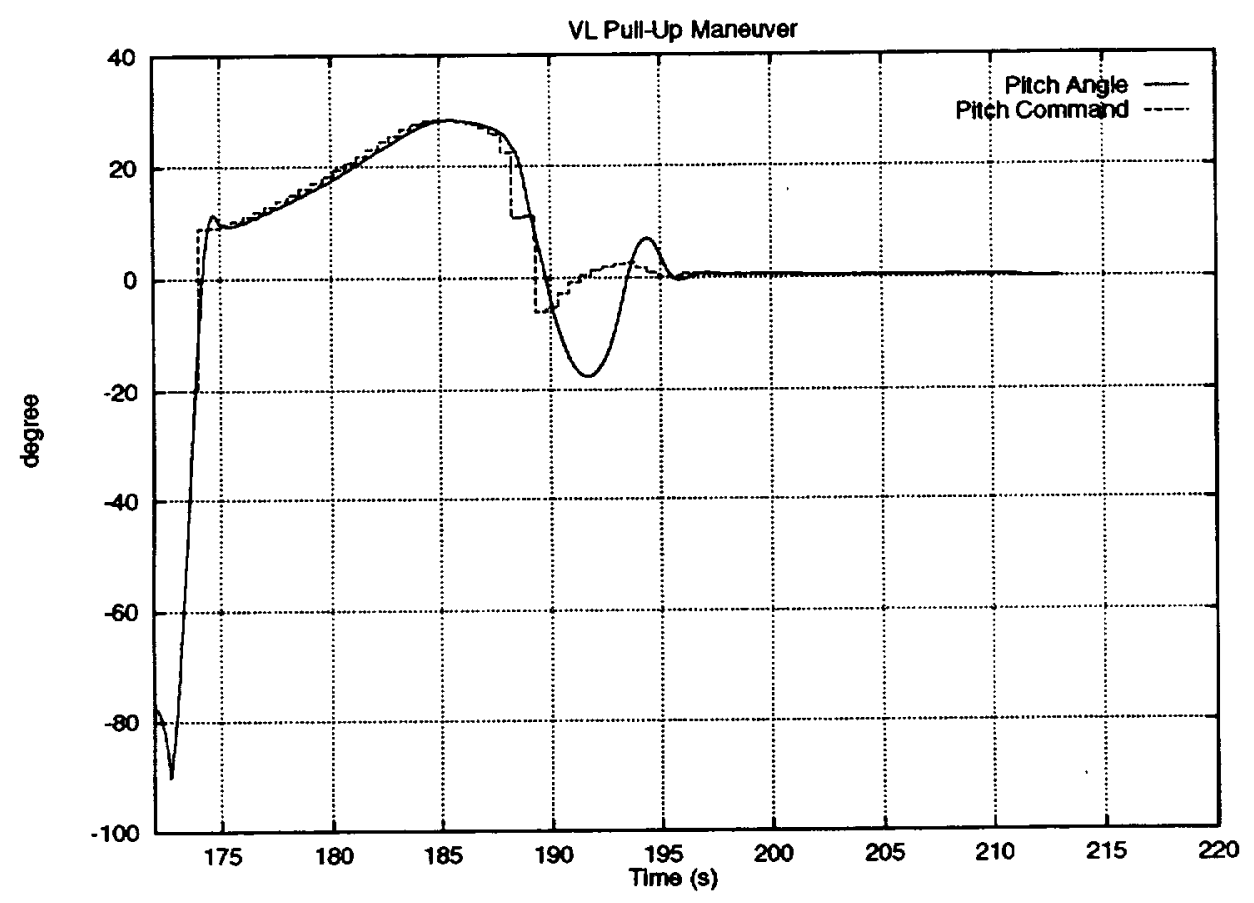

Figure 46. VL pullup maneuver, pitch angle and command versus time. 


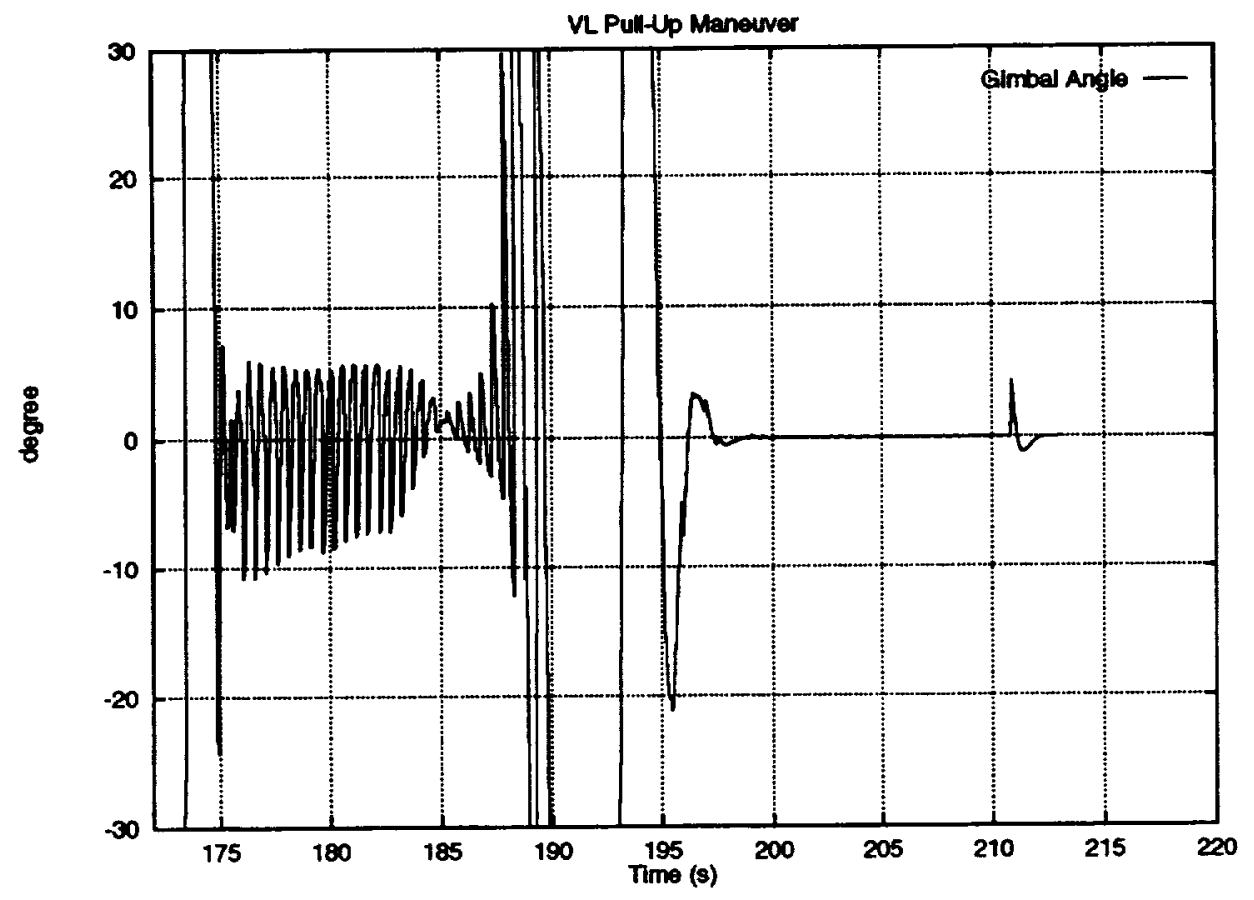

Figure 47. VL pullup maneuver, gimbal angle versus time.

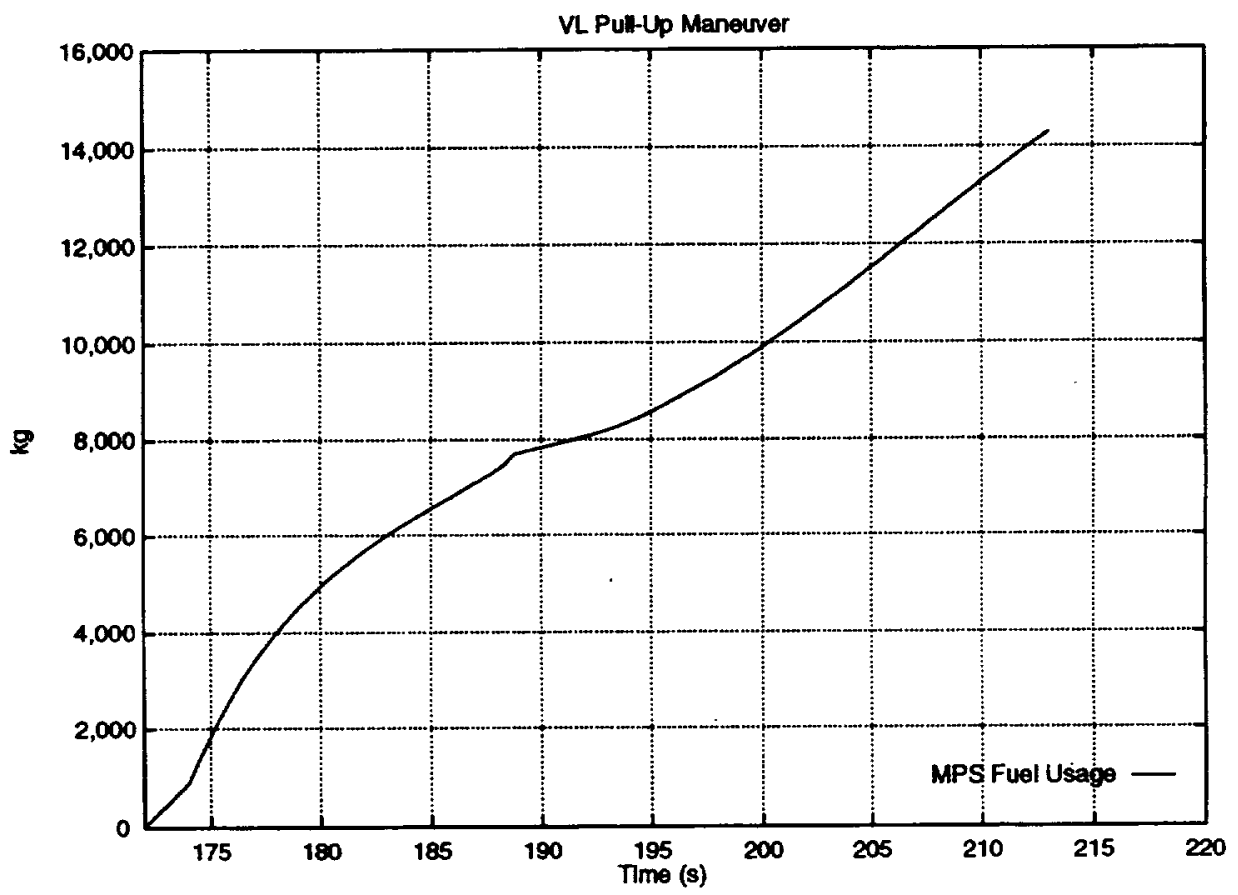

Figure 48. VL pullup maneuver, main propulsion system propellant usage versus time. 


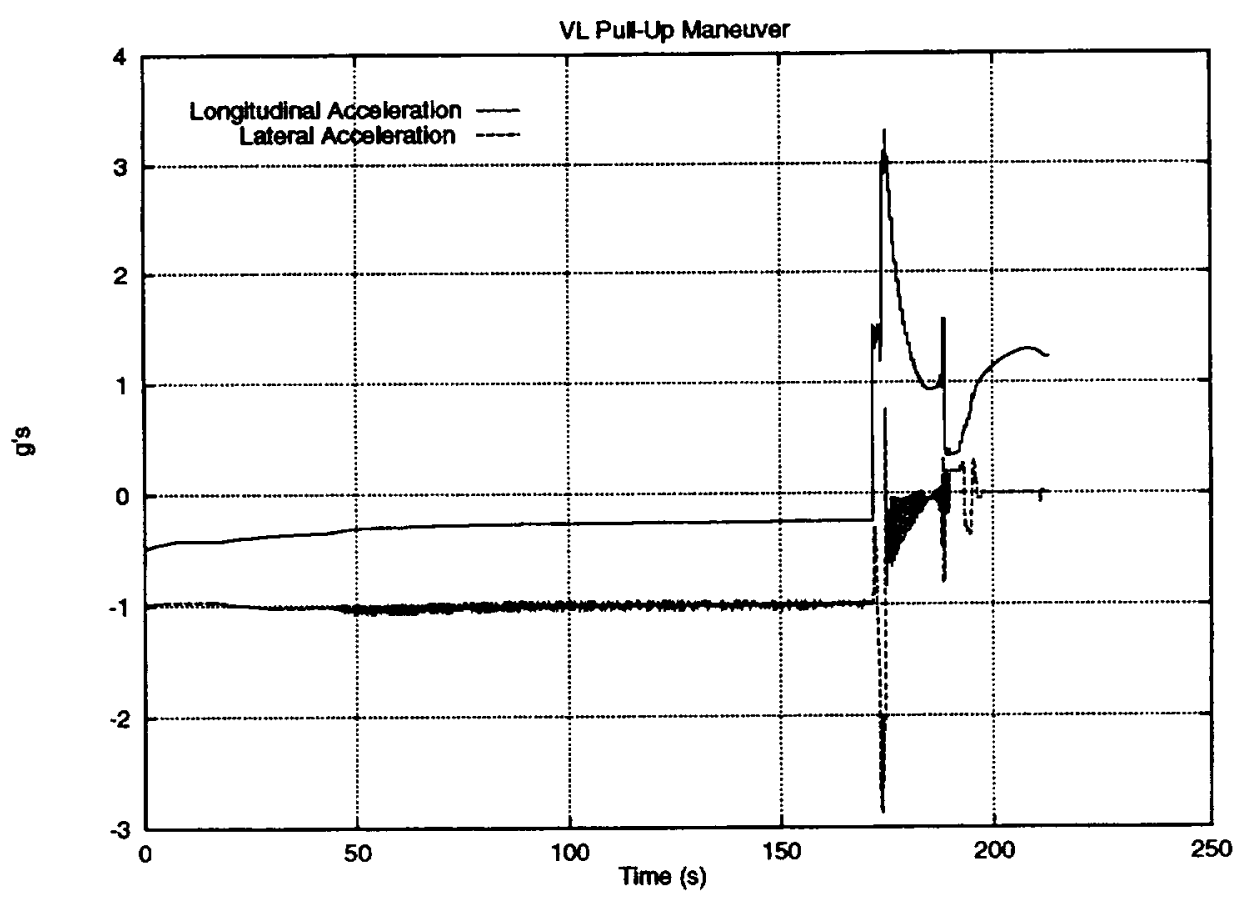

Figure 49. VL pullup maneuver, vehicle accelerations versus time.

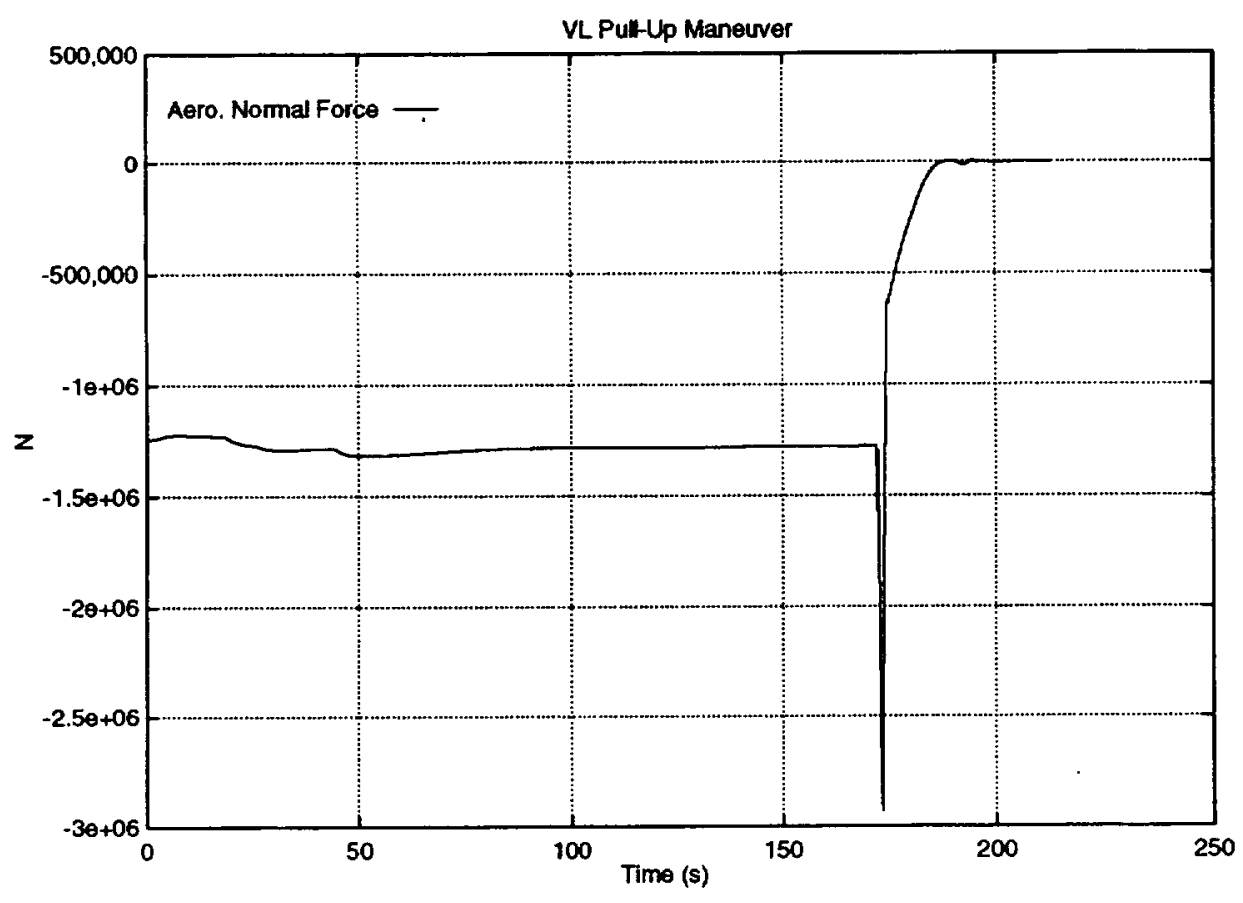

Figure 50. VL pullup maneuver, aerodynaic normal force versus time. 


\subsection{DISCUSSION}

From the G\&C analysis presented above, no landing option investigated can be declared infeasible or preclusive of future analysis. In each landing scheme, a successful touchdown was modeled within the propellant budget. The level of detail within this analysis did not reveal any stark infeasibilities within the three options.

The three landing options differ greatly because of the altitude at which the vehicle initiates the inversion. The higher inversion of the aerodynamic option results in much shorter downrange travel than the other options, since the vehicle flies a greater portion of its trajectory at a $180^{\circ}$ angle of attack, as described in section 5.2.1.3. The other two options call for a significant portion of flight at much lower angles of attack (a constant $20^{\circ}$ in these simulations; however, when fully designed, guidance would command a varying angle of attack as part of its energy management and targeting schemes). The aerodynamic lift will reduce the descent rate (as compared to the aerodynamic inversion option), while the drag decelerates the VL in the downrange direction. The increased time aloft results in increased downrange travel for these two options. As another consequence, the propulsive inversion and the powered pullup will require more time until touchdown. These differences will strongly influence guidance targeting schemes.

In addition, the engines, once ignited, provide thrust primarily to reduce descent rate. During the propulsive inversion and the powered pullup, the lit engines provide thrust while the vehicle flies at angles of attack below $90^{\circ}$. This thrust accelerates the vehicle in the downrange direction. Consequently, under the propulsive and pullup landing options, the VL must not only reduce its descent rate to nearly zero at landing, but, in addition, remove the downrange velocity imparted by the ignited engines during the respective inversion or pullup. Therefore, these two options will, in general, probably require that the $\mathrm{VL}$ consume more fuel than the aerodynamic option.

The nonoptimized and little-tuned G\&C algorithms used in these analyses provided successful simulated landings without dispersions or detailed modeling. This offers some confidence in the feasibility of designing adequate G\&C algorithms for the VL. The system will, however, impose major subsystem constraints and requirements and will require significant increases in sophistication in some areas.

Guidance will have to correct significant disturbances to flight path angle in a robust manner. The pullup option introduces significant disturbance, as seen in figure 51, where, in comparison, the propulsive maneuver experiences little change in flight path angle (the aerodynamic maneuver faces still less disturbances). It must also account for the required control torques in addition to descent rate when commanding throttle level. Low throttle reduces control margin, so guidance will need to consider attitude and attitude rate errors in order to balance the control requirements with the descent rate requirements. This implies a closely integrated G\&C system, which must share parameters and negotiate engine throttle levels. This closeness might reduce the overshoot seen in the propulsive and pullup simulations.

While each of the three simulated concepts touched down successfully, questions still remain about the controllability of this VL configuration. With the current configuration (aerodynamic shape and mass properties), the aerodynamic control surfaces are inadequate to control the vehicle's angle of attack during the reentry flight phases (especially subsonic flight). The vehicle's shape and/or the aerodynamic control surfaces will require redesign or resizing to correct this deficiency. These analyses also assumed that large control torques were available from either RCS or TVC systems to achieve the successful landings. Although the torque levels assumed were large, they were not 
completely unrealistic; however, the feasibility of implementing effectors to produce these large torques needs further investigation.

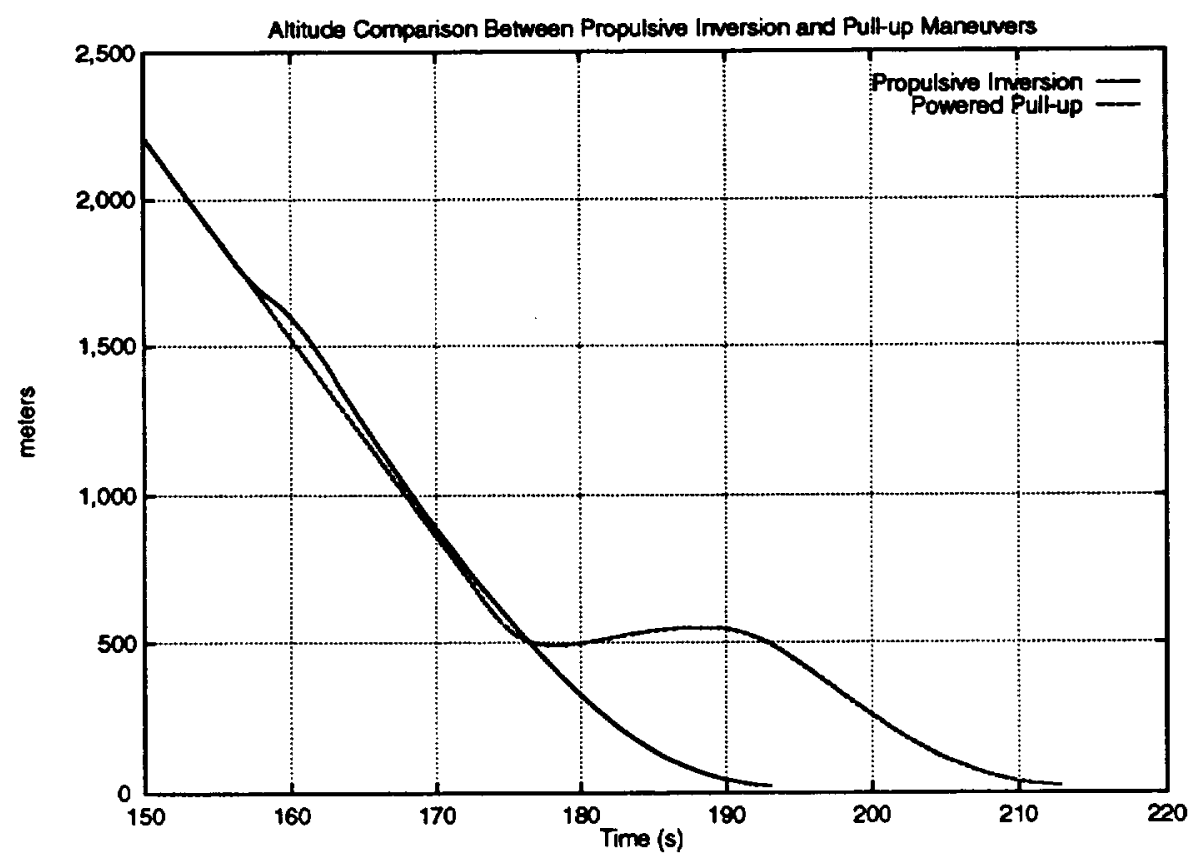

Figure 51. Altitude comparison between propulsive inversion and pullup maneuvers.

Due to the lack of lateral/directional aerodynamic coefficients, these analyses were limited to investigations in the pitch plane. While the roll and yaw axes should be controllable during the terminal descent and landing due to the capabilities of the gimballed engines, the controllability during the inversion and pullup maneuvers is questionable since it is unlikely that these maneuvers will be purely planar.

In simulating each of the concepts, a lack of detailed design data required the capabilities of the TVC actuators to be assumed. The actuators were modeled as second-order dynamic systems with a bandwidth and damping ratio selected on experience with the characteristics of actual TVC actuators. The actuator models did not limit the accelerations and rates, but did limit the gimbal angle to $30^{\circ}$. The $30^{\circ}$ gimbal angle range was required to obtain the control torque needed for the propulsive inversion and powered pullup maneuvers (this large gimbal requirement will impact the aft skirt design and the selection/design of the actuator and propellant feed system).

By allowing guidance to determine and control the engine throttle levels without regard to the control system, the effectiveness of the gimbaled engines to produce control torque was reduced as the engine throttle level was lowered to meet the trajectory constraints. The reduced thrust level of the main engines required larger gimbal angles to produce the needed torque to control the vehicle. Increased integration of the G\&C design and proper mission planning should reduce the maximum gimbal angle requirement.

The difference in acceleration profiles between the three options will strongly influence the design strategy of the engine propellant system. For the aerodynamic inversion option, the vehicle obtains a tail-first attitude prior to igniting the engines. Deceleration in this attitude would tend to seat the propellant masses in the aft ends of their respective tanks. The other two options require engine ignition at a nose-first vehicle attitude or during a pitching maneuver from a nose-first 
attitude. Propellant mass seating under these conditions may prove much more difficult to predict or to control.

Many of the problems associated with the vehicle controllability during the inversion could be avoided by scheduling the maneuver to take place in orbit, prior to atmospheric entry. The inversion could be performed in the absence of aerodynamic forces and moments with greater luxury and utilizing far lower control torques. Such an orbital inversion maneuver would likely exhibit the same relatively low fuel consumption seen in the aerodynamic inversion landing profile without the control difficulties encountered during the large angle-of-attack oscillations of the aerodynamic inversion maneuver.

The orbital inversion option was investigated and quickly deemed infeasible. The inversion of the vehicle prior to reentry would cause a ballistic-type flight path, during which the aft end of the VL would be subjected to extremely large aerodynamic heating. Also, the reentry flight would impose large aerodynamic loads on the engine cluster. It is unlikely that the engines could withstand these environments without the aid of a heat shield or similar heat dispersion system. Lastly, the poor aerodynamic characteristics of the VL in this attitude would constrain the lateral maneuvering capability of the vehicle during reentry. Consequently, deorbit targeting capabilities would be severely restricted, since most of the crossrange capability of the VL would be reserved for lateral maneuvering to correct for reentry dispersions such as winds and off-nominal deorbit burns.

\subsection{FUTURE WORK REQUIRED TO SHOW FEASIBILITY}

This section recommends future analysis that would be required in order to select a landing option. The analyses listed are fundamental to establishing the feasibility of guiding and controlling the landing phase of a VL design and should be of top priority in maturing the VL RLV from its current highly conceptual state to a preliminary design.

\subsection{6-DOF Targeting}

The landing analysis presented in this report assumes that the vehicle is in the proper inertial plane at the reentry/landing interface and remains in that plane through touchdown. Lack of lateral aerodynamic data forced the development of the guidance scheme, presented in section 3, to target a state in a two-axis coordinate system only. Targeting an out-of-plane state would have required a yaw rotation to an attitude for which sufficient aerodynamic effects could not be simulated. The current guidance scheme allows the targeting of vertical position, velocity, downrange velocity, and final attitude. Lateral targeting can be added without much difficulty, but will likely complicate the guidance static trim routine and the control system design.

\subsection{6-DOF Dispersion Analysis}

A complete 6-DOF landing dispersion analysis is needed to prove the feasibility of the VL concept. The dispersion analysis should, at a minimum, test the performance of the navigation and $\mathrm{G} \& \mathrm{C}$ systems to the following dispersions and uncertainties:

- Initial condition dispersions (resulting from reentry dispersion analysis)

- Thrust uncertainties (level, buildup, direction, throttle rate capability)

- Aerodynamic coefficient uncertainties 
- Mass properties dispersions

- Navigation errors

- Environment dispersions (density, pressure, temperature, winds).

The criteria upon which to judge the performance of the system should include:

- Propellant consumption

- Maximum gimbal angles

- Aerodynamic loads on vehicle

- Engine bell hinge moments

- Aerodynamic control surface hinge moments

- TVC actuator loads

- Acceleration profile (in relation to propellant feed system)

- Maximum accelerations on vehicle

- Touch down conditions

- Downrange position and velocity

- Vertical velocity

- Vehicle attitude

- Structural landing loads.

Such analysis should extend beyond the simulations described in this report to include complete landing dynamics and should provide Monte Carlo capability in order to statistically describe performance.

\subsection{Propulsion System}

Engine selection for a VL configuration presents unique design challenges. The engines, in addition to being restartable in flight after a possibly lengthy stay in orbit, must provide the proper thrust-to-weight (T/W) ratio required for both ascent and landing. The engines currently considered for the VL operate at a thrust capability that requires low throttling (around 20 to 30 percent) to achieve the necessary T/W for landing, assuming four engines. This problem could be alleviated (at the cost of significant increase in system complexity) by redesigning the propulsion concept to include four smaller engines, sized for landing and augmenting the thrust of larger engines during ascent. As discussed above, the throttle rate should be included in a 6-DOF dispersion analysis as this rate will drive the selection and/or design of the propulsion system.

\subsubsection{Engine-Out Analysis}

Assuming that the VL carries human passengers, the engine system will likely have the additional constraint of providing a safe landing in the event of one engine failure. An engine system with little throttle range capability will severely impact the design of the vertical landing trajectory when there exists the constraint of "engine-out capability." For instance, assume that the VL engines have a minimum throttle level of 70 percent and a nominal landing trajectory is designed utilizing two engines. Should one engine fail, the remaining engine cannot simply double its throttle level to compensate for the resulting thrust acceleration loss. 
Now, assume that the same minimum throttle level and a nominal landing trajectory has been designed utilizing three engines. In the event of an engine failure late in the landing flight, the remaining two engines still cannot throttle up enough to compensate for the thrust loss $(3 * 70$ percent $>2 * 100$ percent). With a 70-percent throttle level lower bound, four engines must be used for landing in order to provide an engine-out capability. The problem then exists of designing a landing trajectory using four engines at a minimum throttle level of 70 percent. This may not be an easy task considering the same engines are sized to provide the $\mathrm{T} / \mathrm{W}$ needed for ascent.

\subsection{Propellant Management}

Successful landing engine ignition will depend on reliable propellant seating. Because of the short landing times of 110 to $220 \mathrm{~s}$, there is little room for propellant feed error, making the propellant management design a critical area in need of analysis. A detailed propellant location and slosh analysis is required to properly design the propellant management system (tank baffles, propellant acquisition system, etc.) and the vehicle control system software and hardware.

Should the landing engines require cryogenic propellant, the issue of boil-off is a concern during the potentially long mission durations (approximately 10 days for an International Space Station resupply mission).

Current cryogenic propellant systems require a "chill-down" period prior to ignition. This is necessary to prevent system shock due to large temperature gradients. Should the landing engines require cryogenic propellant, the integration of this chill-down period into the flight design would be necessary. Safety considerations will likely require the chill-down period to begin after the high aerodynamic heating region of reentry. The current VL reentry trajectory provides approximately 14 $\mathrm{min}$ between exiting the high heating region and the reentry/landing interface at $16,800 \mathrm{~m}$. If engine chill-down cannot be accomplished within this time period, the landing flight design options will suffer further constraints.

\subsection{Sensor Dynamics}

The onboard navigation system must provide accurate position, attitude, and acceleration data to the G\&C subsystems during the entire landing phase of flight. The position navigational sensors (radar altimeter, GPS antenna, etc.) must be located on the vehicle to assure no loss-of-signal during and after the inversion maneuver while in the presence of high acceleration disturbances. Input from navigational software analysis and simulation would be required for navigational dispersion values needed in the 6-DOF dispersion analysis.

\subsection{Loads}

Since the vehicle flies at significant angles of attack in regions of high dynamic pressure during the inversion and landing phases in all three concepts studied, the aerodynamic loads imparted to the vehicle should be analyzed and accommodated by the vehicle's structural design. The aerodynamic inversion concept imparts unusual aerodynamic loads through the large angle-of-attack oscillations experienced while stabilizing the vehicle to its tail-first attitude. While some undispersed estimates of these aerodynamic loads (included in section 5) may be used to begin a study of structural impacts, they are not sufficiently detailed for use in structural design choices.

Each of the landing concepts, by definition, requires the vehicle to fly in a tail-first attitude before touchdown. The effects of the aerodynamic loads on flight hardware not accustomed to 
experiencing aerodynamic loads (such as the engine bells, the OMS and/or RCS engines, heat shields, etc.) is an important issue that must be addressed before any of the concepts could be considered feasible. Not only must the hardware be sufficiently strong to withstand the aerodynamic loads, but it must also be capable of performing its function in this environment. An example of this concern is the interaction between the engine bells and the TVC actuators. The engine bells must withstand both the aerodynamic loads imparted by air flow into the bell and the aerodynamic loads associated with the vehicle's angle of attack. These loads will additionally create hinge moments (moments about the engine gimbal) which will affect both the gimbal structural design and the TVC actuators. The TVC actuators must be designed (structural integrity, sizing, power requirements, etc.) to gimbal the engines during powered flight against the opposition of the large loads imparted by the hinge moments.

Another interaction between the G\&C and structural designers is the assessment of the vehicle's capability to withstand landing loads. As currently envisioned, the VL vehicle will land on four landing legs. These legs must withstand the loads associated not only with the weight of the vehicle, but also with the loads associated with vertical and horizontal touchdown velocity and nonvertical attitudes at touchdown. The structural capability of the landing legs and airframe will probably determine the limits of the G\&C touchdown targets, and these targets will be regularly updated as the vehicle design matures.

\subsection{Aerodynamics}

As previously mentioned, the immaturity of the VL configuration resulted in the availability of only the longitudinal aerodynamic coefficients for controllability and performance assessments.

Lateral and directional aerodynamics are required by future studies to prove the feasibility (or show infeasibility) of any of the proposed landing concepts, since it is unlikely the vehicle will experience exclusively planar aerodynamic maneuvers.

The longitudinal characteristics of the vehicle were shown in these analyses to be marginal in terms of stability and controllability. However, this situation could be improved through modifying the configuration or resizing aerodynamic control surfaces. Another option may be to include additional control surfaces such as movable fins, canards, speed brakes, or aerodynamic control surfaces located at the nose.

The vehicle's aerodynamic characteristics will change in the immediate vicinity of the landing site. These changes may result in forces and moments which are dramatically different from those during the more extensive period of atmospheric flight. Due to the short period during which these effects are present, the G\&C system will have a limited opportunity to identify and accommodate disturbances and variations to them. These ground effects must be carefully analyzed and accounted for in the modeling of the VL landing in order to design a G\&C system that is satisfactorily robust during this critical mission phase. 


\section{REFERENCES}

1. Springer, A.: "Initial Aerodynamic Database for the Vertical Lander Reusable Launch Vehicle Configuration; VL001 8-1 Dataset.” MSFC memorandum ED34-01-95.

2 Cherry, G.W.: “A General, Explicit, Optimizing Guidance Law for Rocket-Propelled Spaceflight." AIAA, 1964. 


\title{
APPROVAL
}

\section{A GUIDANCE AND CONTROL ASSESSMENT OF THREE VERTICAL LANDING OPTIONS FOR RLV}

\author{
By M. Gallaher, D. Coughlin, and D. Krupp
}

The information in this report has been reviewed for technical content. Review of any information concerning Department of Defense or nuclear energy activities or programs has been made by the MSFC Security Classification Officer. This report, in its entirety, has been determined to be unclassified.

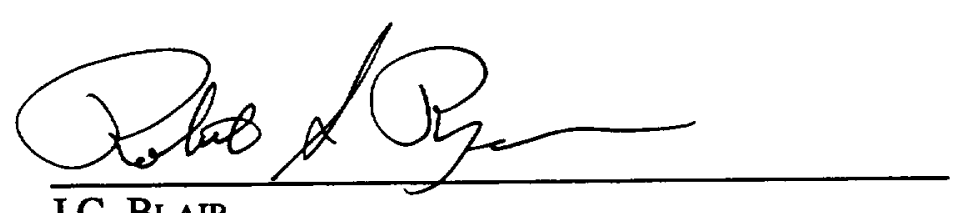

J.C. BLAIR

Director, Structures and Dynamics Laboratory 


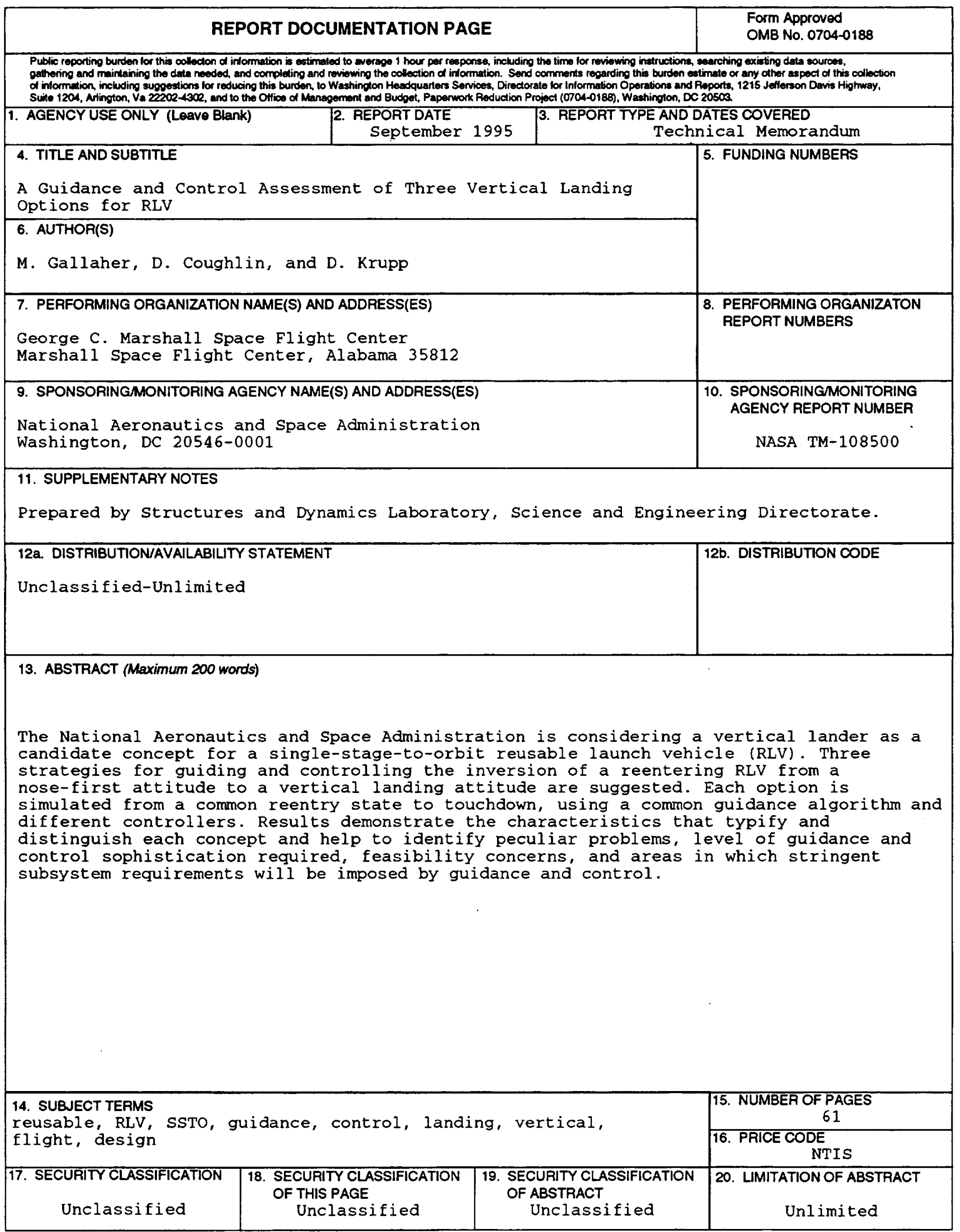

